Supporting Information

\title{
Site-selective $\alpha$-Alkoxyl Alkynation of Alkyl Esters Mediated by Boryl Radicals
}

\author{
Ao Guo ${ }^{\dagger}$ Jia-Bin Han, ${ }^{\dagger}$ Lei Zhu, ${ }^{\dagger}$ Yin Wei ${ }^{\S}$ and Xiang-Ying Tang ${ }^{\dagger *}$ \\ ${ }^{\dagger}$ School of Chemistry and Chemical Engineering and Hubei Key Laboratory of \\ Bioinorganic Chemistry and Materia Medica, Huazhong University of Science and \\ Technology, 1037 Luoyu Road, Wuhan 430074, People’s Republic of China \\ ${ }^{\ddagger}$ College of Chemistry and Materials Science, Hubei Engineering University, Hubei, \\ 432000, People's Republic of China. \\ ${ }^{\S}$ State Key Laboratory of Organometallic Chemistry, Shanghai Institute of Organic \\ Chemistry, Chinese Academy of Sciences, 345 Lingling Road, Shanghai 200032 China. \\ xytang@hust.edu.cn
}

Content

$\begin{array}{ll}\text { 1. General remarks } & \mathrm{S} 2\end{array}$

2. General Procedure for the Synthesis of 2a S3

3. General procedure for the Synthesis of $1 \mathrm{mmol}$ scale of $\mathbf{2 a} \quad \mathrm{S} 3$

4. General Procedure for the Synthesis of 3a S3

5. General Procedure for the Control experiments $\quad$ S4

6. General Procedure for the Derivatizations of Product $\mathbf{3 m}$. S5

$\begin{array}{ll}\text { 7. Spectroscopic data } & \text { S6-S59 }\end{array}$ 
General Remarks: ${ }^{1} \mathrm{H}$ NMR and ${ }^{13} \mathrm{C}$ NMR spectra were recorded on an Agilent DD2 400-MR spectrometer in $\mathrm{CDCl}_{3}$ with tetramethylsilane (TMS) as the internal standard; Chemical shifts $(\delta)$ are expressed in ppm and $J$-values are in Hz. Mass spectra were recorded with a HP-5989 instrument. Infrared spectra were recorded on a Perkin-Elmer PE-983 spectrometer with absorption in $\mathrm{cm}^{-1}$. The solvents were purchased and used as reveived. All reactions were monitored by TLC with Huanghai GF254 silica gel coated plates. Flash column chromatography was carried out using 300-400 mesh silica gel at increased pressure. 


\section{General procedure for the synthesis of 2}<smiles></smiles>

A $10 \mathrm{~mL}$ reaction tube equipped with a magnetic stir bar was charged with 4cyanopyridine (30 mg, $0.3 \mathrm{mmol}$ ), 4-cyanopyridine-1-oxide (72 mg, $0.6 \mathrm{mmol}$ ), Bis(neopentyl glycolato)diboron (135 mg, $0.6 \mathrm{mmol}), 1(0.3 \mathrm{mmol})$, and ethyl acetate $(3 \mathrm{~mL})$. The reaction mixture was stirred at $30^{\circ} \mathrm{C}$ for $10 \mathrm{~min}$. After completion (checked by TLC), the mixture was concentrated under reduced pressure and the crude product was purified by silica gel column chromatography (PE:EA $=40: 1)$ to give the corresponding products.

\section{General procedure for the synthesis of $2 \mathrm{a}$ in $1 \mathrm{mmol}$ scale}

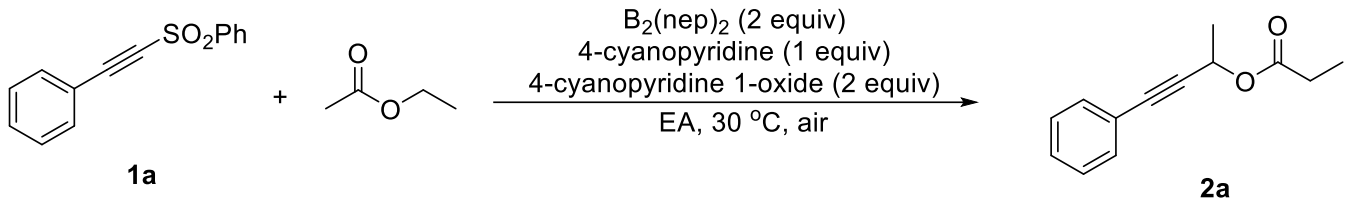

A $25 \mathrm{~mL}$ reaction tube equipped with a magnetic stir bar was charged with 4cyanopyridine (102 mg, $1.0 \mathrm{mmol})$, 4-cyanopyridine-1-oxide (240 mg, $2.0 \mathrm{mmol}$ ), Bis(neopentyl glycolato)diboron (452 mg, $2 \mathrm{mmol}$ ), [(phenylethynyl)sulfonyl]benzene (242 $\mathrm{mg}, 1 \mathrm{mmol})$, and ethyl acetate $(\mathrm{EA})(10 \mathrm{~mL})$. The reaction mixture was stirred at $30{ }^{\circ} \mathrm{C}$ for $10 \mathrm{~min}$. After completion (monitored by TLC), the mixture was concentrated under reduced pressure and the crude product was purified by silica gel column chromatography (PE:EA $=40: 1$ ) to afford (1-ethoxyethyl)phenanthridine 2a. Yield: $129 \mathrm{mg}, 68 \%$.

\section{General procedure for the synthesis of 3}

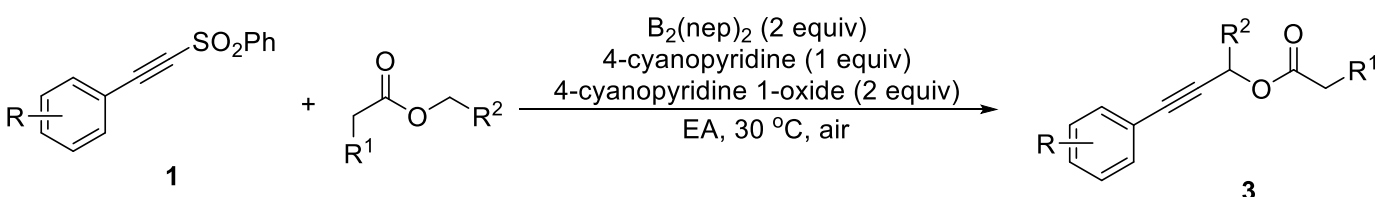


A $10 \mathrm{~mL}$ reaction tube equipped with a magnetic stir bar was charged with 4cyanopyridine (30 mg, $0.3 \mathrm{mmol}$ ), 4-cyanopyridine-N-oxide (72 mg, $0.6 \mathrm{mmol}$ ), Bis(neopentyl glycolato)diboron (135 $\mathrm{mg}, 0.6 \mathrm{mmol}), \mathbf{1}(0.3 \mathrm{mmol})$ and methyl acetate (MA) (3 mL). The reaction mixture was stirred at $30{ }^{\circ} \mathrm{C}$ for $30 \mathrm{~min}$. After completion (checked by TLC), the mixture was concentrated under reduced pressure and the crude product was purified by silica gel column chromatography $(\mathrm{PE}: \mathrm{EA}=40: 1)$ to give the corresponding products.

\section{General procedure of the control experiments}

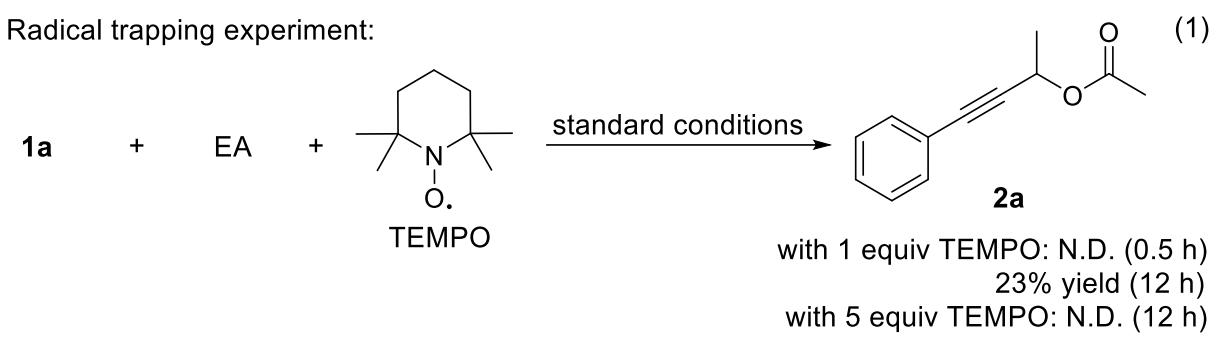

A $10 \mathrm{~mL}$ reaction tube equipped with a magnetic stir bar was charged with $\mathbf{1 a}(48 \mathrm{mg}$, $0.2 \mathrm{mmol}$ ), 4-cyanopyridine (20 mg, $0.2 \mathrm{mmol}$ ), 4-cyanopyridine-1-oxide (48 mg, 0.4 mmol), Bis(neopentyl glycolato)diboron (90 mg, $0.4 \mathrm{mmol}$ ), TEMPO (32 mg, 0.2 mmol or $156 \mathrm{mg}, 1.0 \mathrm{mmol})$, and ethyl acetate $(2 \mathrm{~mL})$. The reaction mixture was stirred at $30{ }^{\circ} \mathrm{C}$. The reaction was monitored by TLC. After indicated time, the reaction mixture was concentrated and purified by column chromatography if product was detected by TLC.

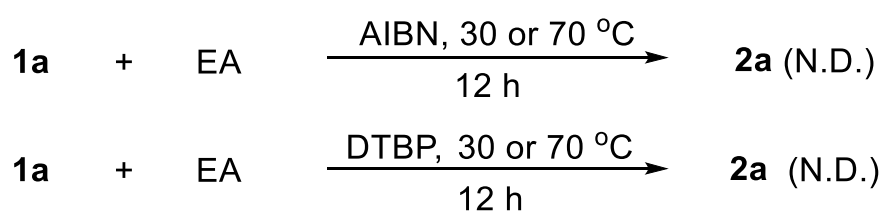

A $10 \mathrm{~mL}$ reaction tube equipped with a magnetic stir bar was charged with $1 \mathbf{a}(48 \mathrm{mg}$, $0.2 \mathrm{mmol}$ ), azobisisobutyronitrile (AIBN) (49 mg, $0.3 \mathrm{mmol}$ ) or Di-tert-butyl peroxide (DTBP, $44 \mathrm{mg}, 0.3 \mathrm{mmol}$ ), and ethyl acetate $(2 \mathrm{~mL})$. The reaction mixture was stirred at 30 or $70{ }^{\circ} \mathrm{C}$. After $12 \mathrm{~h}, \mathbf{2 a}$ was not detected by TLC analysis.

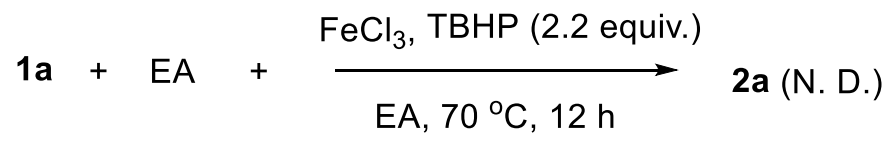


A $10 \mathrm{~mL}$ reaction tube equipped with a magnetic stir bar was charged with $\mathbf{1 a}(48 \mathrm{mg}$, $0.2 \mathrm{mmol}$ ), tert-butyl hydroperoxide (TBHP, $40 \mathrm{mg}, 0.44 \mathrm{mmol})$ ferric chloride $\left(\mathrm{FeCl}_{2}\right.$, $5 \mathrm{mg}, 0.04 \mathrm{mmol})$ and ethyl acetate $(2 \mathrm{~mL})$. The reaction mixture was stirred at $70{ }^{\circ} \mathrm{C}$. After 12 h, 2a was not detected by TLC analysis.

\section{Experiment procedure for the derivatizations of $3 \mathrm{~m}$.}

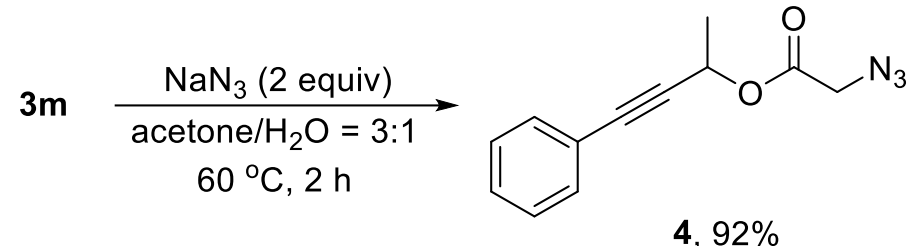

A $10 \mathrm{~mL}$ reaction tube equipped with a magnetic stir bar was charged with 4-phenylbut3-yn-2-yl 2-chloroacetate (3m, $0.15 \mathrm{mmol}, 33 \mathrm{mg})$, sodium azide $\left(\mathrm{NaN}_{3}, 19 \mathrm{mg}, 0.3\right.$ $\mathrm{mmol}), \mathrm{H}_{2} \mathrm{O}(500 \mu \mathrm{L})$, and acetone $(1.5 \mathrm{~mL})$. The reaction mixture was stirred at $60{ }^{\circ} \mathrm{C}$ for $2 \mathrm{~h}$. After completion (checked by TLC), the mixture was concentrated under reduced pressure and the crude product was purified by silica gel column chromatography $(\mathrm{PE}: \mathrm{EA}=40: 1)$ to afford 4-phenylbut-3-yn-2-yl 2-azidoacetate 4. Yield: $32 \mathrm{mg}, 92 \%$. Colorless oil.
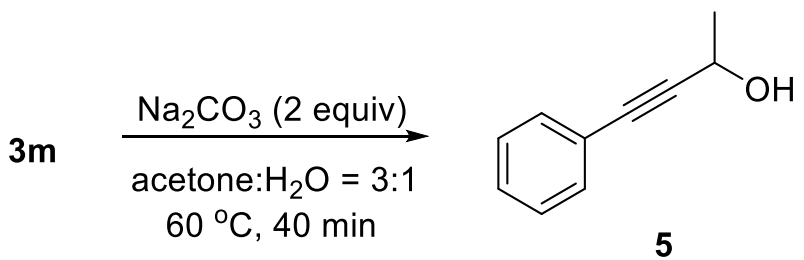

A $10 \mathrm{~mL}$ reaction tube equipped with a magnetic stir bar was charged with 4-phenylbut3-yn-2-yl 2-chloroacetate $(\mathbf{3 m}, 0.15 \mathrm{mmol}, 33 \mathrm{mg})$, sodium carbonate $\left(\mathrm{NaN}_{3}, 32 \mathrm{mg}\right.$, $0.3 \mathrm{mmol}), \mathrm{H}_{2} \mathrm{O}(500 \mu \mathrm{L})$, and acetone $(1.5 \mathrm{~mL})$. The reaction mixture was stirred at $60{ }^{\circ} \mathrm{C}$ for $40 \mathrm{~min}$. After completion (checked by TLC), the mixture was concentrated under reduced pressure and the crude product was purified by silica gel column chromatography (PE:EA = 10:1) to afford 4-phenylbut-3-yn-2-ol 5. Yield: $19 \mathrm{mg}, 86 \%$. Colorless oil.

\section{Spectroscopic data}




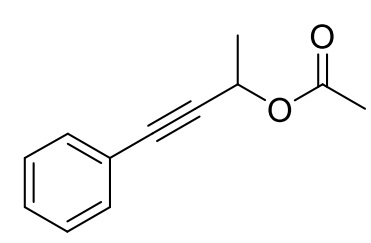

4-Phenylbut-3-yn-2-yl acetate (2a). A known compound ${ }^{[1]}$. Yield: 39 mg, 71\%. Colorless oil. ${ }^{\mathbf{1}} \mathbf{H}$ NMR $\left(400 \mathrm{MHz}, \mathrm{CDCl}_{3}\right)$ $\delta$ 7.46-7.43 (m, 2H), 7.32-7.29 (m, 3H), 5.69 (q, $J=6.4 \mathrm{~Hz}, 1 \mathrm{H})$, $2.11(\mathrm{~s}, 3 \mathrm{H}), 1.58(\mathrm{~d}, J=6.8 \mathrm{~Hz}, 3 \mathrm{H}) ;{ }^{13} \mathbf{C}$ NMR $(101 \mathrm{MHz}$, $\left.\mathrm{CDCl}_{3}\right) \delta 169.9,131.8,128.5,128.2,122.2,87.4,84.5,60.8,21.5,21.1$.

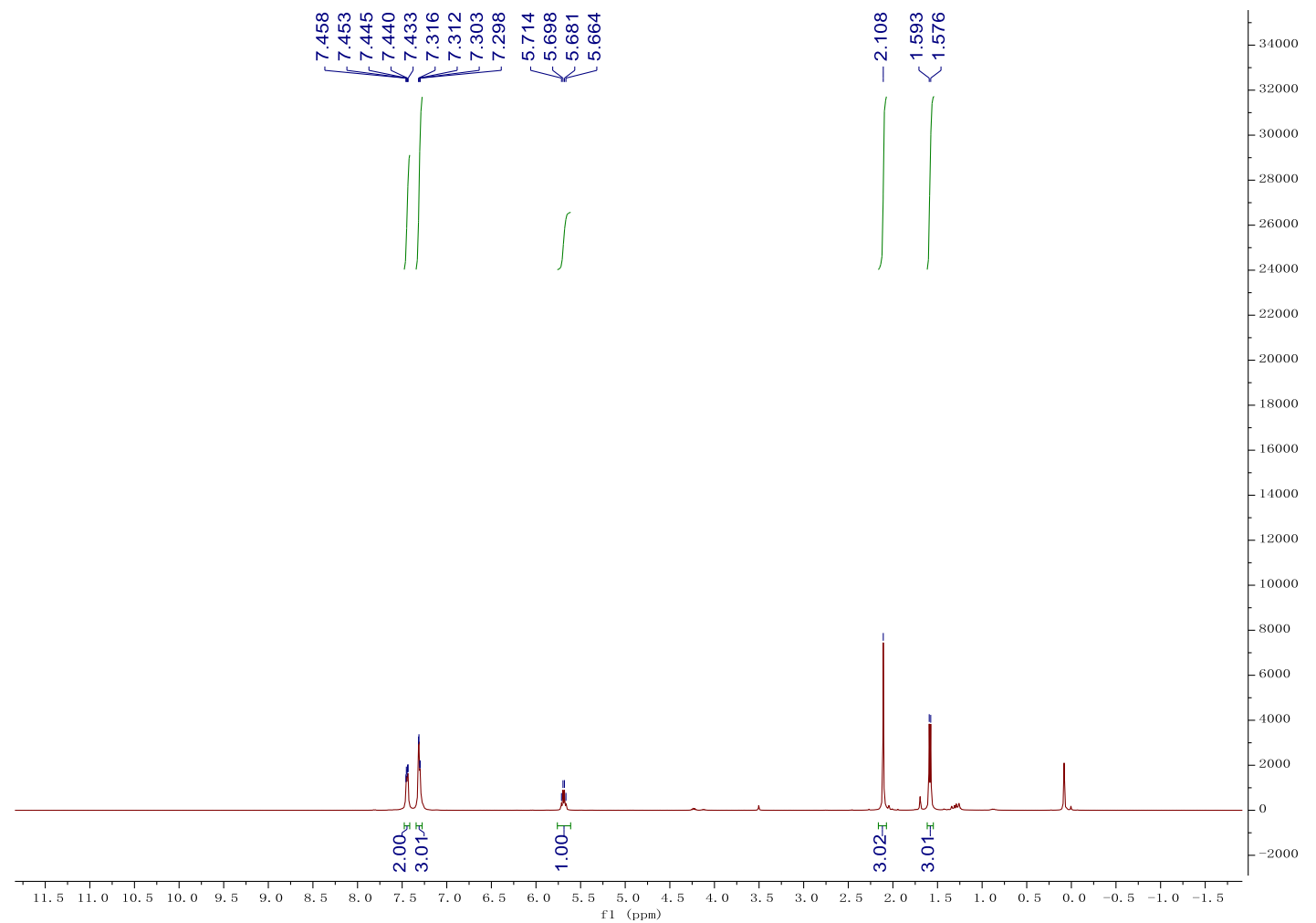



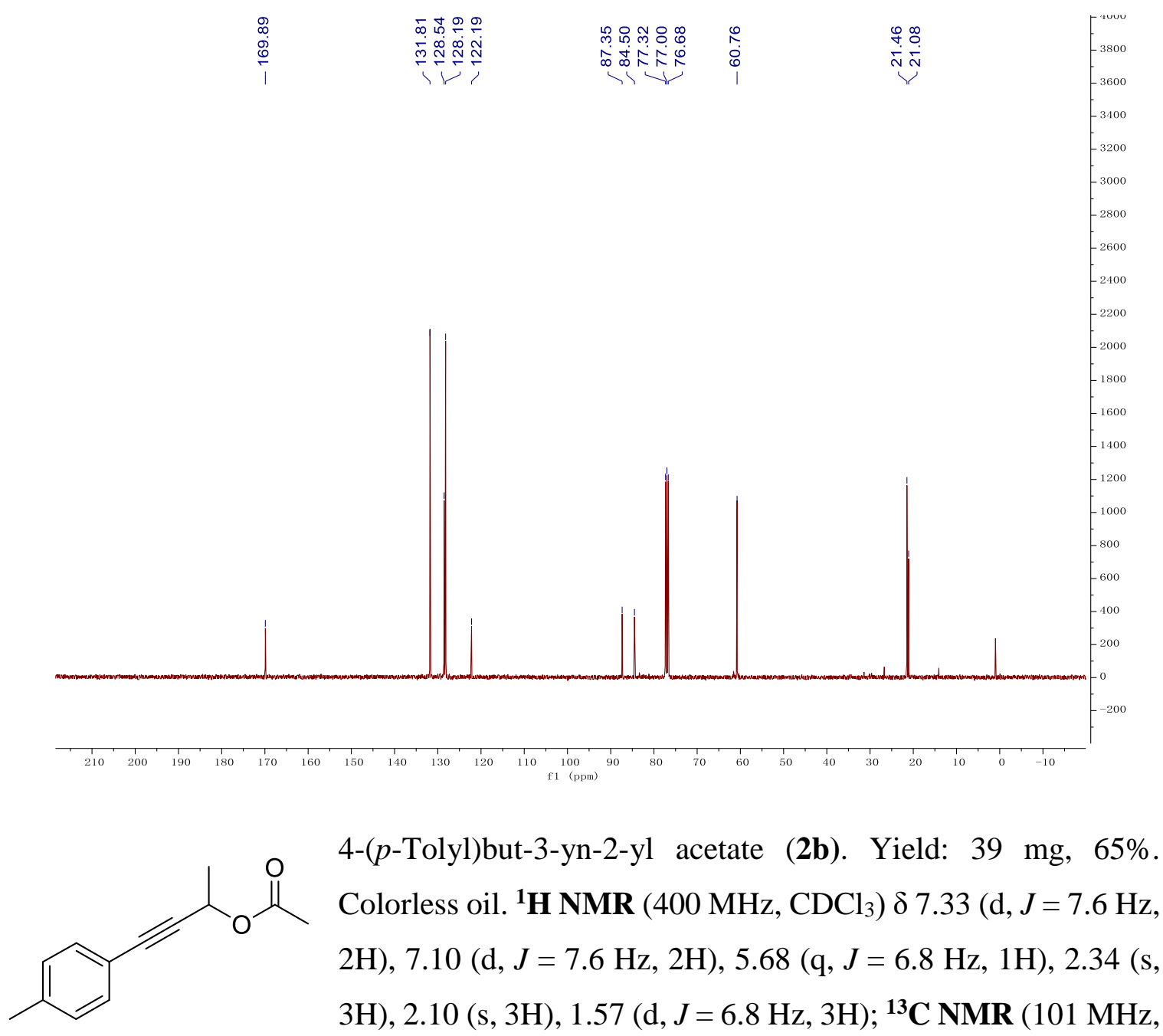
$\left.\mathrm{CDCl}_{3}\right) \delta 170.0,138.7,131.7,129.0,119.1,86.7,84.7,60.9,21.5,21.4,21.1 . \mathbf{I R}(\mathrm{KBr})$ : v 3031, 2990, 2871, 1738, 1448, 1233, 1035, 952, $818 \mathrm{~cm}^{-1}$. HRMS (ESI) calcd for $\left[\mathrm{C}_{13} \mathrm{H}_{14} \mathrm{O}_{2}+\mathrm{Na}\right]^{+}$requires 225.0886, found $225.0888\left[\mathrm{M}^{+}+\mathrm{Na}\right]$. 


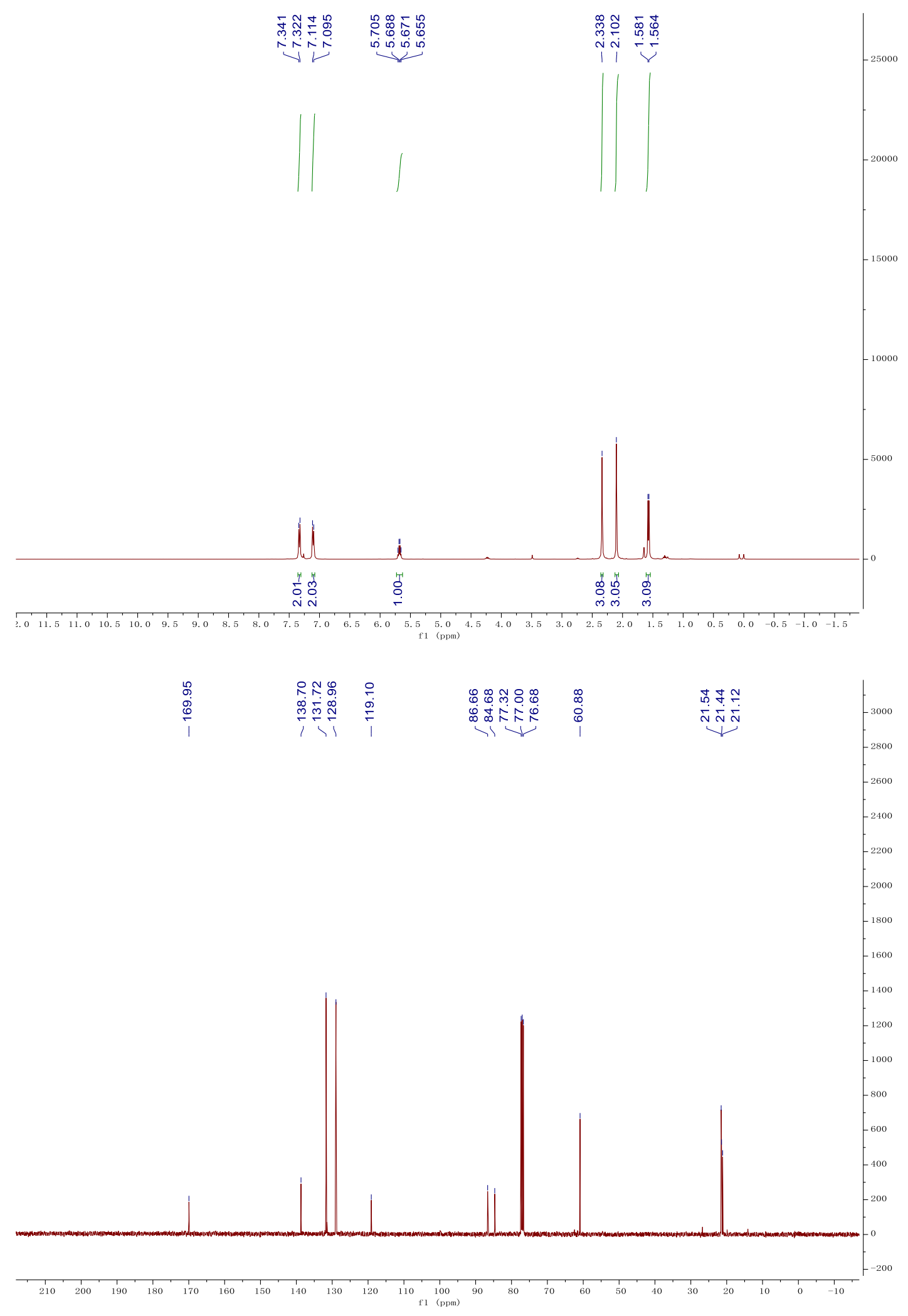




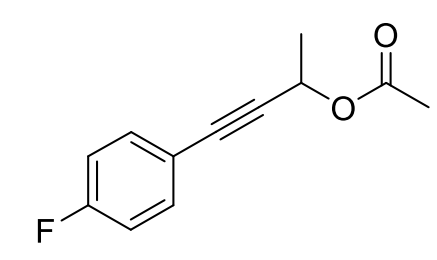

4-(4-Fluorophenyl)but-3-yn-2-yl acetat (2c). A known compound $^{[2]}$. Yield: $41 \mathrm{mg}, 67 \%$. Colorless oil. ${ }^{1} \mathbf{H}$ NMR $\left(400 \mathrm{MHz}, \mathrm{CDCl}_{3}\right) \delta$ 7.45-7.40 (m, 2H), $7.00(\mathrm{dd}, J=8.4$, $8.4 \mathrm{~Hz}, 2 \mathrm{H}), 5.56(\mathrm{q}, J=6.8 \mathrm{~Hz}, 1 \mathrm{H}), 2.11(\mathrm{~s}, 3 \mathrm{H}), 1.57$ (d, $J=6.8 \mathrm{~Hz}, 3 \mathrm{H}) ;{ }^{13} \mathbf{C ~ N M R}\left(101 \mathrm{MHz}, \mathrm{CDCl}_{3}\right) \delta 169.9,162.6\left(J_{\mathrm{CF}}=250.5 \mathrm{~Hz}\right), 133.7$ $\left(J_{\mathrm{CF}}=8.1 \mathrm{~Hz}\right), 118.3\left(J_{\mathrm{CF}}=4.0 \mathrm{~Hz}\right), 115.5\left(J_{\mathrm{CF}}=22.2 \mathrm{~Hz}\right), 87.1,83.5,60.7,21.4,21.1$; ${ }^{19}$ F NMR $\left(376 \mathrm{MHz}, \mathrm{CDCl}_{3}\right) \delta-110.39--110.46(\mathrm{~m}, 1 \mathrm{~F})$.

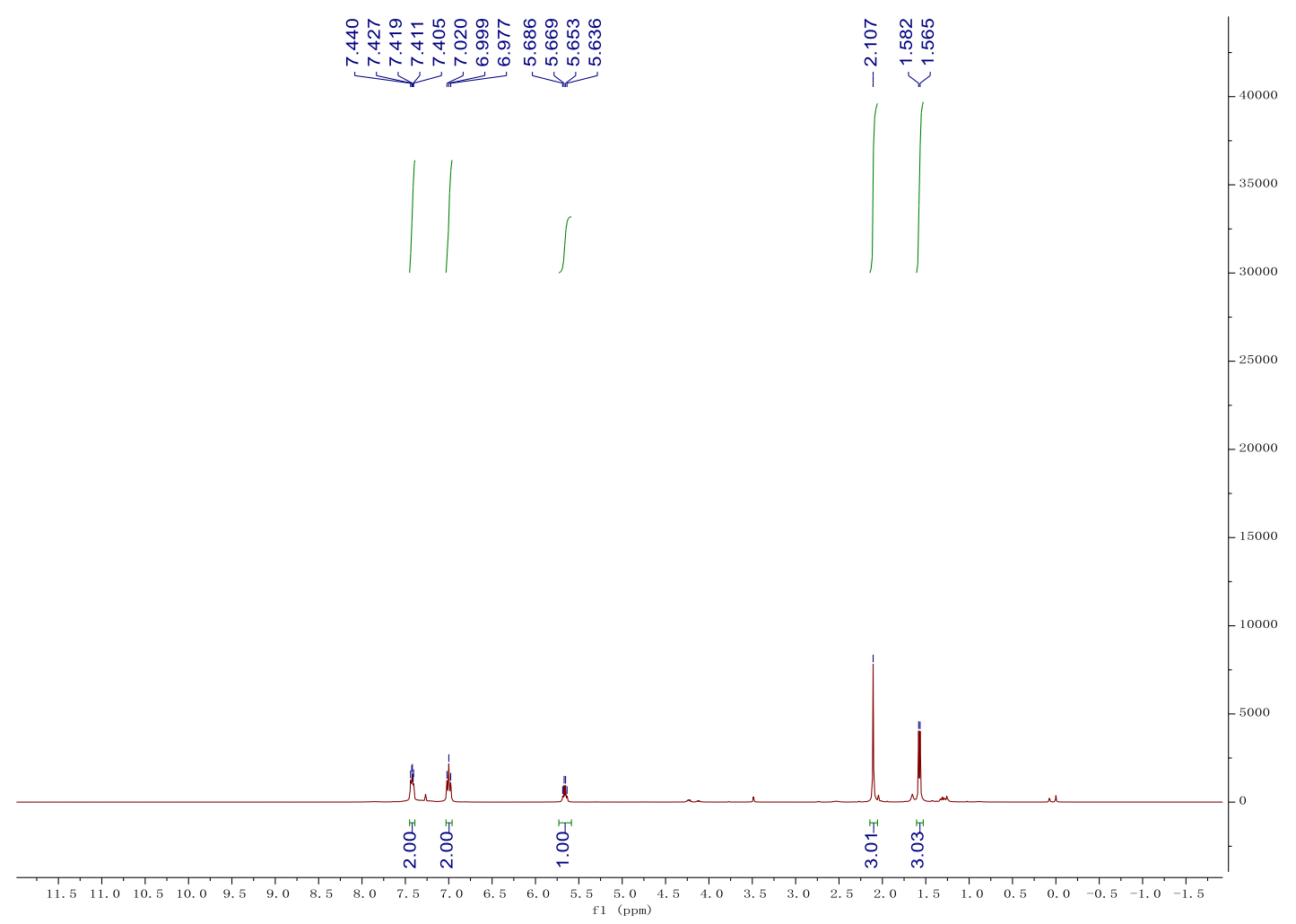



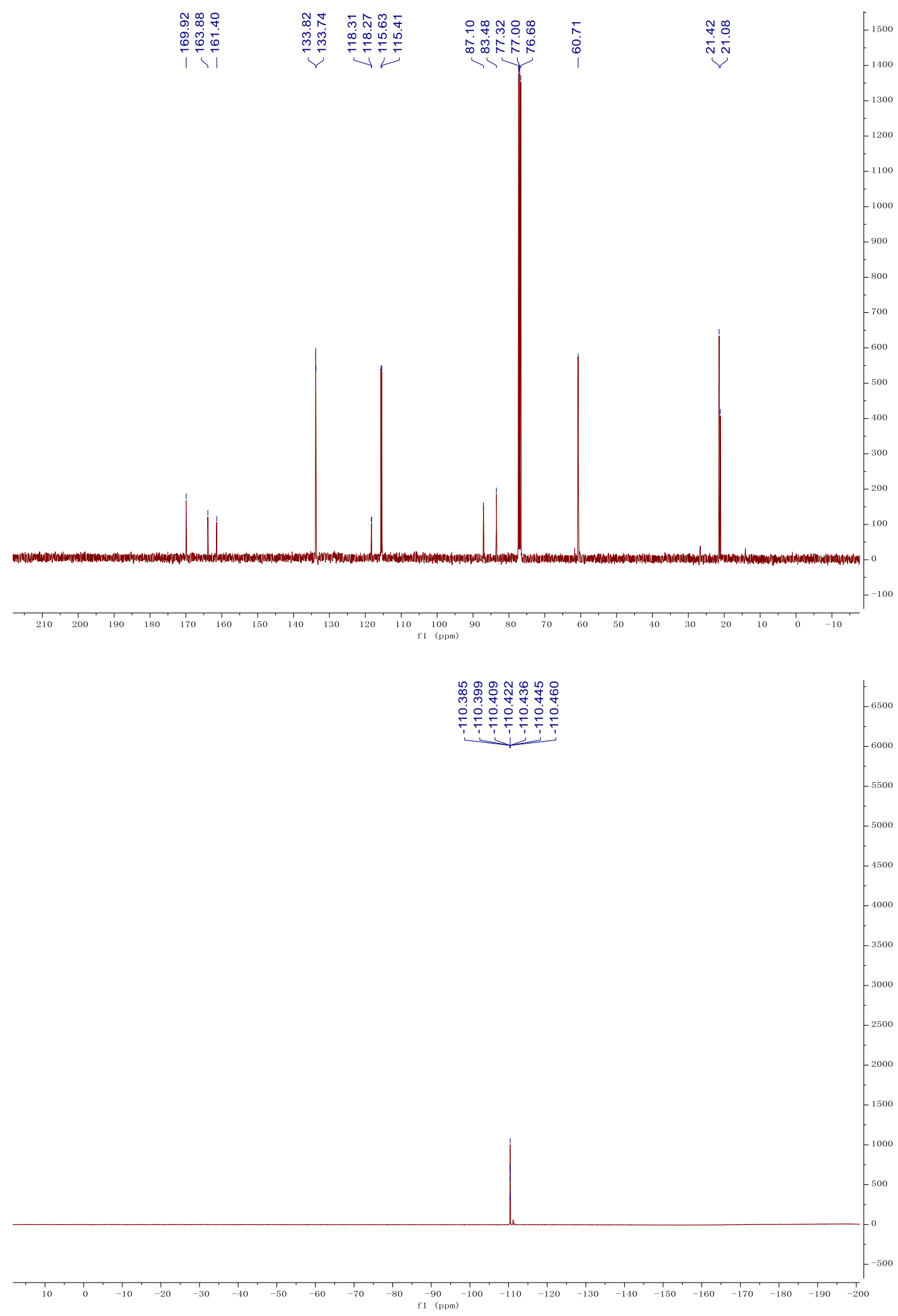


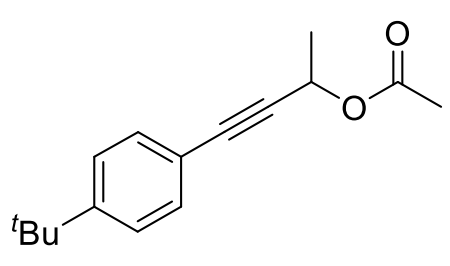

4-(4-(tert-Butyl)phenyl)but-3-yn-2-yl acetate (2d). Yield: $53 \mathrm{mg}, 70 \%$. Colorless oil. ${ }^{1} \mathbf{H}$ NMR $(400 \mathrm{MHz}$, CDCl3) $\delta 7.38(\mathrm{~d}, J=8.4 \mathrm{~Hz}, 2 \mathrm{H}), 7.32(\mathrm{~d}, J=8.0 \mathrm{~Hz}$, $2 \mathrm{H}), 5.68(\mathrm{q}, J=6.8 \mathrm{~Hz}, 1 \mathrm{H}), 2.10(\mathrm{~s}, 3 \mathrm{H}), 1.57(\mathrm{~d}, J=$

$6.4 \mathrm{~Hz}, 3 \mathrm{H}), 1.30$ (s, 9H); ${ }^{13} \mathrm{C}$ NMR (101 MHz, $\left.\mathrm{CDCl}_{3}\right) \delta 169.9,151.9,131.6,125.2$, 119.2, 86.7, 84.7, 60.9, 34.7, 31.1, 21.6, 21.1. IR (KBr): v 3038, 2963, 2871, 1744, 1461, 1233, 1035, 953, $838 \mathrm{~cm}^{-1}$. HRMS (ESI) calcd for $\left[\mathrm{C}_{16} \mathrm{H}_{20} \mathrm{O}_{2}+\mathrm{H}\right]^{+}$requires 245.1536, found $245.1538\left[\mathrm{M}^{+}+\mathrm{H}\right]$.

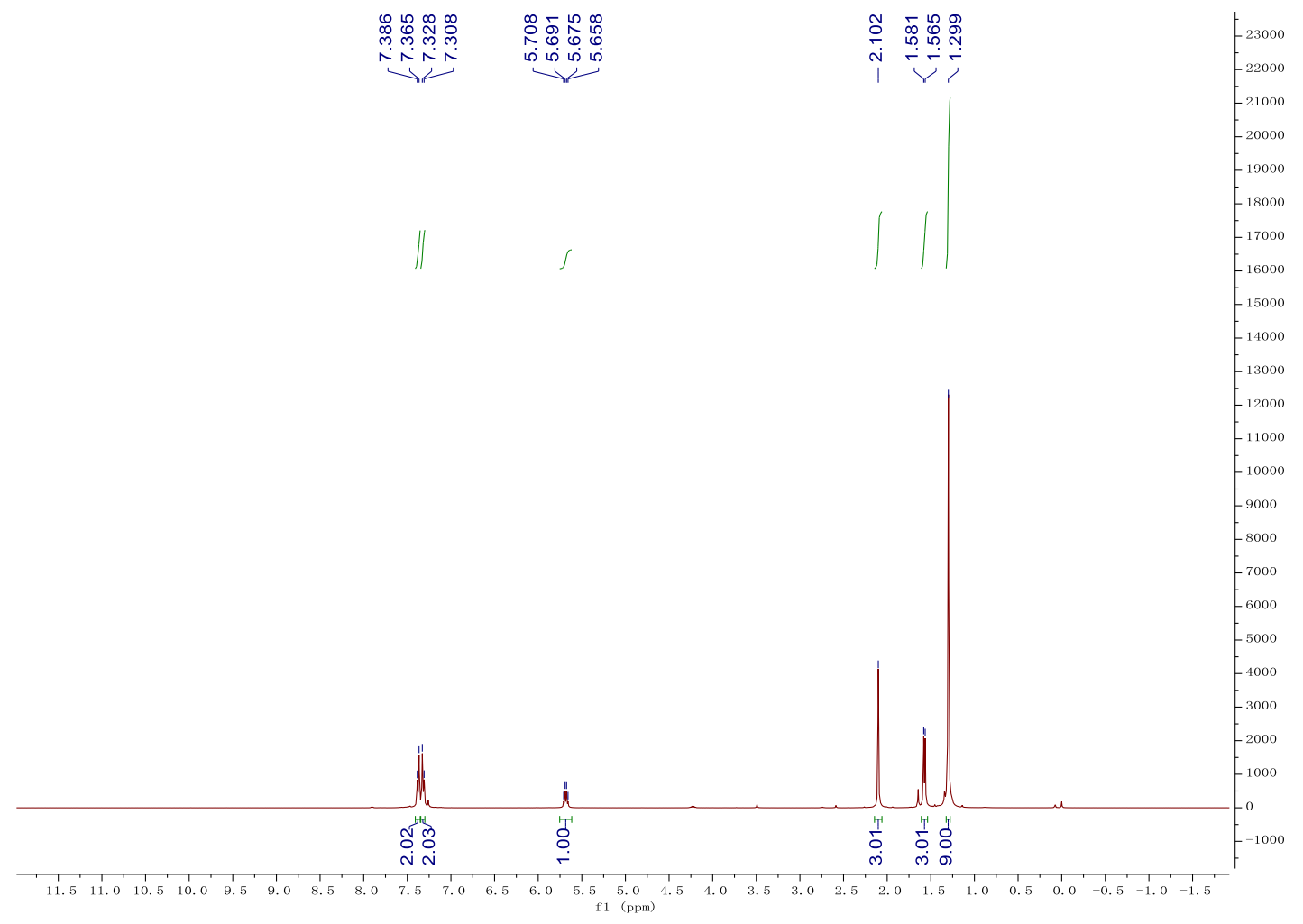




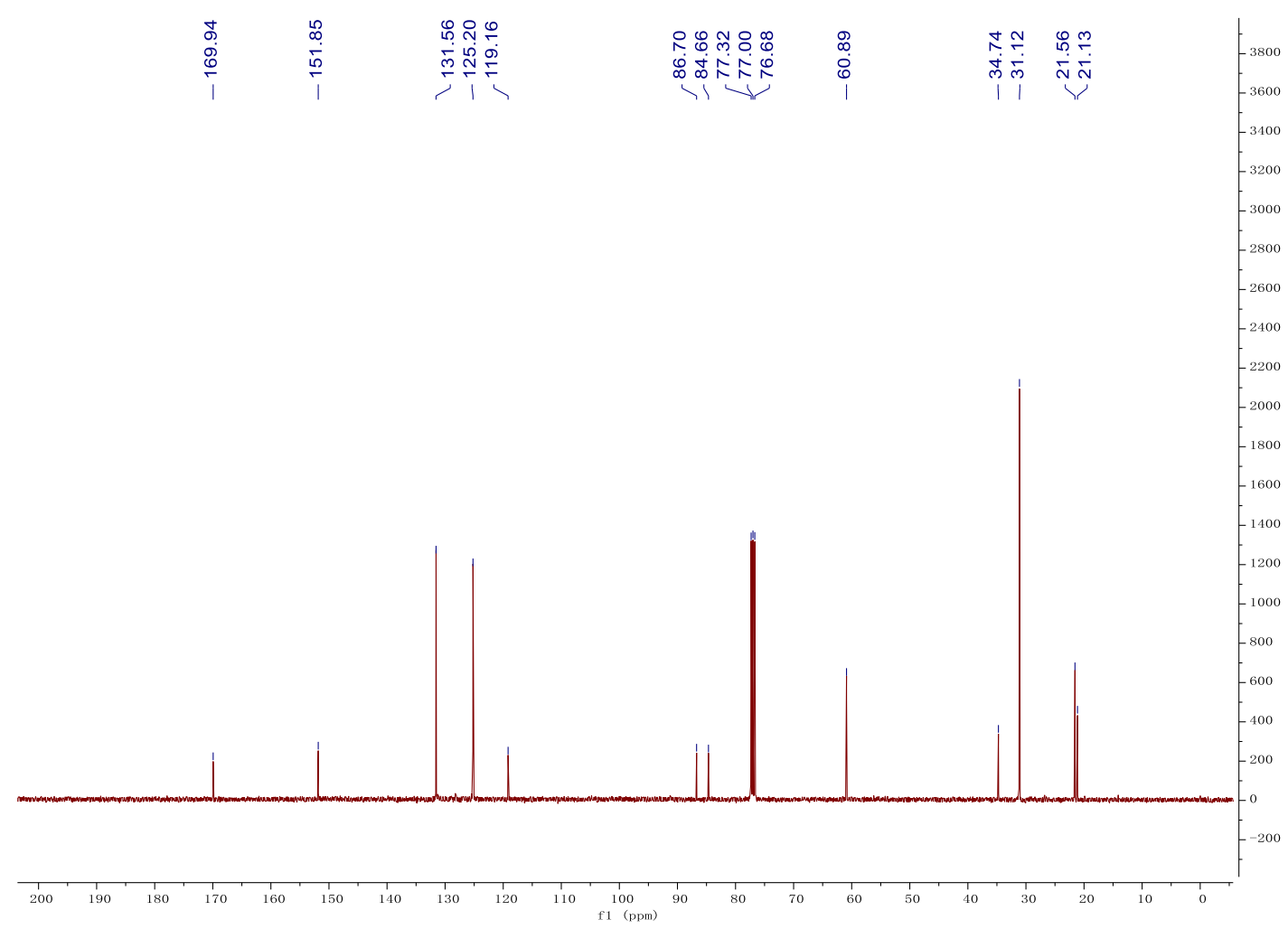

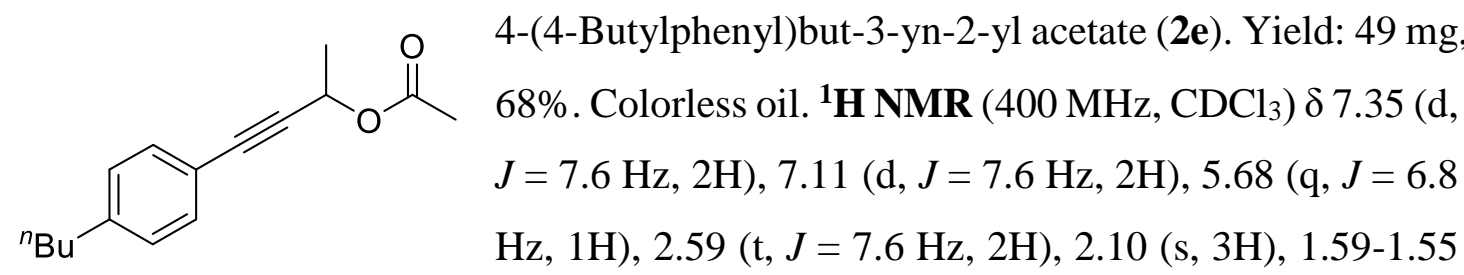
$(\mathrm{m}, 5 \mathrm{H}), 1.38-1.28(\mathrm{~m}, 2 \mathrm{H}), 0.91(\mathrm{t}, J=7.6 \mathrm{~Hz}, 3 \mathrm{H}) ;{ }^{13} \mathbf{C ~ N M R}\left(101 \mathrm{MHz}, \mathrm{CDCl}_{3}\right) \delta$ 169.9, 143.7, 131.7, 128.3, 128.3, 119.3, 86.7, 84.7, 60.9, 35.5, 33.3, 22.2 , 21.6, 21.1, 13.9. IR (KBr): v 3030, 2989, 2932, 2862, 1744, 1551, 1233, 1035, 953, $837 \mathrm{~cm}^{-1}$. HRMS (ESI) calcd for $\left[\mathrm{C}_{16} \mathrm{H}_{20} \mathrm{O}_{2}+\mathrm{Na}^{+}\right]$requires 267.1356, found $267.1358\left[\mathrm{M}^{+}+\mathrm{Na}\right]$. 


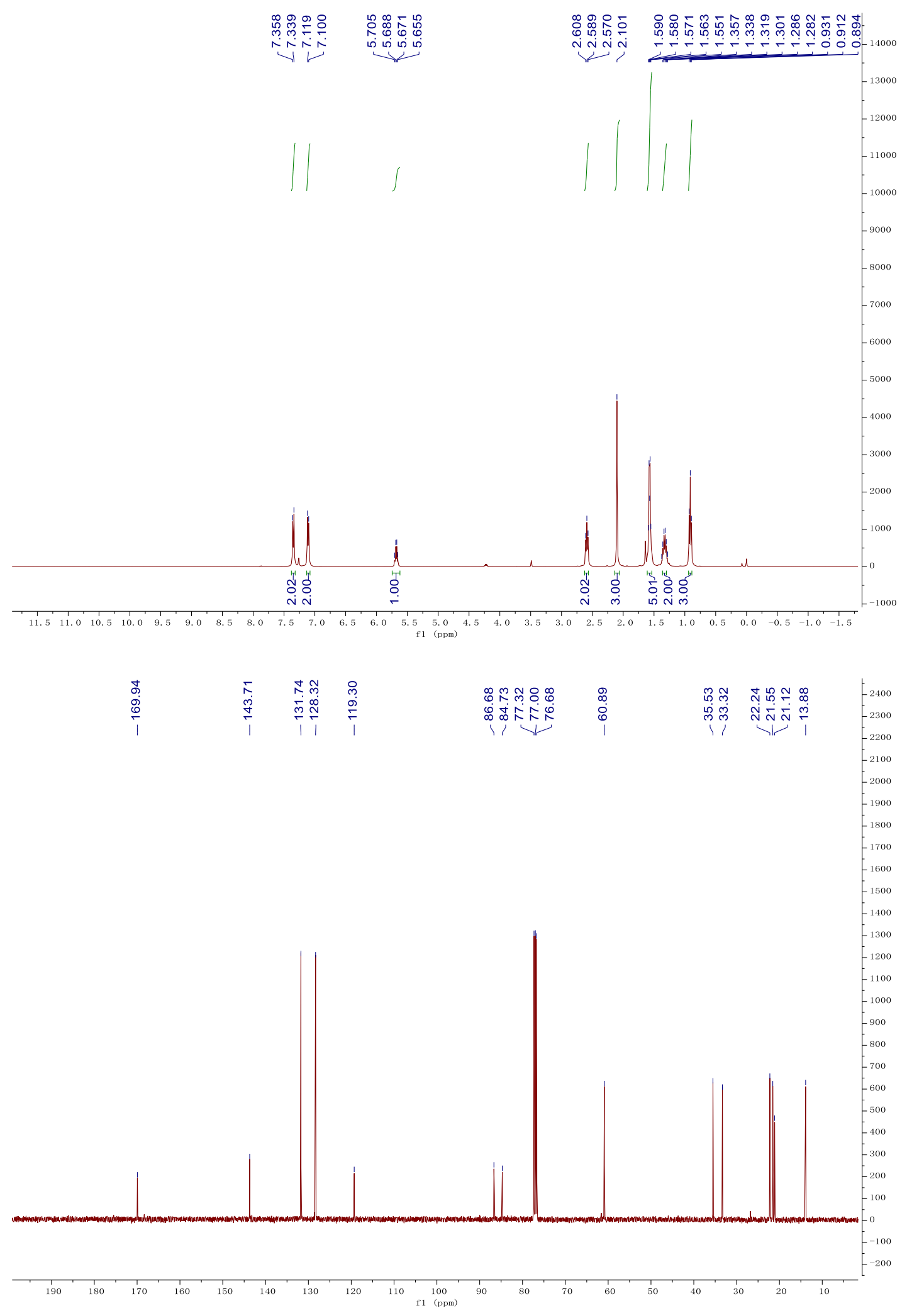




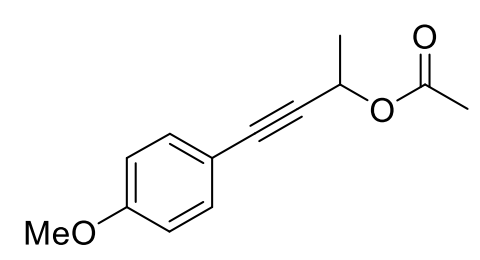

4-(4-Methoxyphenyl)but-3-yn-2-yl acetate (2f). A known $3 \mathrm{H}), 1.57(\mathrm{~d}, J=6.8 \mathrm{~Hz}, 3 \mathrm{H}) ;{ }^{13} \mathrm{C}$ NMR $\left(101 \mathrm{MHz}, \mathrm{CDCl}_{3}\right) \delta 170.0,159.8,133.3$, $114.3,113.8,86.0,84.5,61.0,55.2,21.6,21.1$.

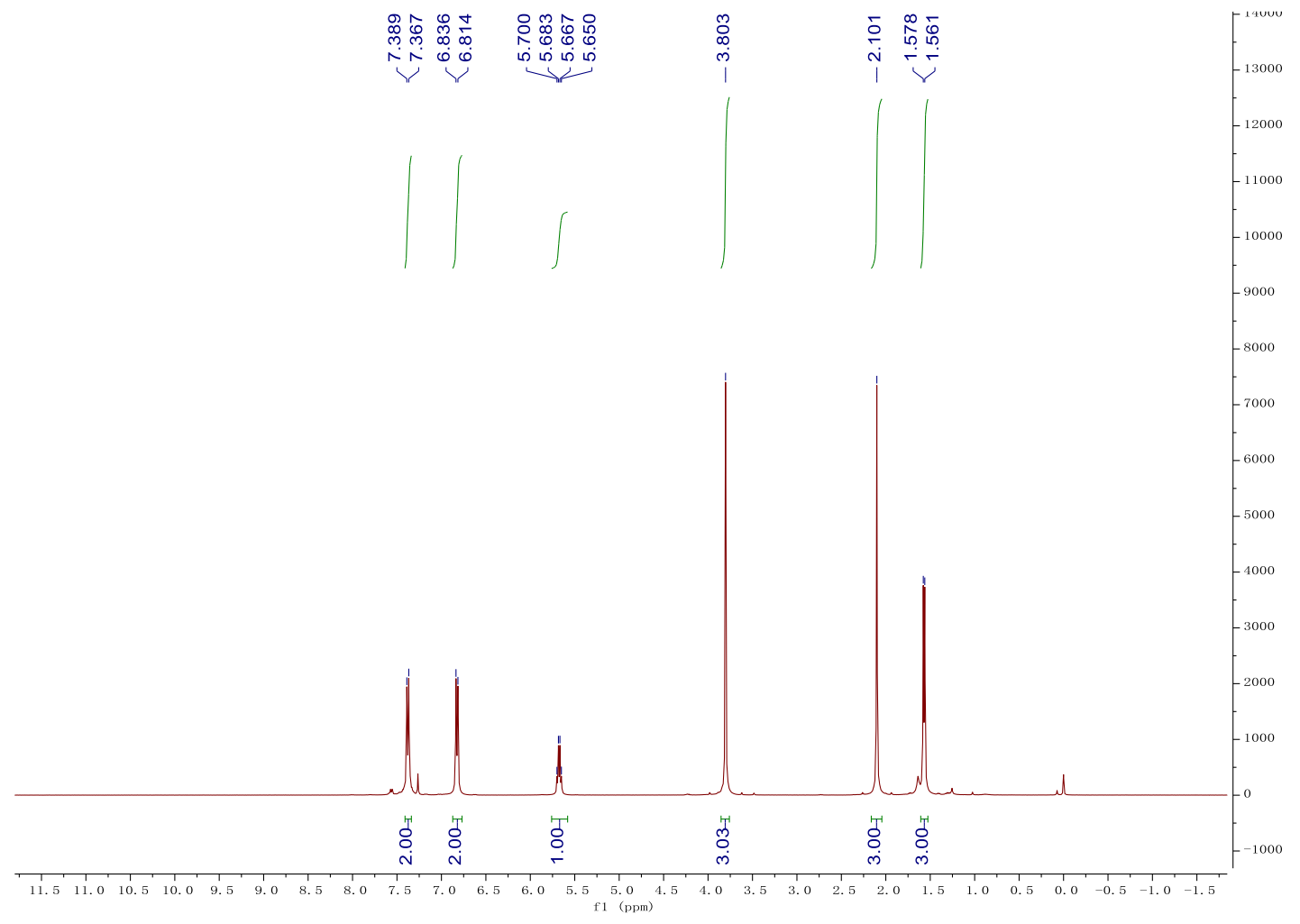




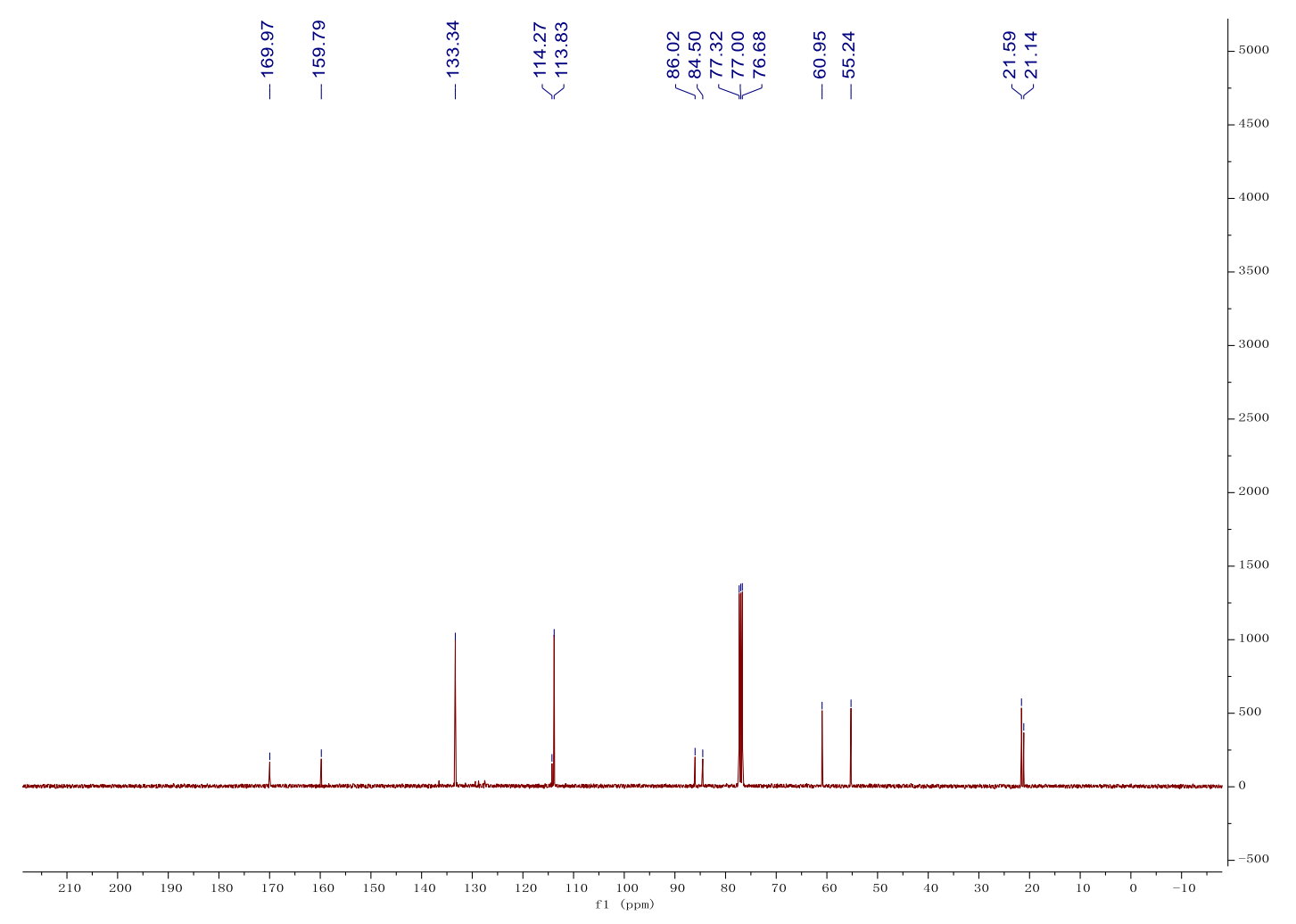

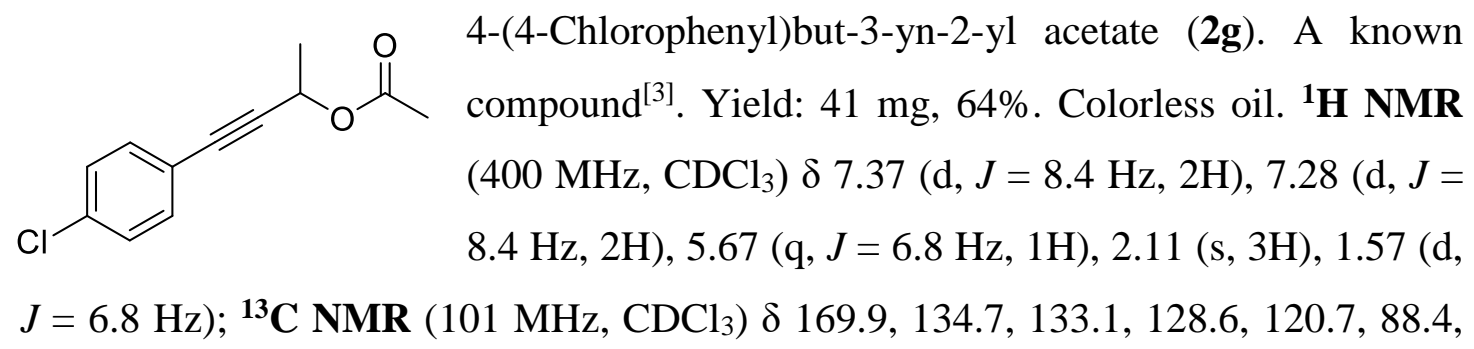
83.4, 60.7, 21.4, 21.1. 

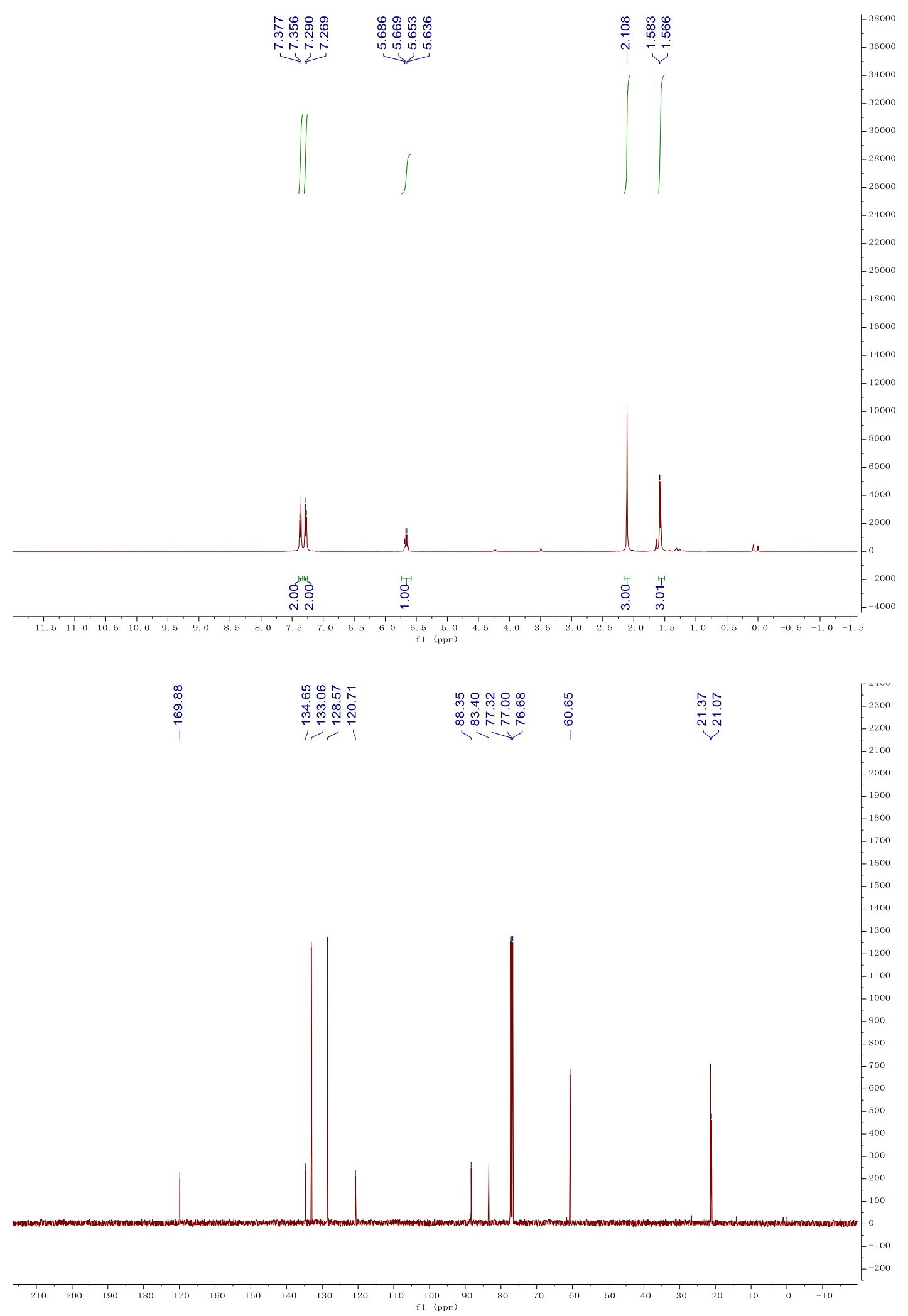


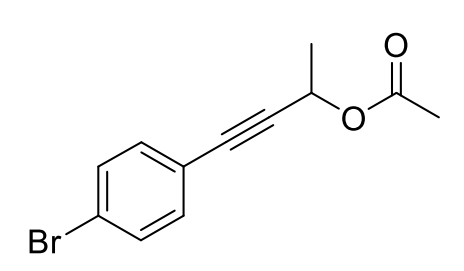

4-(4-Bromophenyl)but-3-yn-2-yl acetate (2h) Yield: $47 \mathrm{mg}$, 61\%. White solid. M.p. $41-42{ }^{\circ} \mathrm{C} .{ }^{\mathbf{1}} \mathbf{H}$ NMR $\left(400 \mathrm{MHz}, \mathrm{CDCl}_{3}\right)$ $\delta 7.44(\mathrm{~d}, J=8.0 \mathrm{~Hz}, 2 \mathrm{H}), 7.30(\mathrm{~d}, J=8.4 \mathrm{~Hz}, 2 \mathrm{H}), 5.65(\mathrm{q}, J$ $=6.8 \mathrm{~Hz}, 1 \mathrm{H}), 2.11(\mathrm{~s}, 3 \mathrm{H}), 1.57(\mathrm{~d}, J=6.8 \mathrm{~Hz}, 3 \mathrm{H}) ;{ }^{13} \mathbf{C} \mathbf{N M R}$ $\left(101 \mathrm{MHz} \mathrm{CDCl}_{3}\right) \delta 170.0,133.3,131.5,122.9,121.2,88.5,83.5,60.7,21.4,21.1 . \mathbf{I R}$ $(\mathrm{KBr}): v 2990,2937,1745,1486,1371,1232,1035,953,825,544 \mathrm{~cm}^{-1}$. HRMS (ESI) calcd for $\left[\mathrm{C}_{16} \mathrm{H}_{20} \mathrm{O}_{2}+\mathrm{Na}\right]^{+}$requires 288.9835 , found $288.9837\left[\mathrm{M}^{+}+\mathrm{Na}\right]$.

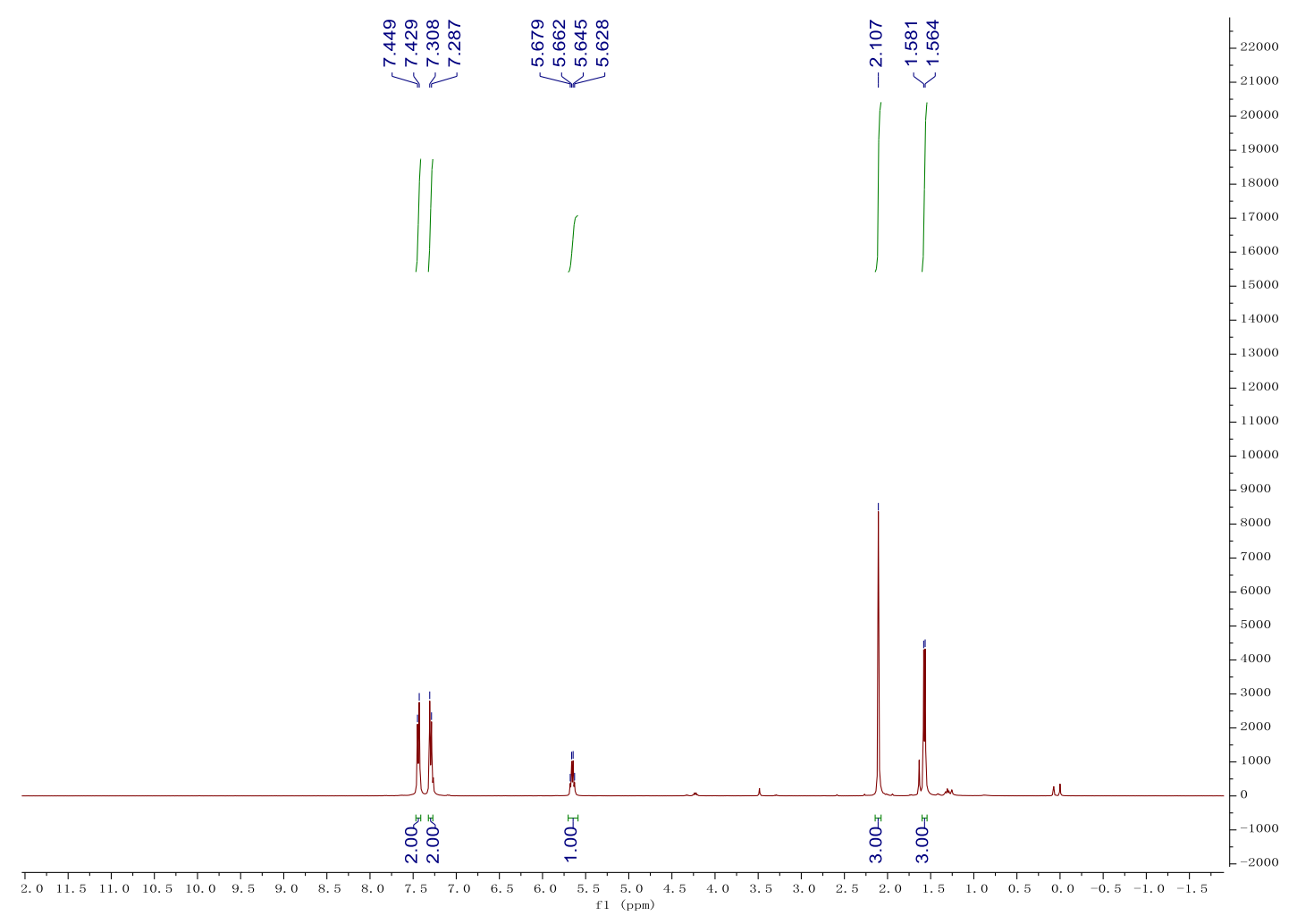



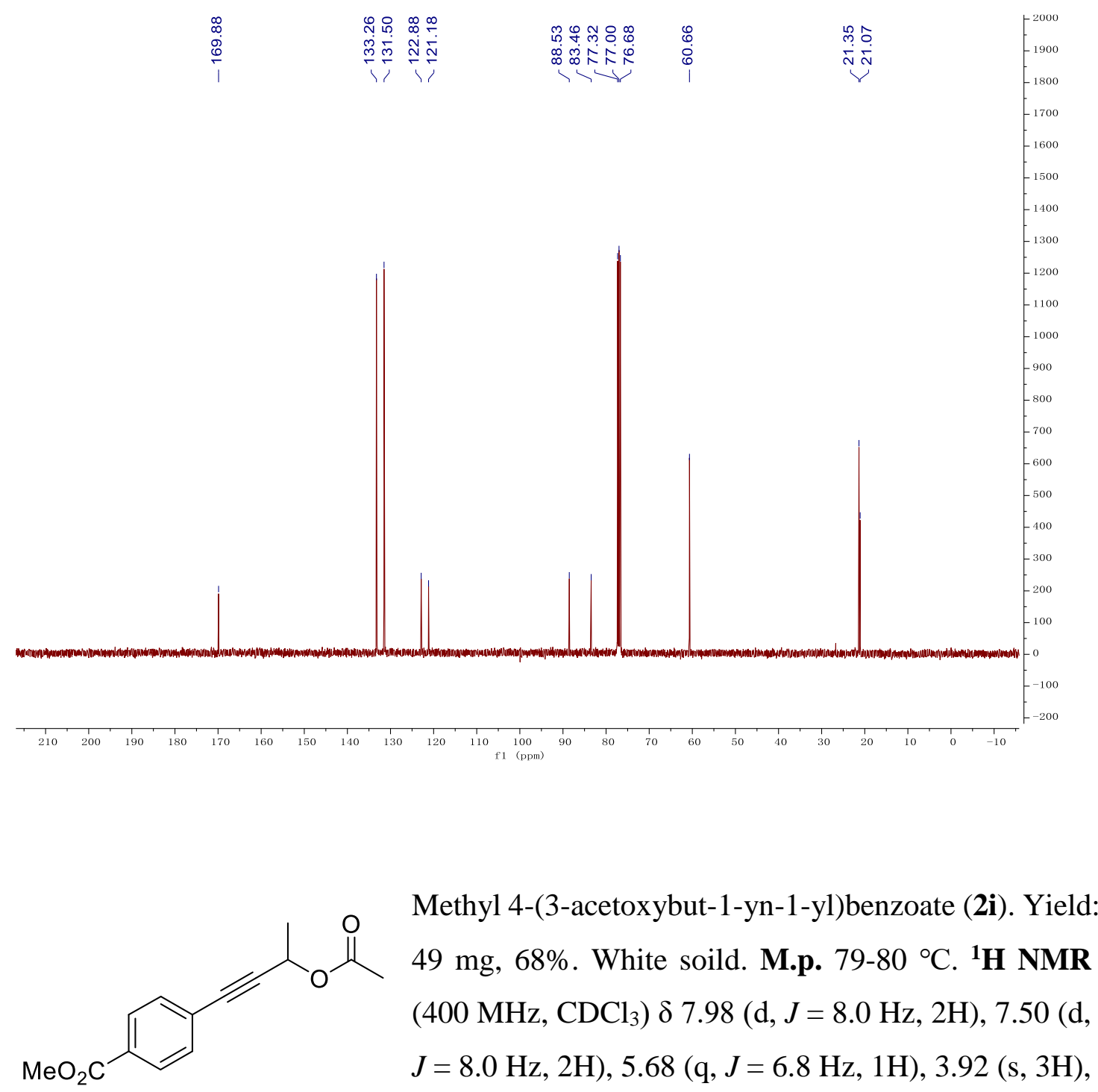
$2.12(\mathrm{~s}, 3 \mathrm{H}), 1.59$ (d, $J=6.8 \mathrm{~Hz}, 3 \mathrm{H}) ;{ }^{13} \mathrm{C}$ NMR $\left(101 \mathrm{MHz}, \mathrm{CDCl}_{3}\right) \delta$ 169.9, 166.4, 131.7, 129.9, 129.4, 126.9, 90.3, 83.7, 60.6, 52.2, 21.3, 21.1. IR (KBr): v 2986, 2936, $2855,1739,1445,1280,1037,957,768 \mathrm{~cm}^{-1}$. HRMS (ESI) calcd for $\left[\mathrm{C}_{14} \mathrm{H}_{14} \mathrm{O}_{4}+\mathrm{Na}\right]^{+}$ requires 269.0784 , found $269.0786\left[\mathrm{M}^{+}+\mathrm{Na}\right]$. 

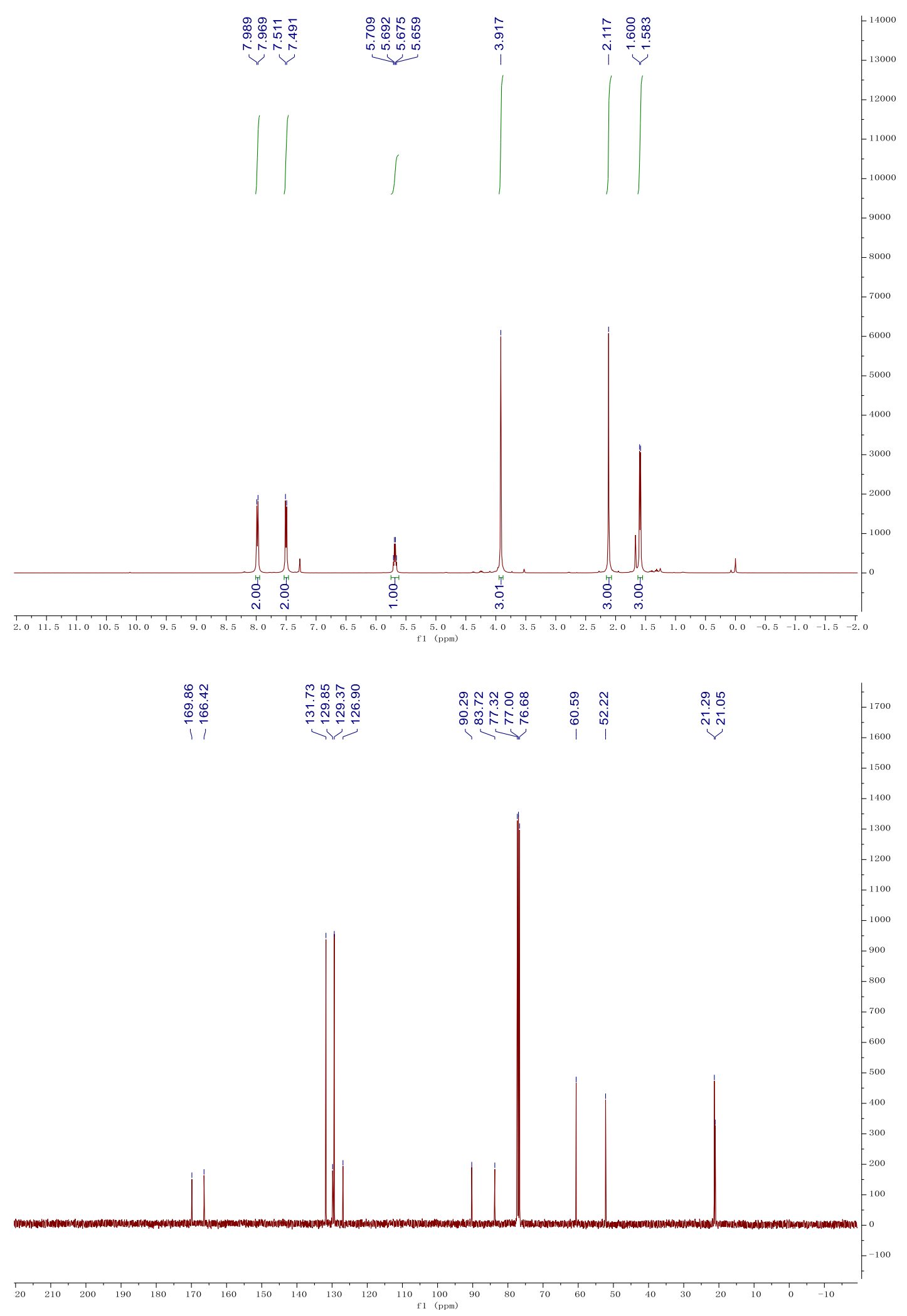


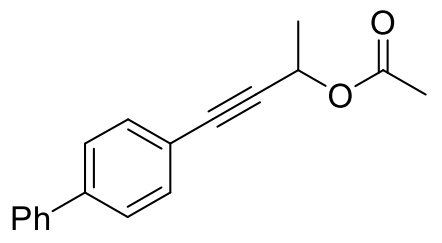

4-([1,1'-Biphenyl]-4-yl)but-3-yn-2-yl acetate (2j). Yield: $51 \mathrm{mg}, 64 \%$. White solid. M.p. $77-78^{\circ} \mathrm{C} .{ }^{1} \mathbf{H}$ NMR (400 $\left.\mathrm{MHz}, \mathrm{CDCl}_{3}\right) \delta$ 7.60-7.49 (m, 6H), $7.44(\mathrm{dd}, J=7.6,7.6$ $\mathrm{Hz}, 2 \mathrm{H}), 7.38-7.33(\mathrm{~m}, 1 \mathrm{H}), 5.71(\mathrm{q}, J=6.4 \mathrm{~Hz}, 1 \mathrm{H}), 2.12$ $(\mathrm{s}, 3 \mathrm{H}), 1.60(\mathrm{~d}, J=6.4 \mathrm{~Hz}, 3 \mathrm{H}) ;{ }^{13} \mathbf{C} \mathbf{N M R}\left(101 \mathrm{MHz}, \mathrm{CDCl}_{3}\right) \delta$ 167.0, 141.3, 140.2, 132.3, 128.8, 127.7, 127.0, 126.9, 121.1, 88.0, 84.4, 60.9, 21.5, $21.1 \mathrm{~cm}^{-1}$. IR (KBr): v 3031, 2927, 2853, 1738, 1448, 1259, 1036, 842, $764 \mathrm{~cm}^{-1}$. HRMS (ESI) calcd for $\left[\mathrm{C}_{18} \mathrm{H}_{16} \mathrm{O}_{2}+\mathrm{H}\right]^{+}$requires 265.1223, found $265.1225\left[\mathrm{M}^{+}+\mathrm{H}\right]$.

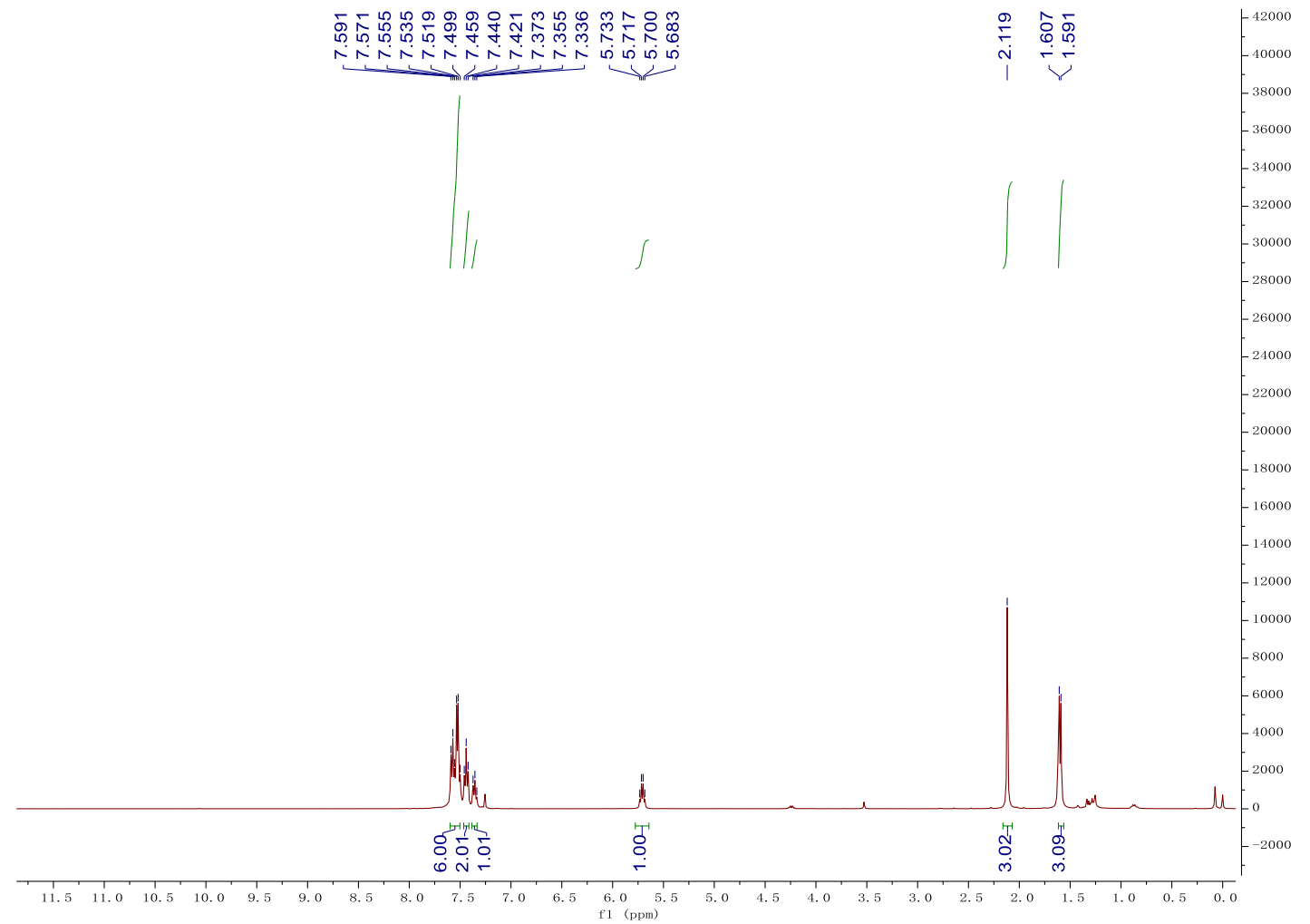



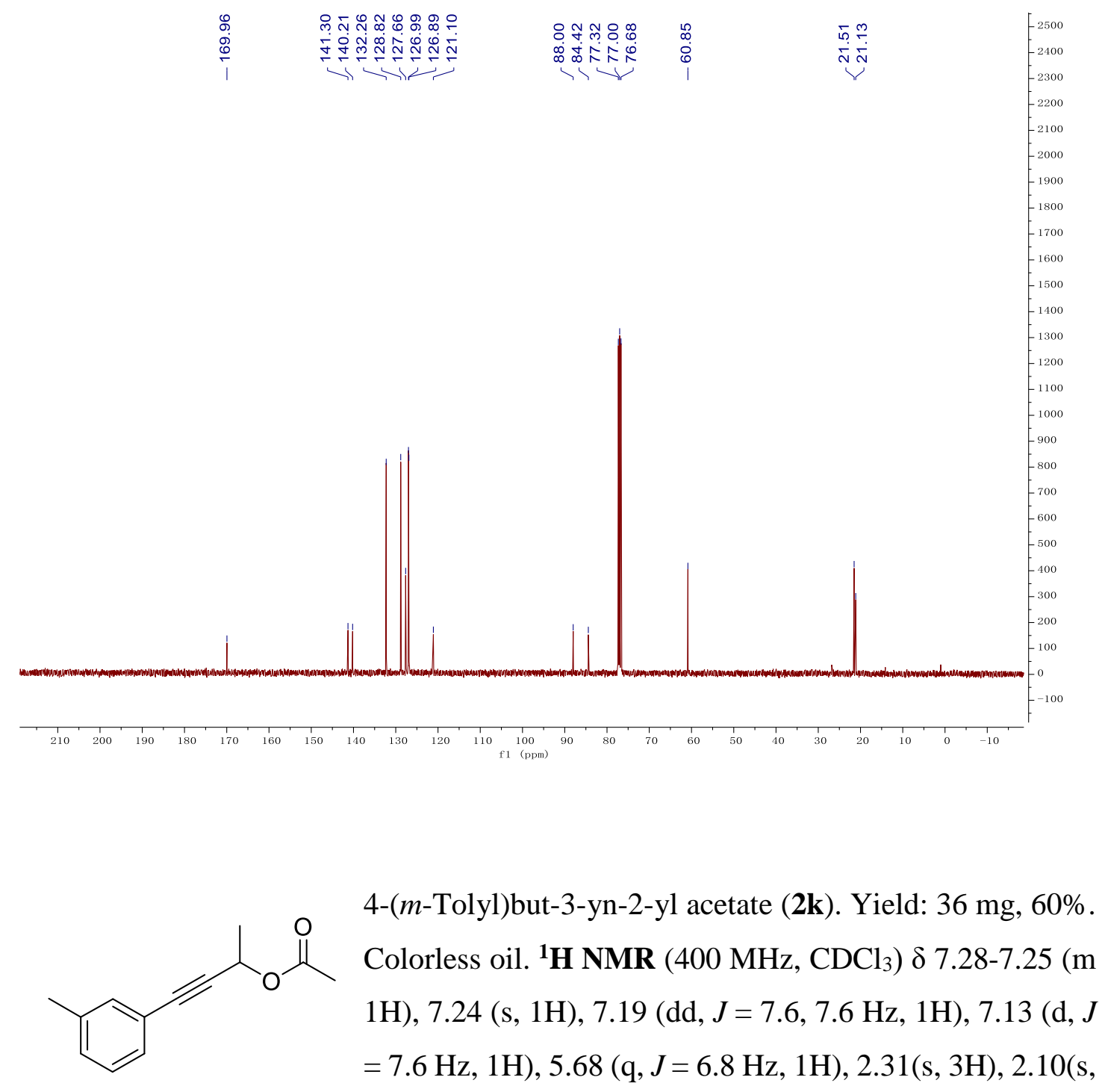

3H), $1.57(\mathrm{~d}, J=6.4 \mathrm{~Hz}, 3 \mathrm{H}) ;{ }^{13} \mathbf{C}$ NMR $\left(101 \mathrm{MHz}, \mathrm{CDCl}_{3}\right) \delta 169.9,137.9,132.4$, 129.4, 128.9, 128.1, 122.0, 87.0, 84.7, 60.8, 21.5, 21.1, 21.1. IR (KBr): v 3031, 2990, 2870, 1744, 1449, 1234, 1038, 959, $787 \mathrm{~cm}^{-1}$. HRMS (ESI) calcd for $\left[\mathrm{C}_{13} \mathrm{H}_{14} \mathrm{O}_{2}+\mathrm{Na}\right]^{+}$ requires 225.0886 , found $225.0888\left[\mathrm{M}^{+}+\mathrm{Na}\right]$. 

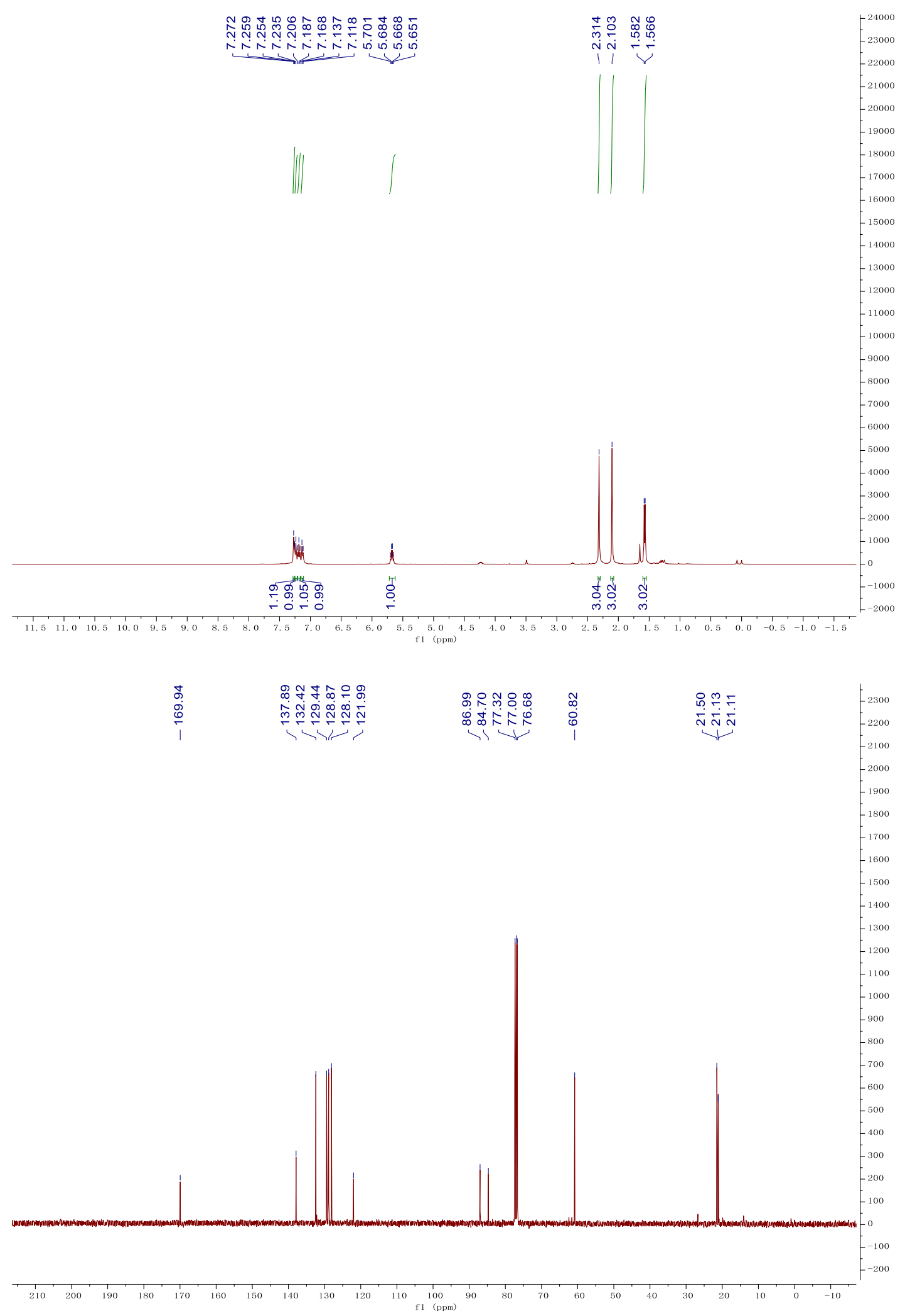


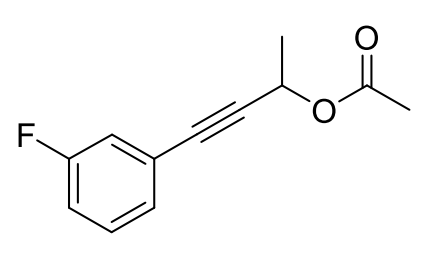

4-(3-Fluorophenyl)but-3-yn-2-yl acetate (2l). Yield: $38 \mathrm{mg}$, $62 \%$. Colorless oil. ${ }^{1} \mathbf{H}$ NMR $\left(400 \mathrm{MHz}, \mathrm{CDCl}_{3}\right.$,) $\delta$ 7.28$7.20(\mathrm{~m}, 2 \mathrm{H}), 7.14(\mathrm{~d}, J=9.6 \mathrm{~Hz}, 1 \mathrm{H}), 7.03(\mathrm{dd}, J=8.4$, $8.4 \mathrm{~Hz}, 1 \mathrm{H}), 5.67(\mathrm{q}, J=6.8 \mathrm{~Hz}, 1 \mathrm{H}), 2.11(\mathrm{~s}, 3 \mathrm{H}), 1.58(\mathrm{~d}$, $J=6.8 \mathrm{~Hz}, 3 \mathrm{H}) ;{ }^{13} \mathbf{C} \mathbf{N M R}\left(101 \mathrm{MHz}, \mathrm{CDCl}_{3}\right) \delta 169.9,162.2\left(J_{\mathrm{CF}}=247.5 \mathrm{~Hz}\right), 129.8$ $\left(J_{\mathrm{CF}}=9.1 \mathrm{~Hz}\right), 127.7\left(J_{\mathrm{CF}}=3.0 \mathrm{~Hz}\right), 124.1\left(J_{\mathrm{CF}}=9.1 \mathrm{~Hz}\right), 118.6\left(J_{\mathrm{CF}}=23.2 \mathrm{~Hz}\right), 116.0$ $\left(J_{\mathrm{CF}}=22.2 \mathrm{~Hz}\right), 88.3,83.3\left(J_{\mathrm{CF}}=3.0 \mathrm{~Hz}\right), 60.6,21.3,21.1 .{ }^{19} \mathbf{F ~ N M R}\left(376 \mathrm{MHz}, \mathrm{CDCl}_{3}\right)$ $\delta$-112.90 - -113.00 (m). IR (KBr): v 3075, 2991, 2939, 1744, 1433, 1234, 1037, 968, $787 \mathrm{~cm}^{-1}$. HRMS (ESI) calcd for $\left[\mathrm{C}_{12} \mathrm{H}_{11} \mathrm{FO}_{2}+\mathrm{H}\right]^{+}$requires 207.0816, found 207.0818 $\left[\mathrm{M}^{+}+\mathrm{H}\right]$.

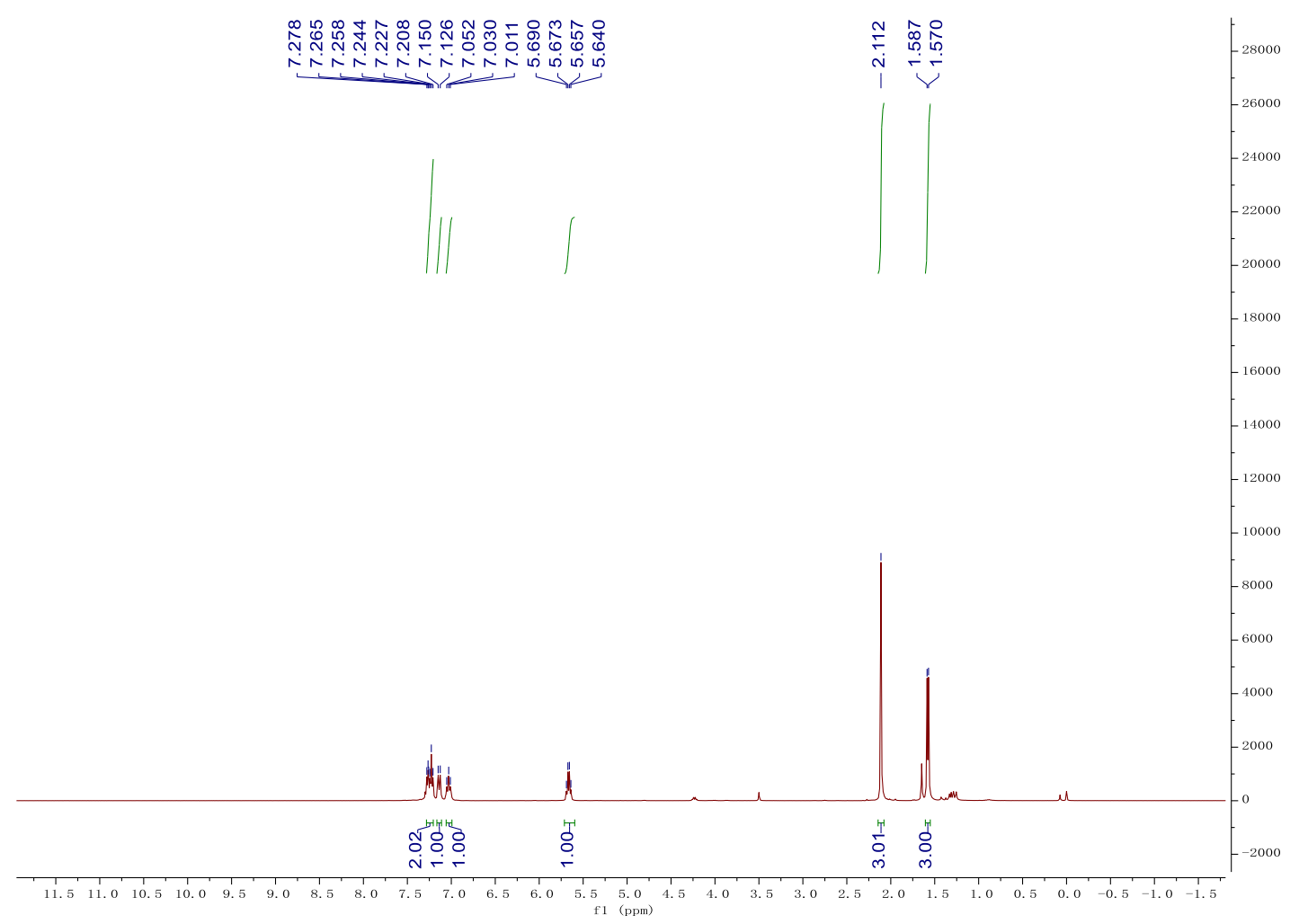




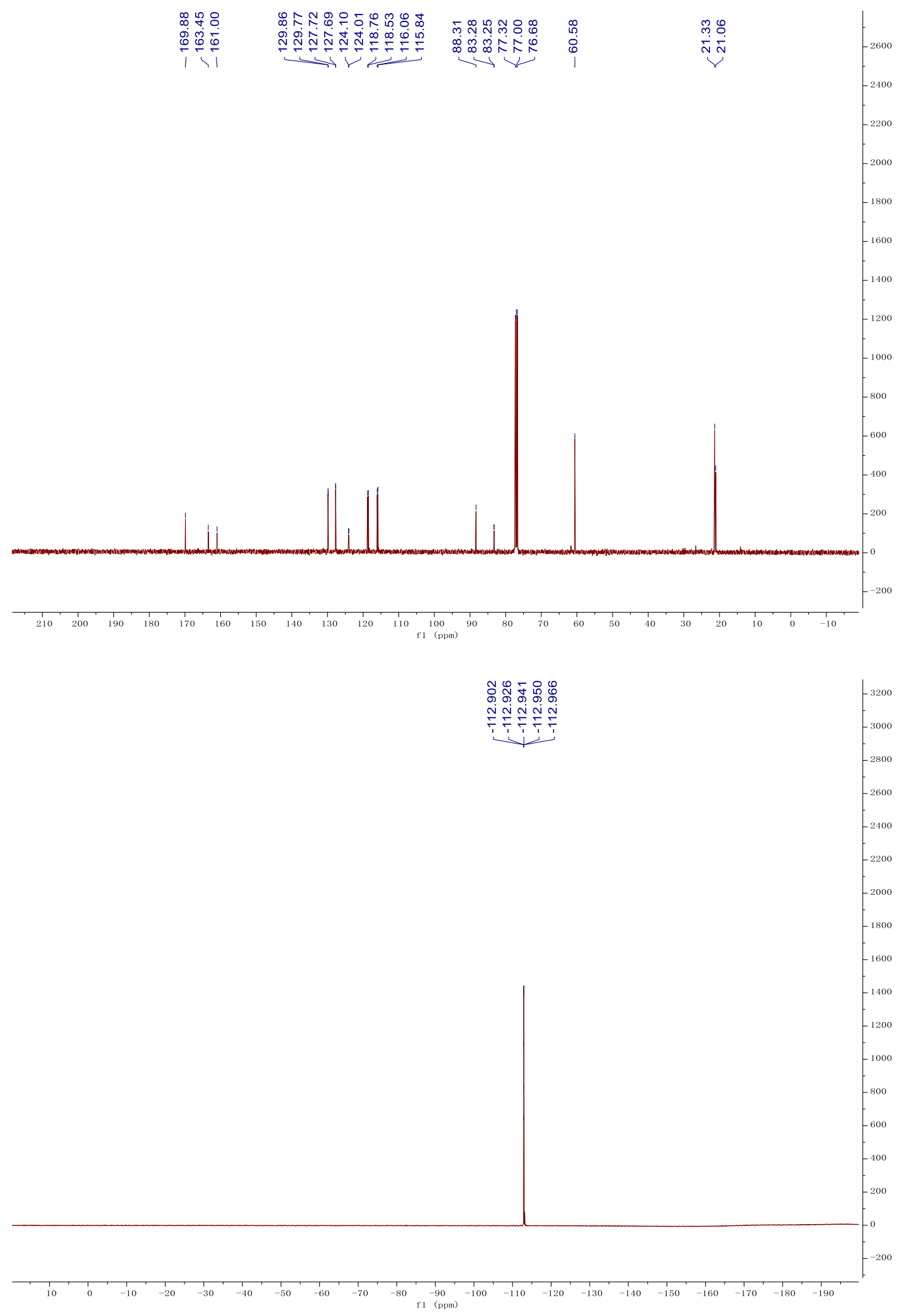




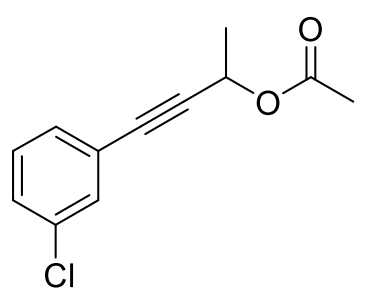

4-(3-Chlorophenyl)but-3-yn-2-yl acetate (2m). Yield: $41 \mathrm{mg}, 62 \%$. Colorless oil. ${ }^{1} \mathbf{H}$ NMR $\left(400 \mathrm{MHz}, \mathrm{CDCl}_{3}\right) \delta 7.43(\mathrm{~s}, 1 \mathrm{H}), 7.31(\mathrm{dd}$, $J=7.6,7.6 \mathrm{~Hz}, 2 \mathrm{H}), 7.23(\mathrm{dd}, J=7.6,7.6 \mathrm{~Hz}, 1 \mathrm{H}), 5.66(\mathrm{q}, J=$ $6.4 \mathrm{~Hz}, 1 \mathrm{H}), 2.11(\mathrm{~s}, 3 \mathrm{H}), 1.57(\mathrm{~d}, J=6.8 \mathrm{~Hz}, 3 \mathrm{H}) ;{ }^{13} \mathrm{C}$ NMR $(101$ $\left.\mathrm{MHz}, \mathrm{CDCl}_{3}\right) \delta 169.9,134.1,131.7,129.9,129.5,128.9,123.9$, 88.6, 83.1, 60.6, 21.33, 21.1. IR (KBr): v 3069, 2991, 2937, 1745, 1475, 1232, 1085, 1037, $786 \mathrm{~cm}^{-1}$. HRMS (ESI) calcd for $\left[\mathrm{C}_{12} \mathrm{H}_{11} \mathrm{ClO}_{2}+\mathrm{Na}\right]^{+}$requires 245.0340, found $245.0342\left[\mathrm{M}^{+}+\mathrm{Na}\right]$.

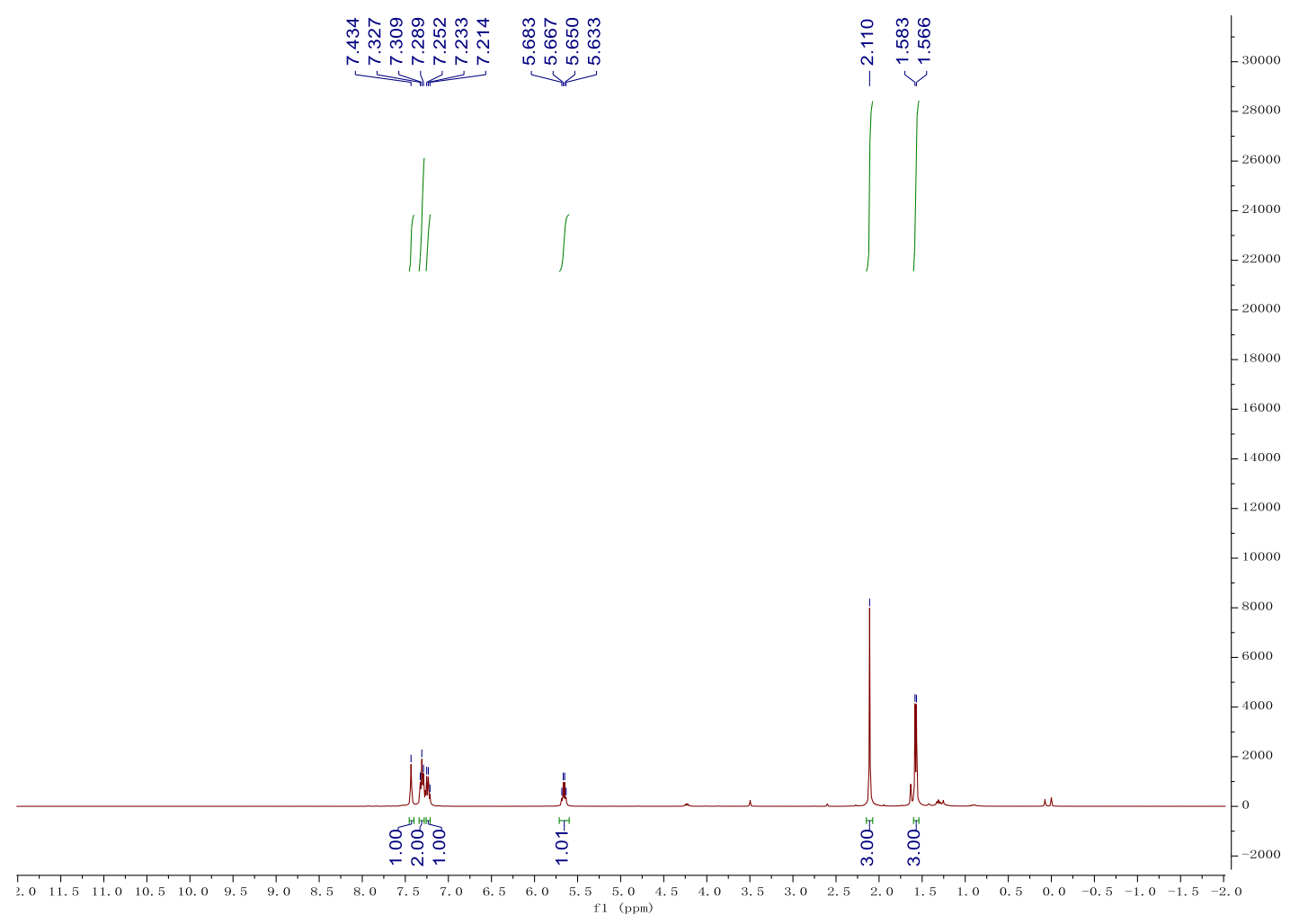



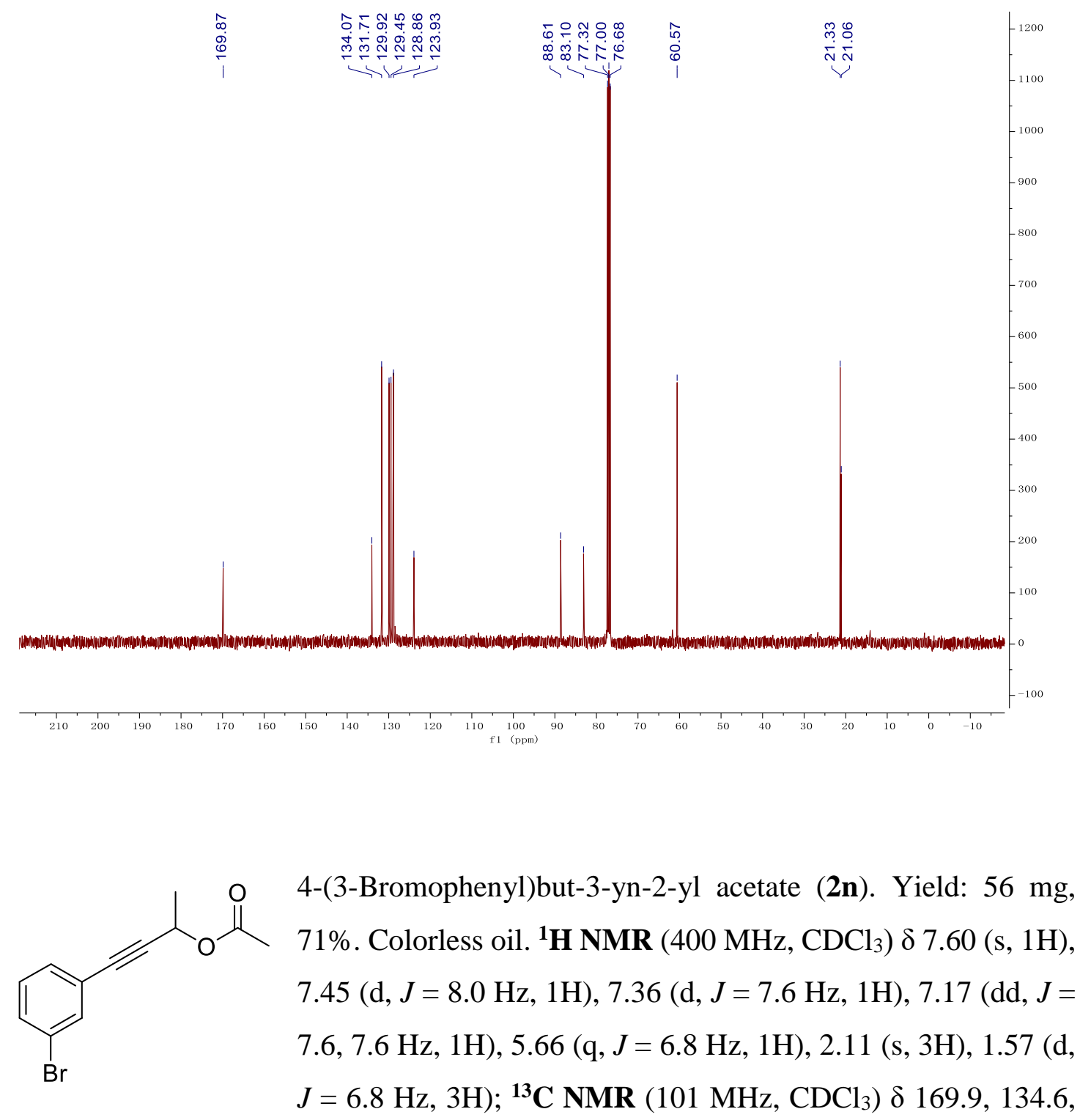

131.7, 130.3, 129.7, 124.2, 122.0, 88.7, 83.0, 60.6, 21.3, 21.1. IR (KBr): v 3066, 2989, 2937, 1744, 1449, 1232, 1036, 955, $786 \mathrm{~cm}^{-1}$. HRMS (ESI) calcd for $\left[\mathrm{C}_{12} \mathrm{H}_{11} \mathrm{BrO}_{2}+\mathrm{Na}\right]^{+}$requires 288.9835, found $288.9837\left[\mathrm{M}^{+}+\mathrm{Na}\right]$. 


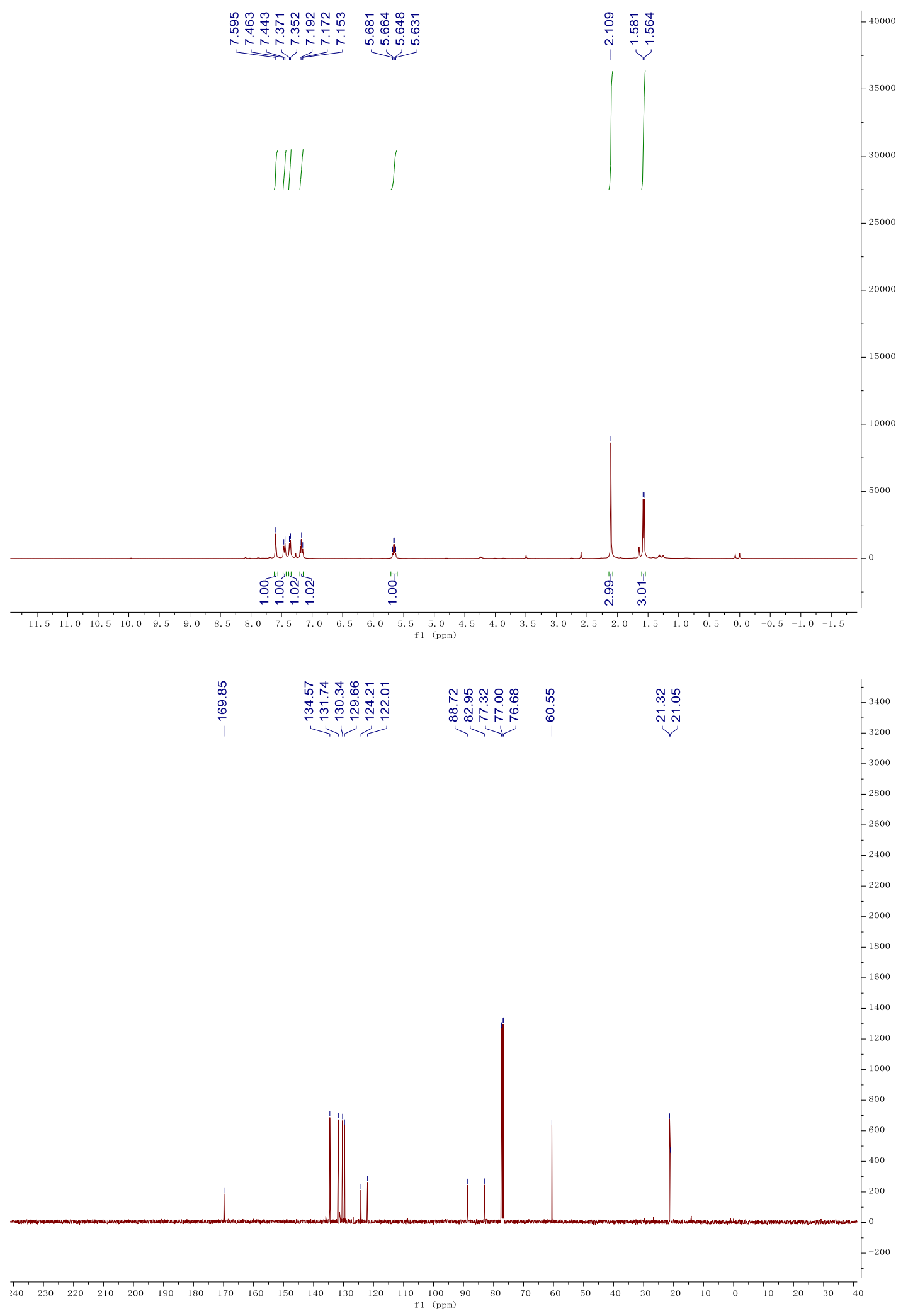




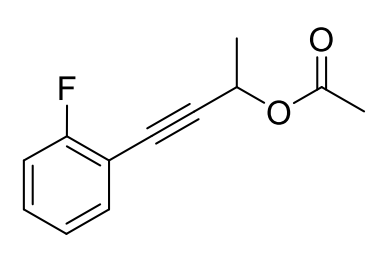

4-(2-Fluorophenyl)but-3-yn-2-yl acetate (2o). Yield: $36 \mathrm{mg}$, 61\%. Colorless oil. ${ }^{\mathbf{1}} \mathbf{H}$ NMR $\left(400 \mathrm{MHz}, \mathrm{CDCl}_{3}\right) \delta 7.43(\mathrm{dd}, J$ = 7.2, 7.2 Hz, 1H), 7.33-7.26 (m, 1H), 7.11-7.03 (m, 2H), 5.71 $(\mathrm{q}, J=6.8 \mathrm{~Hz}, 1 \mathrm{H}), 2.11(\mathrm{~s}, 3 \mathrm{H}), 1.60(\mathrm{~d}, J=6.8 \mathrm{~Hz}, 3 \mathrm{H}) ;{ }^{13} \mathrm{C}$ NMR $\left(101 \mathrm{MHz}, \mathrm{CDCl}_{3}\right) \delta 170.0,162.8\left(J_{\mathrm{CF}}=252.5 \mathrm{~Hz}\right), 133.7,130.4\left(J_{\mathrm{CF}}=32.0 \mathrm{~Hz}\right)$, $123.8\left(J_{\mathrm{CF}}=4.0 \mathrm{~Hz}\right), 115.5\left(J_{\mathrm{CF}}=21.2 \mathrm{~Hz}\right), 110.8\left(J_{\mathrm{CF}}=15.2 \mathrm{~Hz}\right), 92.5\left(J_{\mathrm{CF}}=4.0 \mathrm{~Hz}\right)$, 78.0, 60.7, 21.3, 21.1; ${ }^{19} \mathbf{F}$ NMR $\left(376 \mathrm{MHz}, \mathrm{CDCl}_{3}\right) \delta-109.81--109.87$ (m). IR (KBr): $v$ 3070, 2992, 2939, 1743, 1493, 1235, 10348, 953, $760 \mathrm{~cm}^{-1}$. HRMS (ESI) calcd for $\left[\mathrm{C}_{12} \mathrm{H}_{11} \mathrm{FO}_{2}+\mathrm{Na}\right]^{+}$requires 229.0635, found $229.0637\left[\mathrm{M}^{+}+\mathrm{Na}\right]$.

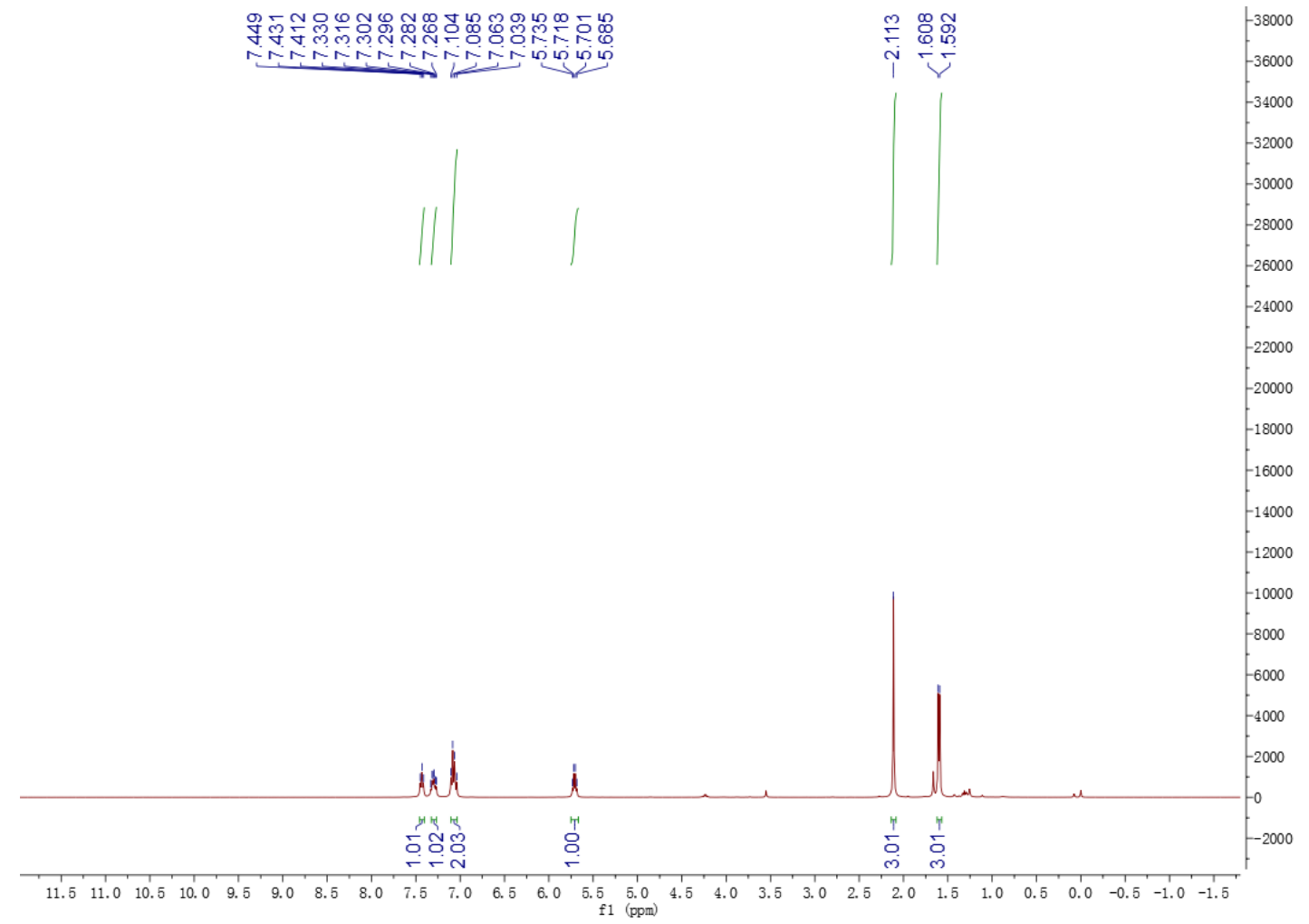




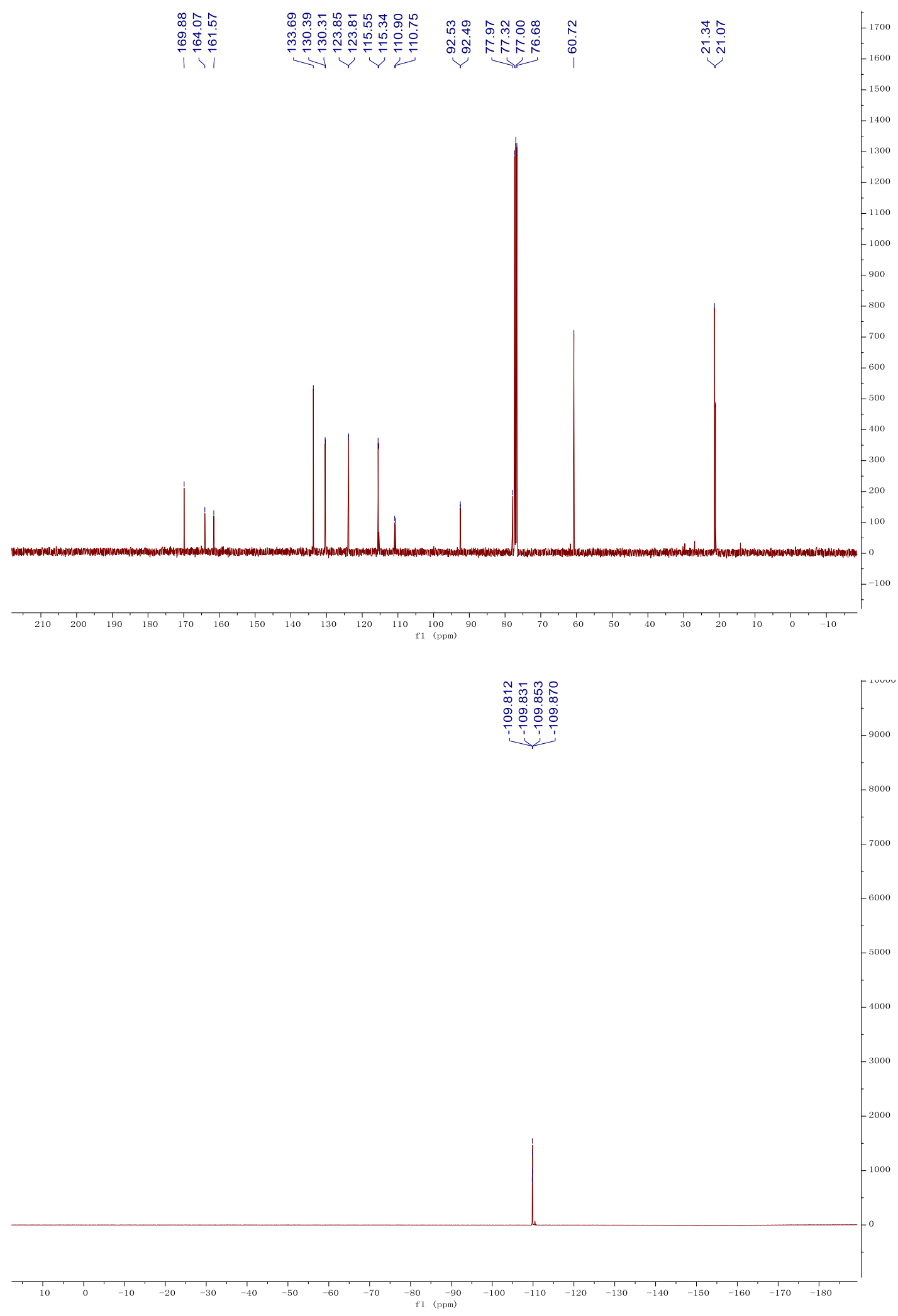


4-(Naphthalen-1-yl)but-3-yn-2-yl acetate (2p). Yield: $51 \mathrm{mg}, 73 \%$. Yellow oil. ${ }^{1} \mathbf{H}$ NMR $\left(400 \mathrm{MHz}, \mathrm{CDCl}_{3}\right) \delta 8.29(\mathrm{~d}, J=8.4 \mathrm{~Hz}, 1 \mathrm{H})$, $7.83(\mathrm{dd}, J=8.4,3.6 \mathrm{~Hz}, 2 \mathrm{H}), 7.68(\mathrm{~d}, J=6.8 \mathrm{~Hz}, 1 \mathrm{H}), 7.58(\mathrm{dd}, J=$

$6.8,6.8 \mathrm{~Hz}, 1 \mathrm{H}), 7.51(\mathrm{dd}, J=6.8,6.8 \mathrm{~Hz}, 1 \mathrm{H}), 7.41(\mathrm{dd}, J=7.6,7.6 \mathrm{~Hz}, 1 \mathrm{H}), 5.82(\mathrm{q}$, $J=6.4 \mathrm{~Hz}, 1 \mathrm{H}), 2.14(\mathrm{~s}, 3 \mathrm{H}), 1.69(\mathrm{~d}, J=6.4 \mathrm{~Hz}, 3 \mathrm{H}) ;{ }^{13} \mathrm{C} \mathrm{NMR}\left(101 \mathrm{MHz}, \mathrm{CDCl}_{3}\right) \delta$ 170.0, 133.3, 133.1, 130.8, 129.1, 128.2, 126.9, 126.4, 126.0, 125.1, 119.8, 92.3, 82.7, 61.0, 21.6, 21.2. IR (KBr): v 3058, 2990, 2936, 1740, 1447, 1233, 1022, 950, $775 \mathrm{~cm}^{-}$ 1. HRMS (ESI) calcd for $\left[\mathrm{C}_{16} \mathrm{H}_{14} \mathrm{O}_{2}+\mathrm{H}\right]^{+}$requires 239.1067, found $239.1069\left[\mathrm{M}^{+}+\mathrm{H}\right]$.

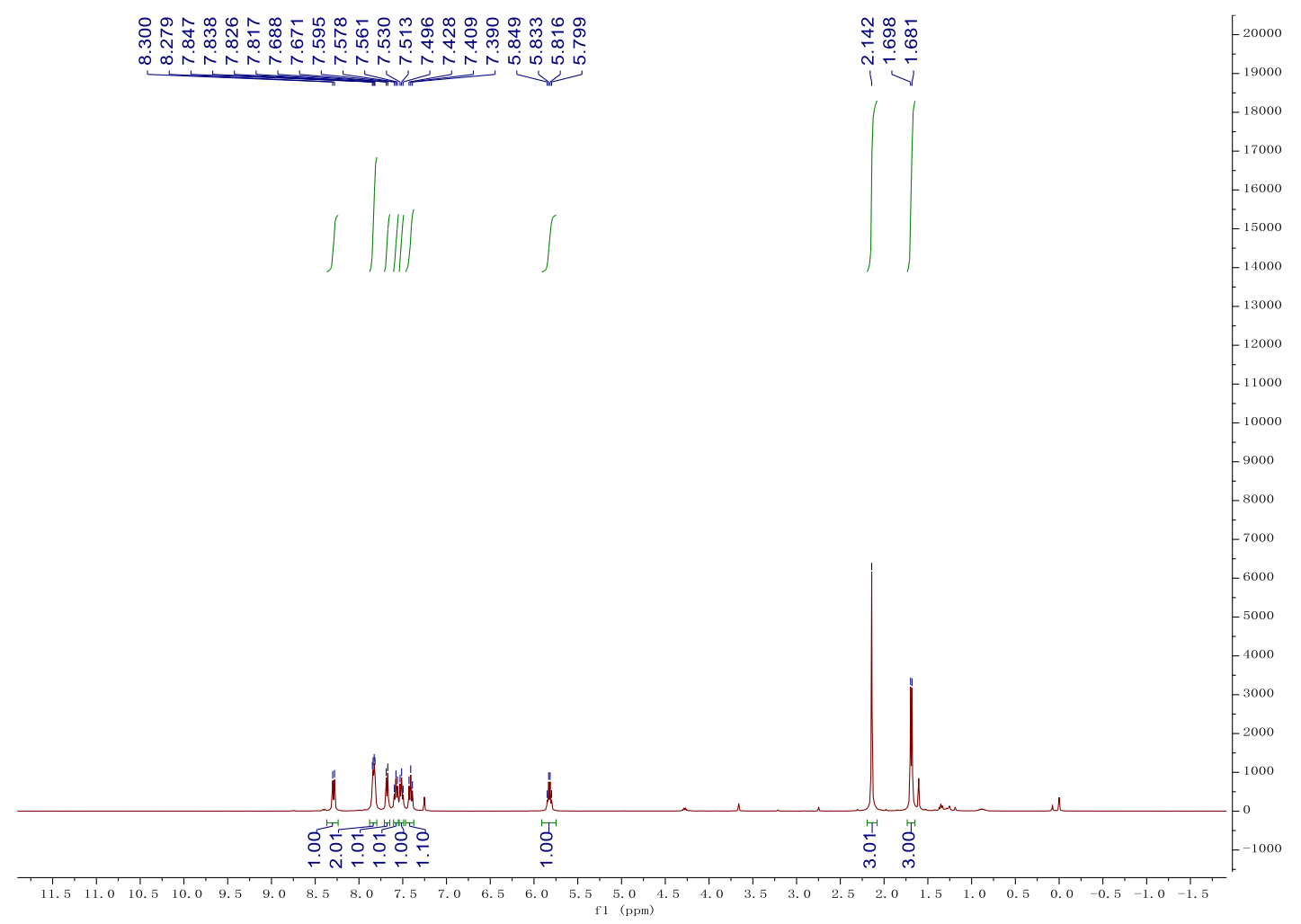




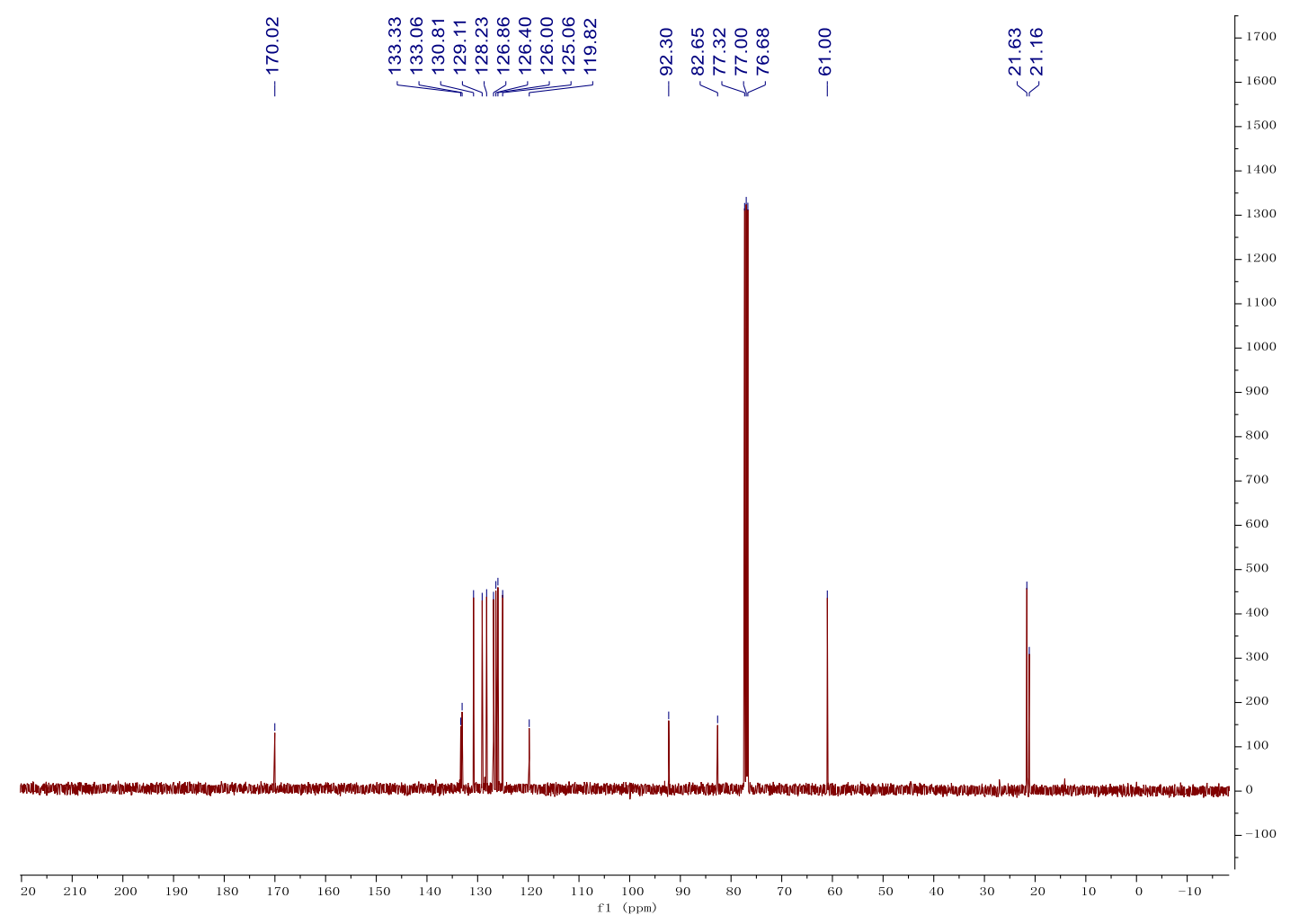

4-(Thiophen-2-yl)but-3-yn-2-yl acetate $(\mathbf{2 q}) . A$ A known
Compound $^{[4]}$. Yield: $29 \mathrm{mg}, 50 \%$. Colorless oil. ${ }^{\mathbf{1}} \mathbf{H}$ NMR $(400$ 21.3, 21.1. 

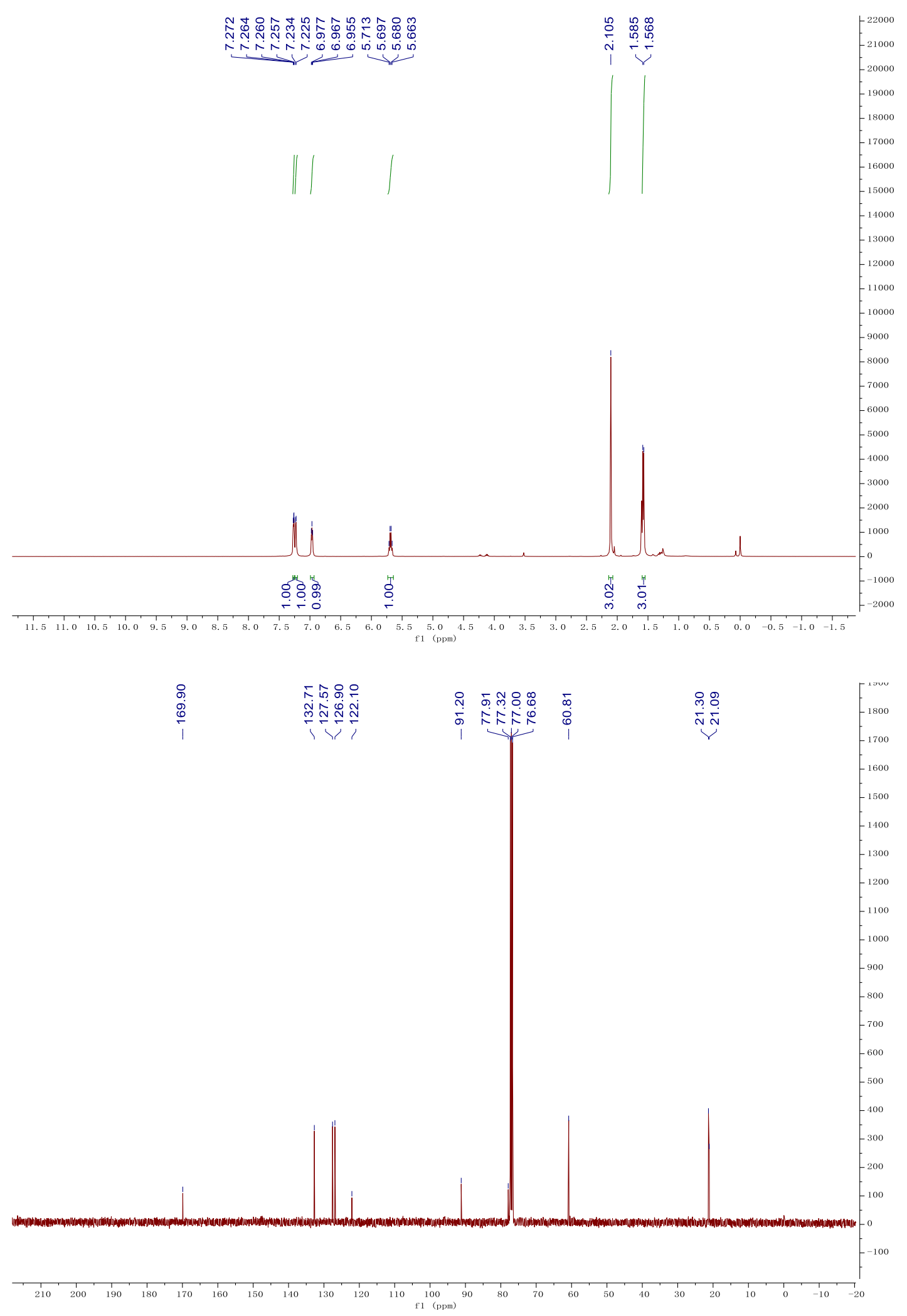

4-(Thiophen-3-yl)but-3-yn-2-yl acetate (2r) Yield: $33 \mathrm{mg}, 57 \%$. Colorless oil. ${ }^{\mathbf{1}} \mathbf{H} \mathbf{N M R}\left(400 \mathrm{MHz}, \mathrm{CDCl}_{3}\right) \delta 7.47(\mathrm{~d}, J=1.6 \mathrm{~Hz}$,
$\mathrm{1H}), 7.26-7.24(\mathrm{~m}, 1 \mathrm{H}), 7.11(\mathrm{~d}, J=4.8 \mathrm{~Hz}, 1 \mathrm{H}), 5.66(\mathrm{q}, J=6.8$
$\mathrm{Hz}, 1 \mathrm{H}), 2.10(\mathrm{~s}, 3 \mathrm{H}), 1.57(\mathrm{~d}, J=6.8 \mathrm{~Hz}, 3 \mathrm{H}) ;{ }^{\mathbf{1 3}} \mathbf{C} \mathbf{N M R}(101$ 
$\left.\mathrm{MHz}, \mathrm{CDCl}_{3}\right) \delta 169.9,129.9,129.5,125.3,121.2,87.0,79.7,60.8,21.5,21.1 . \mathbf{I R}(\mathrm{KBr})$ : v 3110, 2990, 2937, 1742, 1370, 1233, 1036, 966, $787 \mathrm{~cm}^{-1}$. HRMS (ESI) calcd for $\left[\mathrm{C}_{10} \mathrm{H}_{10} \mathrm{O}_{2} \mathrm{~S}+\mathrm{H}\right]^{+}$requires 195.0474 , found $195.0476\left[\mathrm{M}^{+}+\mathrm{H}\right]$.

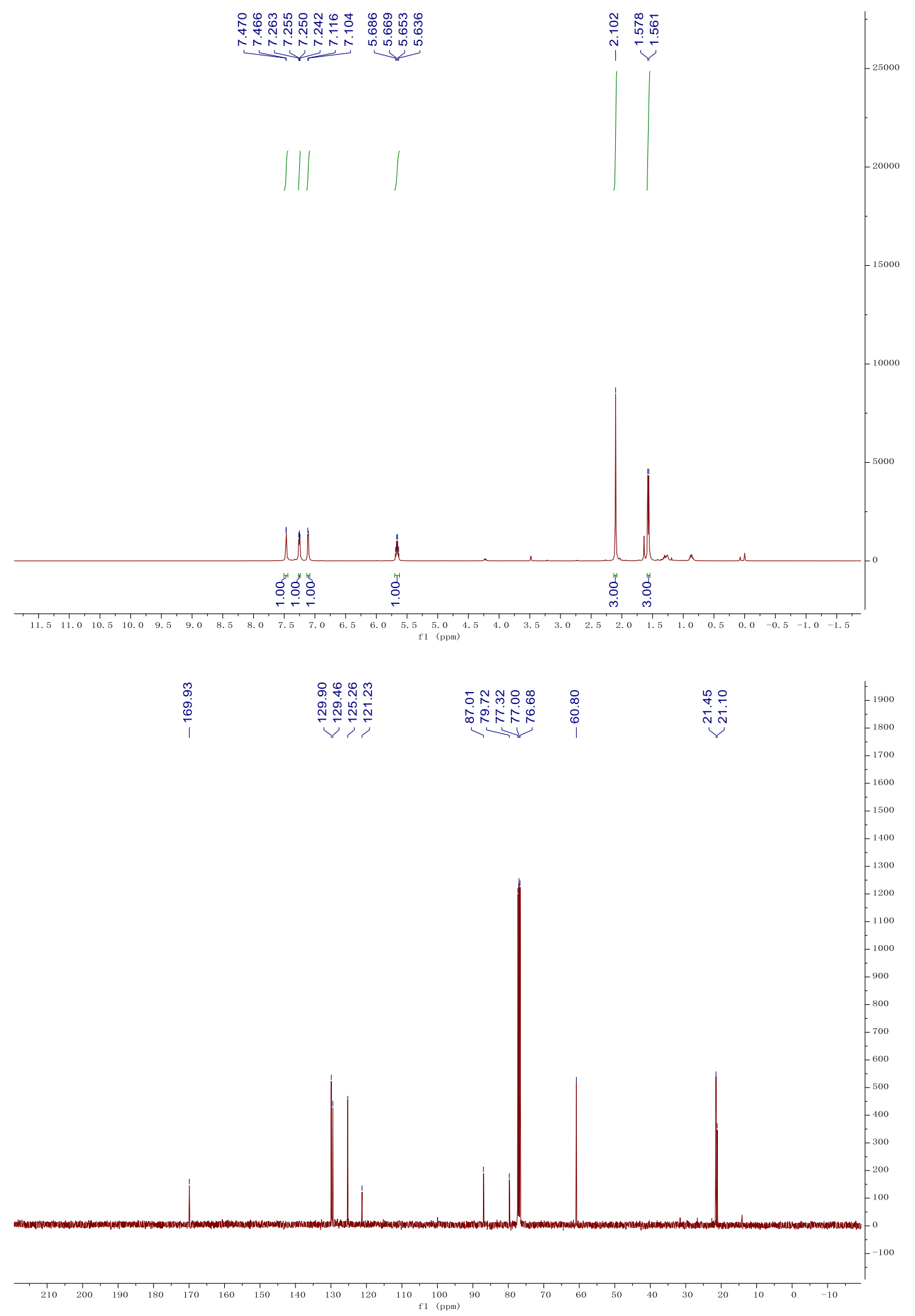




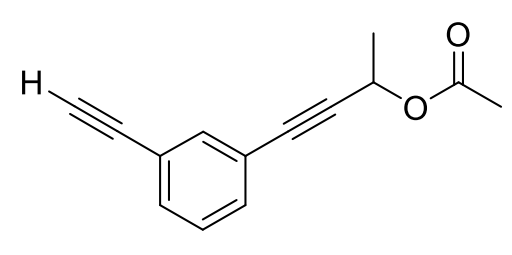

4-(3-Ethynylphenyl)but-3-yn-2-yl acetate (2s). Yield: $29 \mathrm{mg}, 45 \%$. Colorless oil. ${ }^{\mathbf{1}} \mathbf{H}$ NMR (400 MHz, $\left.\mathrm{CDCl}_{3}\right) \delta 7.58(\mathrm{~s}, 1 \mathrm{H}), 7.43(\mathrm{dd}, J=8.6,8.6 \mathrm{~Hz}, 2 \mathrm{H})$, $7.27(\mathrm{dd}, J=7.2,7.2 \mathrm{~Hz}, 1 \mathrm{H}), 5.66(\mathrm{q}, J=6.8 \mathrm{~Hz}, 1 \mathrm{H})$, $3.01(\mathrm{~s}, 1 \mathrm{H}), 2.11(\mathrm{~s}, 3 \mathrm{H}), 1.58(\mathrm{~d}, J=6.8 \mathrm{~Hz}, 3 \mathrm{H}) ;{ }^{13} \mathrm{C} \mathrm{NMR}\left(101 \mathrm{MHz}, \mathrm{CDCl}_{3}\right) \delta$ 169.9, 135.4, 132.1, 132.1, 128.3, 122.6, 122.4, 88.1, 83.5, 82.6, 77.8, 60.7, 21.4, 21.1. IR (KBr): v 3065, 2990, 2935, 1744, 1441, 1235, 1037, 963, 798. HRMS (ESI) calcd for $\left[\mathrm{C}_{14} \mathrm{H}_{12} \mathrm{O}_{2}+\mathrm{Na}\right]^{+}$requires 235.0730 , found $235.0732\left[\mathrm{M}^{+}+\mathrm{Na}\right]$

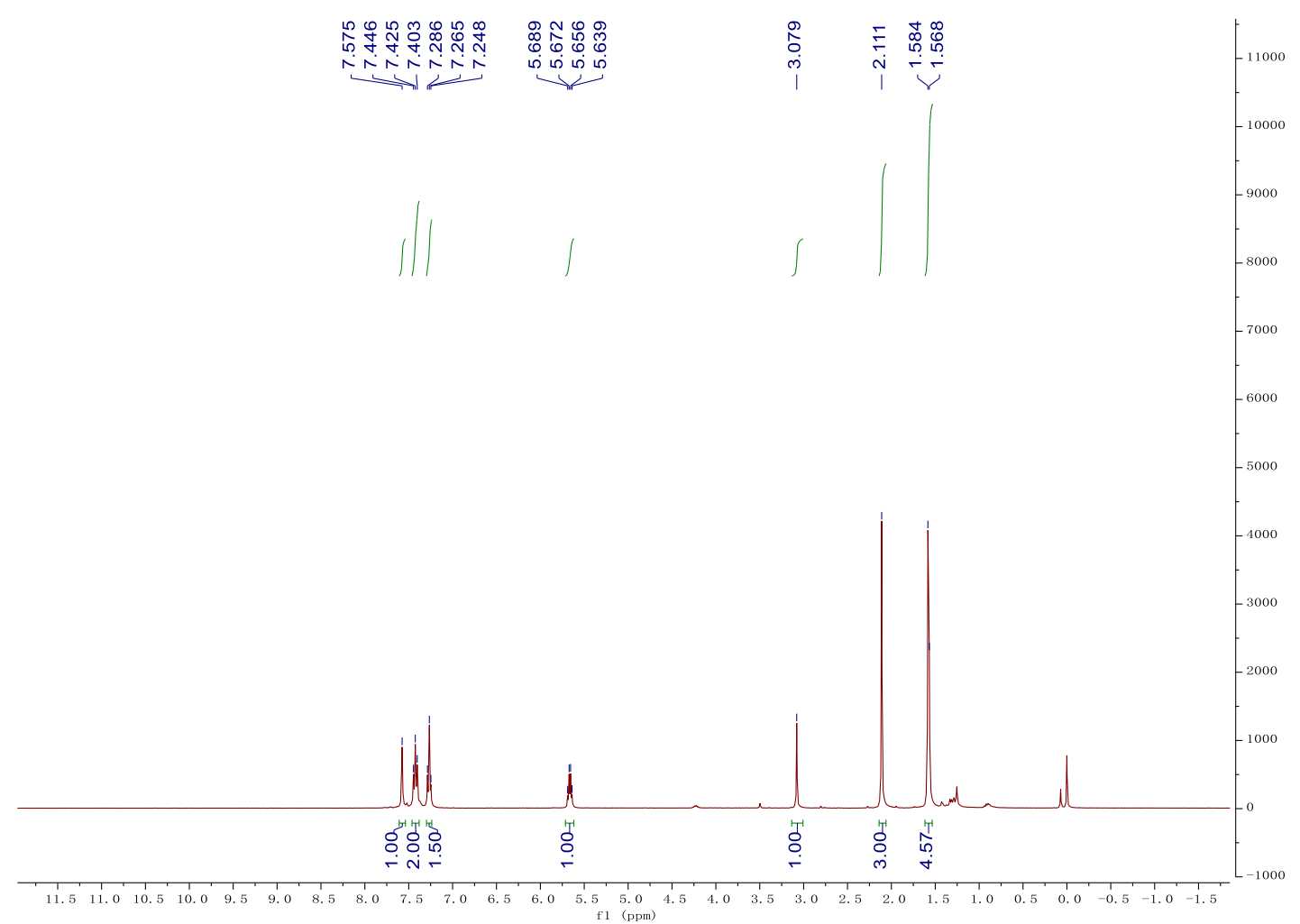




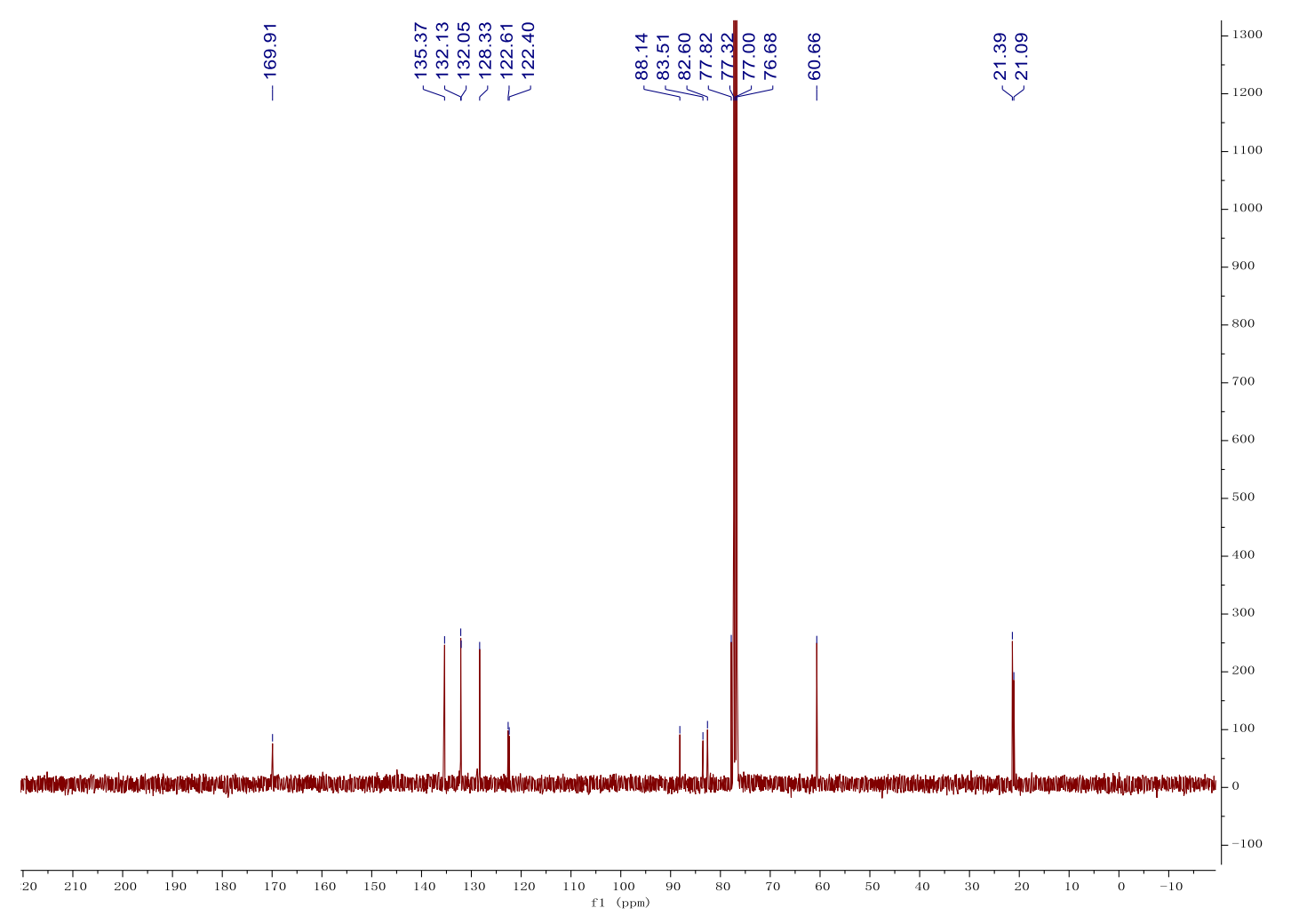

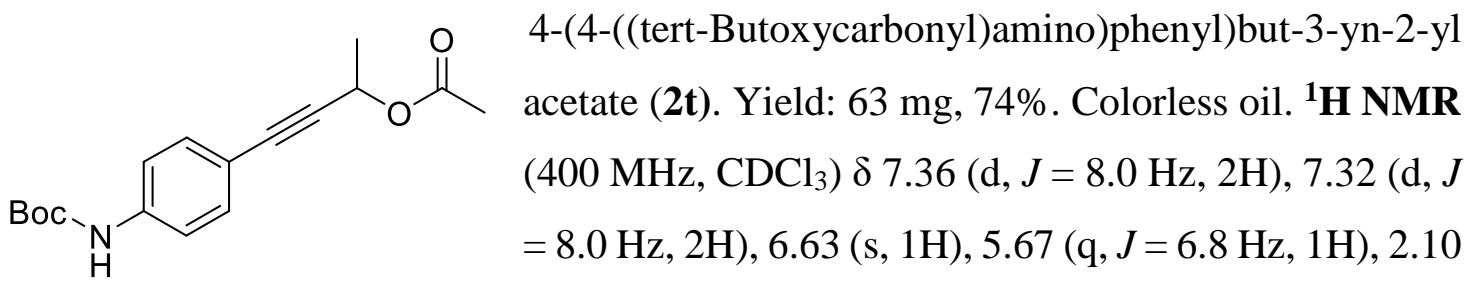
(s, 3H), 1.67 (d, $J=6.8 \mathrm{~Hz}, 3 \mathrm{H}), 1.51(\mathrm{~s}, 9 \mathrm{H}) ;{ }^{13} \mathrm{C} \mathrm{NMR}\left(101 \mathrm{MHz}, \mathrm{CDCl}_{3}\right) \delta 170.0$, $152.3,138.8,132.7,117.8,116.4,86.5,84.4,80.8,60.9,28.3,21.5,21.1$. IR (KBr): v 3341, 3099, 2981, 2935, 1728, 1523, 1232, 1033, 953, 839. HRMS (ESI) calcd for $\left[\mathrm{C}_{17} \mathrm{H}_{21} \mathrm{NO}_{4}+\mathrm{Na}\right]^{+}$requires 326.1363 , found $326.1365\left[\mathrm{M}^{+}+\mathrm{Na}\right]$. 


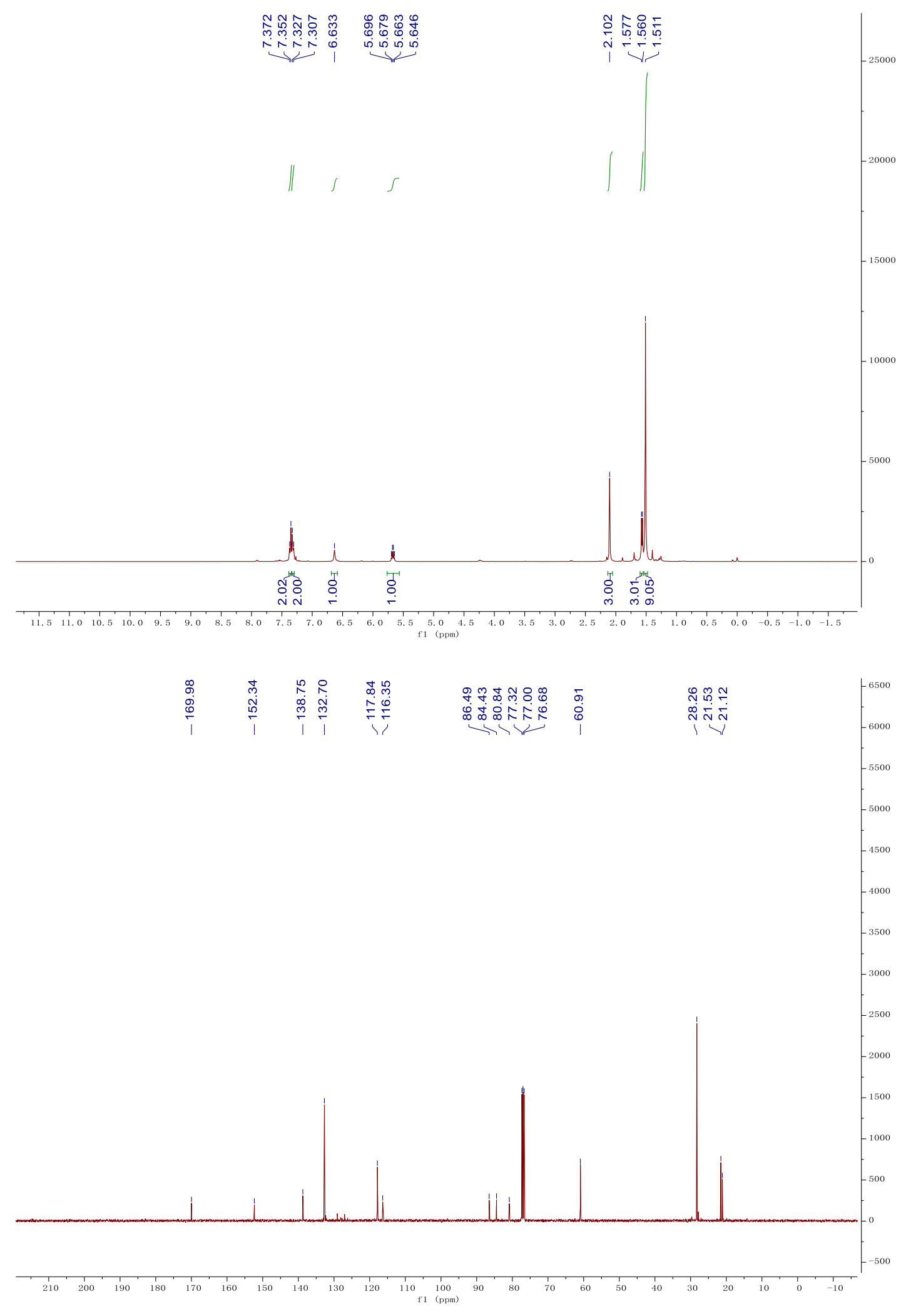




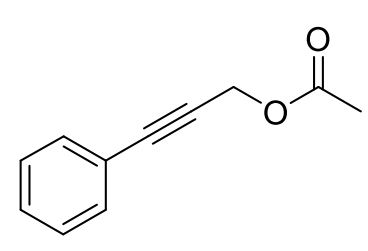

3-Phenylprop-2-yn-1-yl acetate (3a). A known compound ${ }^{[5]}$. Yield: $34 \mathrm{mg}, 66 \%$. Colorless oil. ${ }^{1} \mathbf{H}$ NMR $\left(400 \mathrm{MHz}, \mathrm{CDCl}_{3}\right)$ $\delta$ 7.47-7.44 (m, 2H), 7.33-7.31 (m, 3H), $4.91(\mathrm{~s}, 2 \mathrm{H}), 2.14(\mathrm{~s}$, $3 \mathrm{H}) ;{ }^{13} \mathbf{C}$ NMR $\left(101 \mathrm{MHz}, \mathrm{CDCl}_{3}\right) \delta 170.3,131.9,128.8,128.3$, $122.1,86.4,82.9,52.8,20.8$.

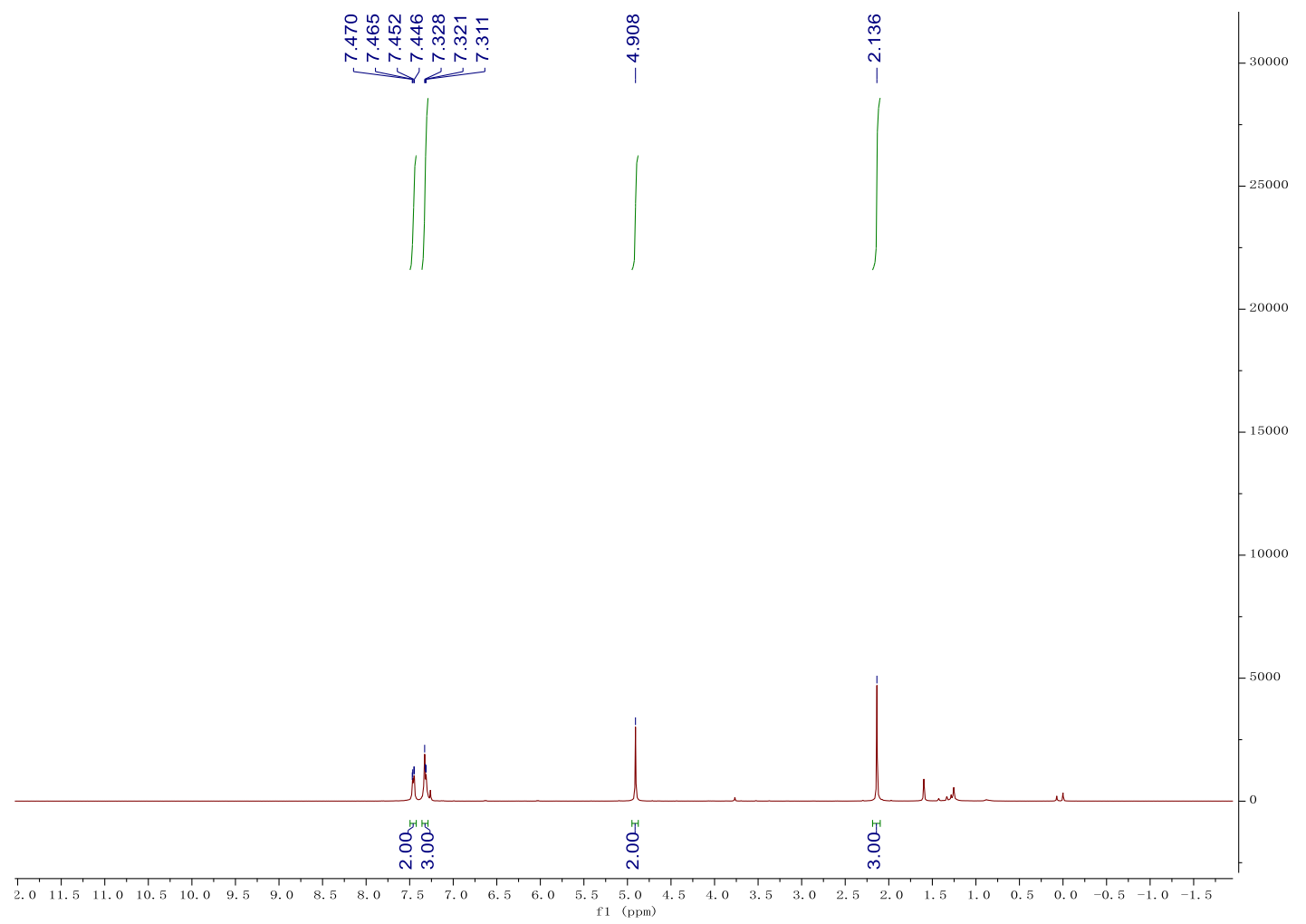



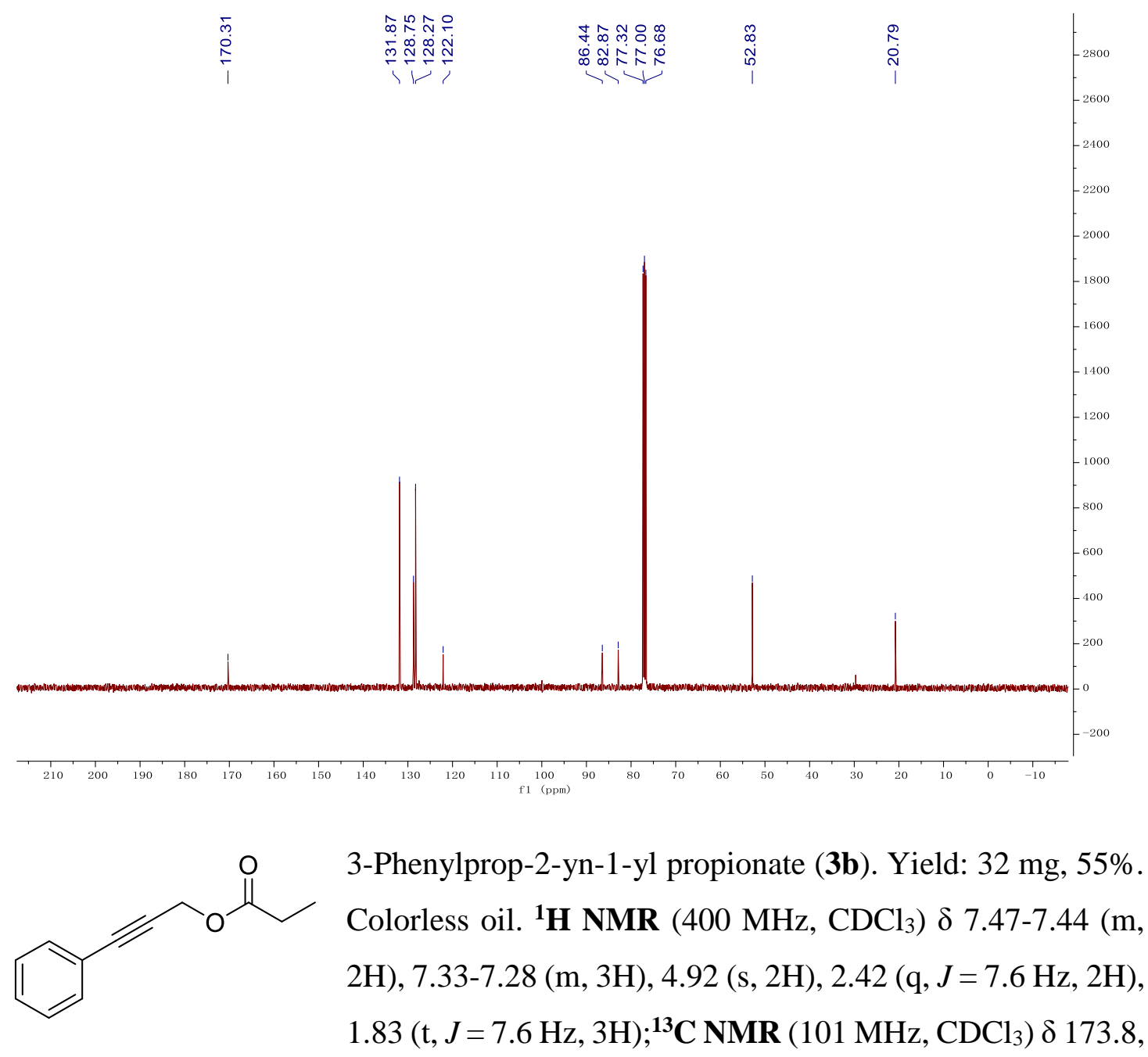

131.9, 128.7, 128.3, 122.2, 86.4, 83.0, 52.7, 27.4, 8.99. IR (KBr): v 3056, 2983, 2939, 1745, 1491, 1344, 1174, 1032, $759 \mathrm{~cm}^{-1}$. HRMS (ESI) calcd for $\left[\mathrm{C}_{12} \mathrm{H}_{12} \mathrm{O}_{2}+\mathrm{Na}\right]^{+}$ requires 211.0730, found 211.0731 $\left[\mathrm{M}^{+}+\mathrm{Na}\right]$ 

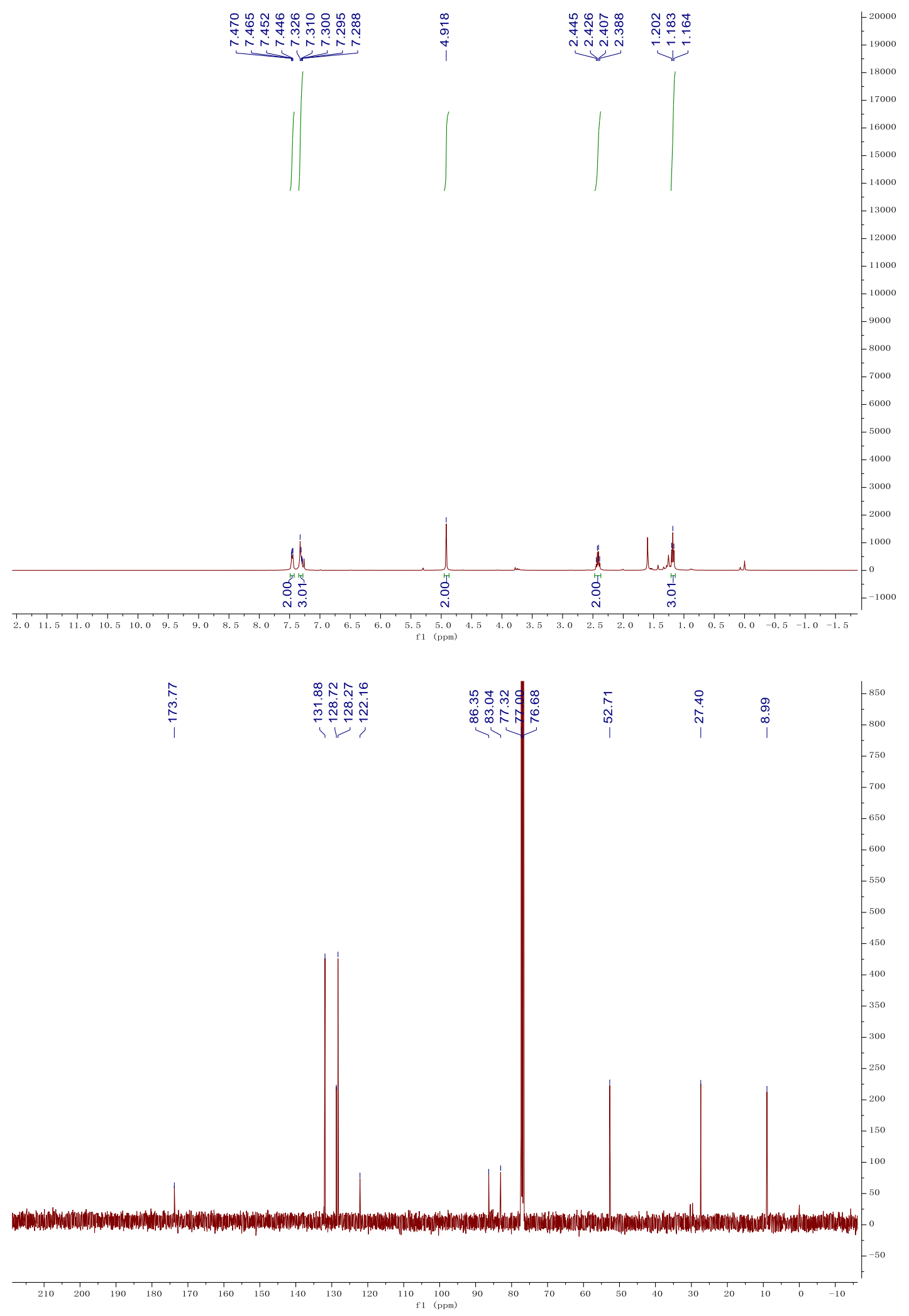


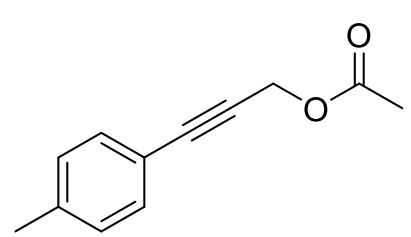

3-(p-Tolyl)prop-2-yn-1-yl acetate (3c). A known compound $^{[6]}$. Yield: $39 \mathrm{mg}, 70 \%$. Colorless oil. ${ }^{\mathbf{1}} \mathbf{H}$ NMR $\left(400 \mathrm{MHz}, \mathrm{CDCl}_{3}\right) \delta 7.35(\mathrm{~d}, J=7.6 \mathrm{~Hz}, 2 \mathrm{H}), 7.12(\mathrm{~d}, J=7.6$ $\mathrm{Hz}, 2 \mathrm{H}), 4.90$ (s, 2H), 2.35 (s, 3H), 2.13 (s, 3H); ${ }^{13} \mathrm{C}$ NMR $\left(101 \mathrm{MHz} \mathrm{CDCl}_{3}\right) \delta 170.3,138.9,131.8,129.0,119.0,86.6,82.2,52.9,21.5,20.8$.

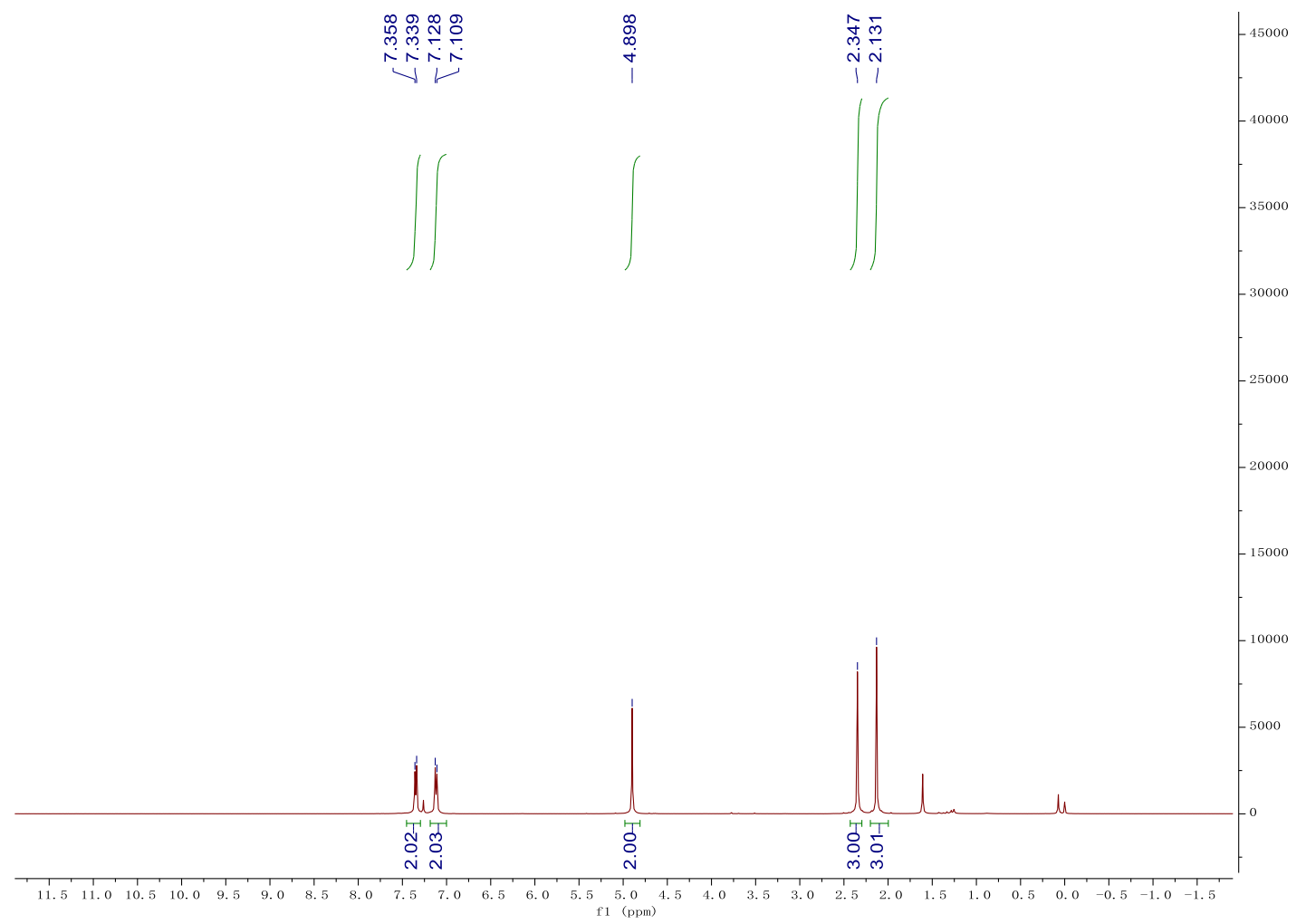



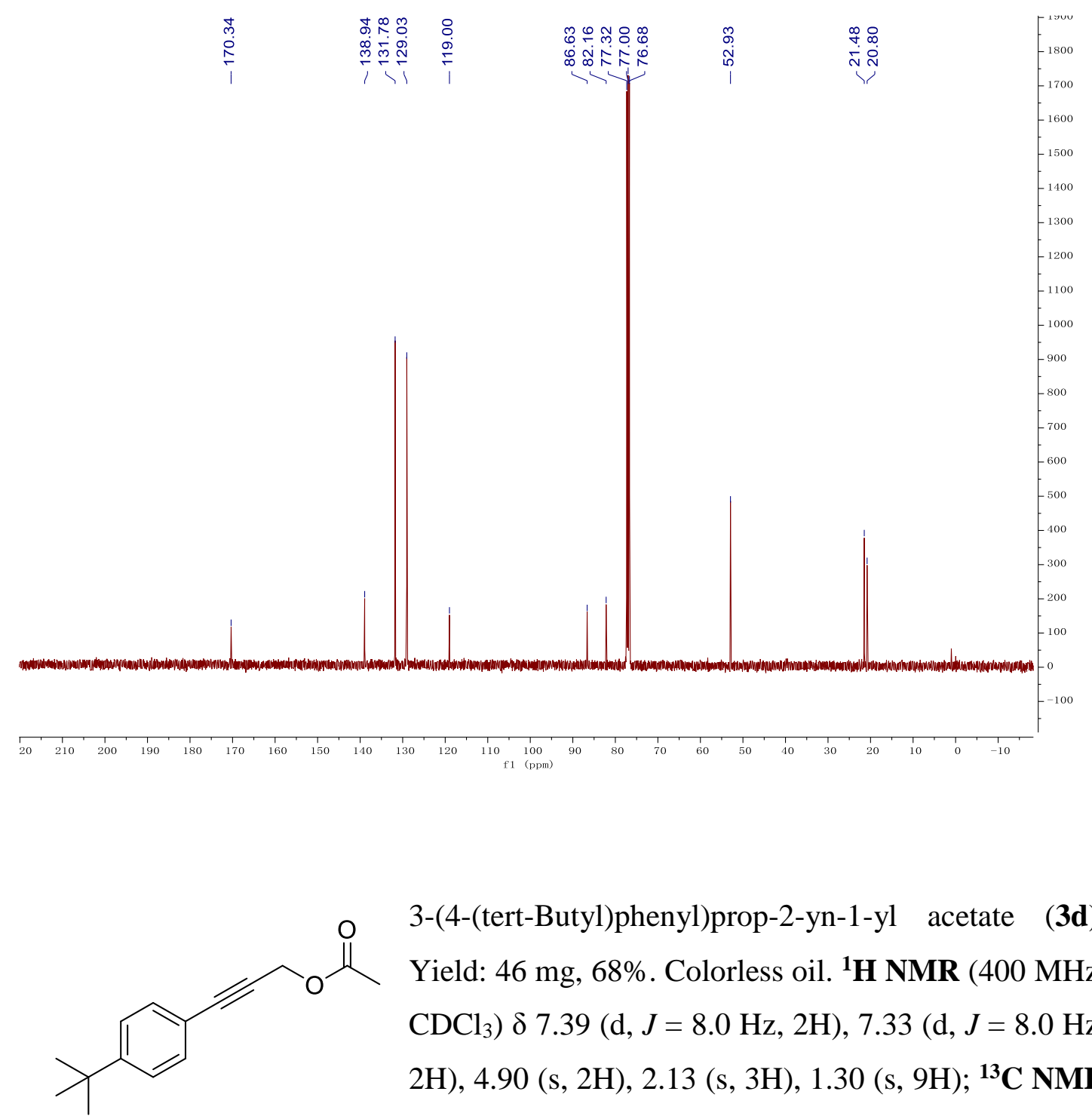

3-(4-(tert-Butyl)phenyl)prop-2-yn-1-yl acetate (3d). Yield: 46 mg, 68\%. Colorless oil. ${ }^{1}$ H NMR (400 MHz, $\left.\mathrm{CDCl}_{3}\right) \delta 7.39(\mathrm{~d}, J=8.0 \mathrm{~Hz}, 2 \mathrm{H}), 7.33(\mathrm{~d}, J=8.0 \mathrm{~Hz}$, 2H), 4.90 (s, 2H), 2.13 (s, 3H), 1.30 (s, 9H); ${ }^{13}$ C NMR $\left(101 \mathrm{MHz}, \mathrm{CDCl}_{3}\right) \delta 170.3,152.1,131.6,125.3,119.1$,

86.6, 82.2, 52.9, 34.8, 31.1, 20.8. IR (KBr): v 3038, 2963, 2870, 1747, 1361, 1224, $1039,955,836 \mathrm{~cm}^{-1}$. HRMS (ESI) calcd for $\left[\mathrm{C}_{15} \mathrm{H}_{18} \mathrm{O}_{2}+\mathrm{Na}\right]^{+}$requires 253.1199 , found $253.1199\left[\mathrm{M}^{+}+\mathrm{Na}\right]$ 

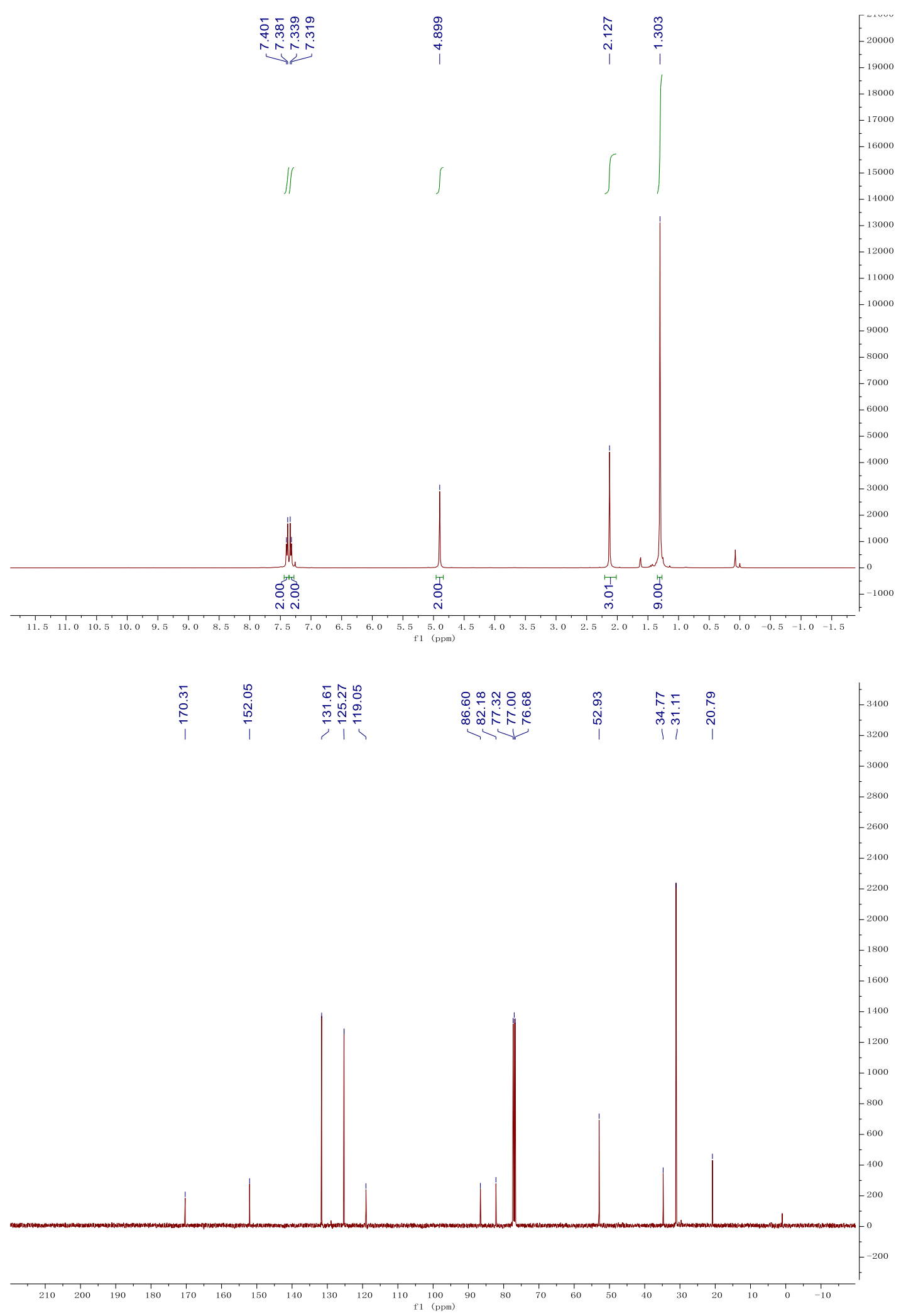


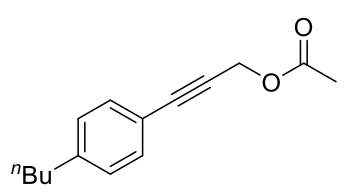

3-(4-Butylphenyl)prop-2-yn-1-yl acetate (3e). Yield: $48 \mathrm{mg}, 71 \%$.

Colorless oil. ${ }^{1} \mathbf{H}$ NMR $\left(400 \mathrm{MHz}, \mathrm{CDCl}_{3}\right) \delta 7.36(\mathrm{~d}, J=7.6 \mathrm{~Hz}$, $2 \mathrm{H}), 7.12(\mathrm{~d}, J=7.6 \mathrm{~Hz}, 2 \mathrm{H}), 4.90(\mathrm{~s}, 2 \mathrm{H}), 2.60(\mathrm{t}, J=7.6 \mathrm{~Hz}$, 2H), $2.13(\mathrm{~s}, 3 \mathrm{H}), 1.62-1.53(\mathrm{~m}, 2 \mathrm{H}), 1.37-1.28(\mathrm{~m}, 2 \mathrm{H}), 0.92(\mathrm{t}, J=7.2 \mathrm{~Hz}, 3 \mathrm{H}) ;{ }^{13} \mathrm{C}$ NMR $\left(101 \mathrm{MHz}, \mathrm{CDCl}_{3}\right) \delta 170.3,143.9,131.8,128.4,119.2,86.7,82.2,52.9,35.6$, 33.3, 22.3, 20.8, 13.9. IR (KBr): v 3030, 2959, 2931, 1748, 1378, 1224, 1029, 955, 801 $\mathrm{cm}^{-1}$. HRMS (ESI) calcd for $\left[\mathrm{C}_{15} \mathrm{H}_{18} \mathrm{O}_{2}+\mathrm{Na}\right]^{+}$requires 253.1199 , found 253.1199 $\left[\mathrm{M}^{+}+\mathrm{Na}\right]$.

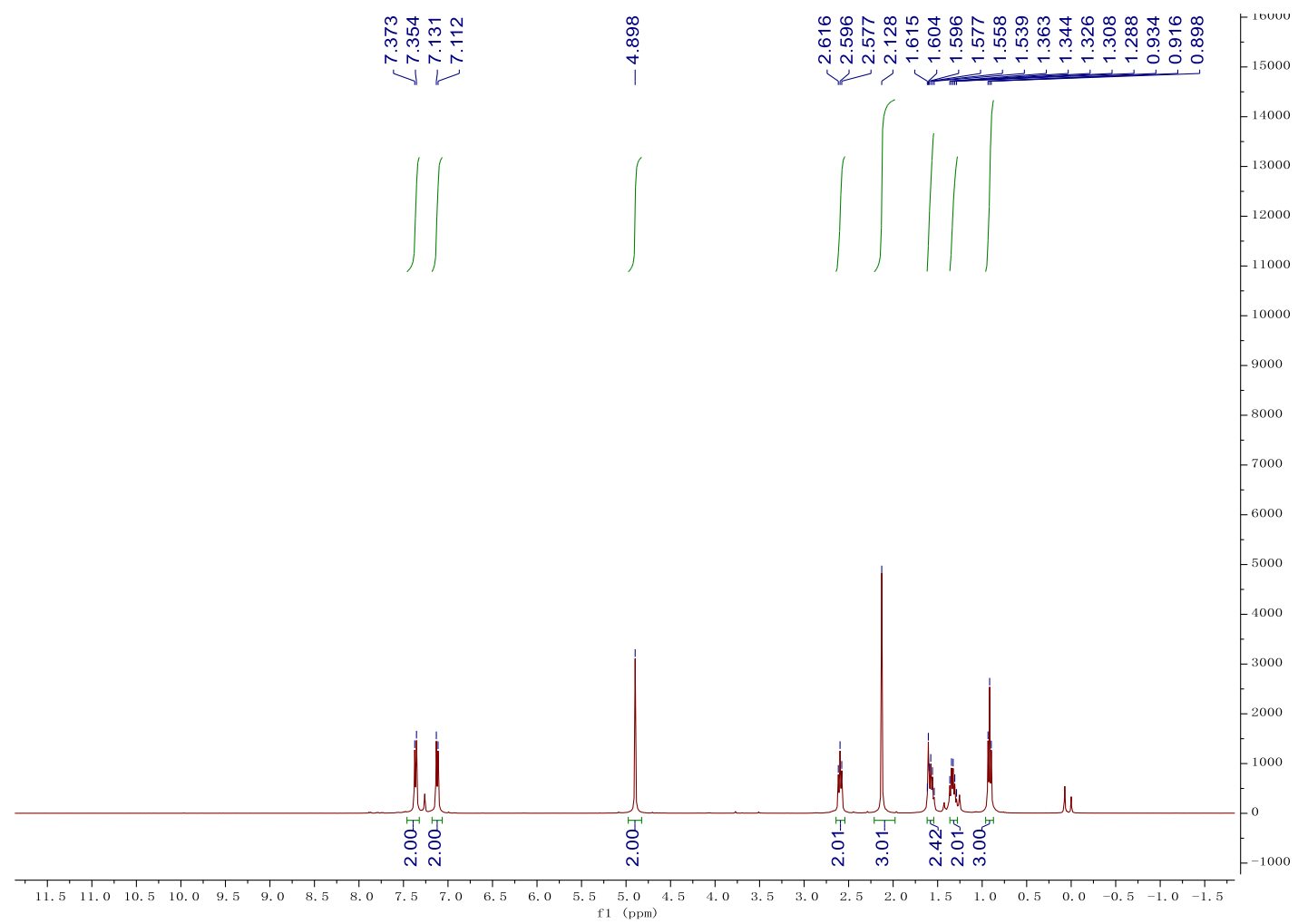




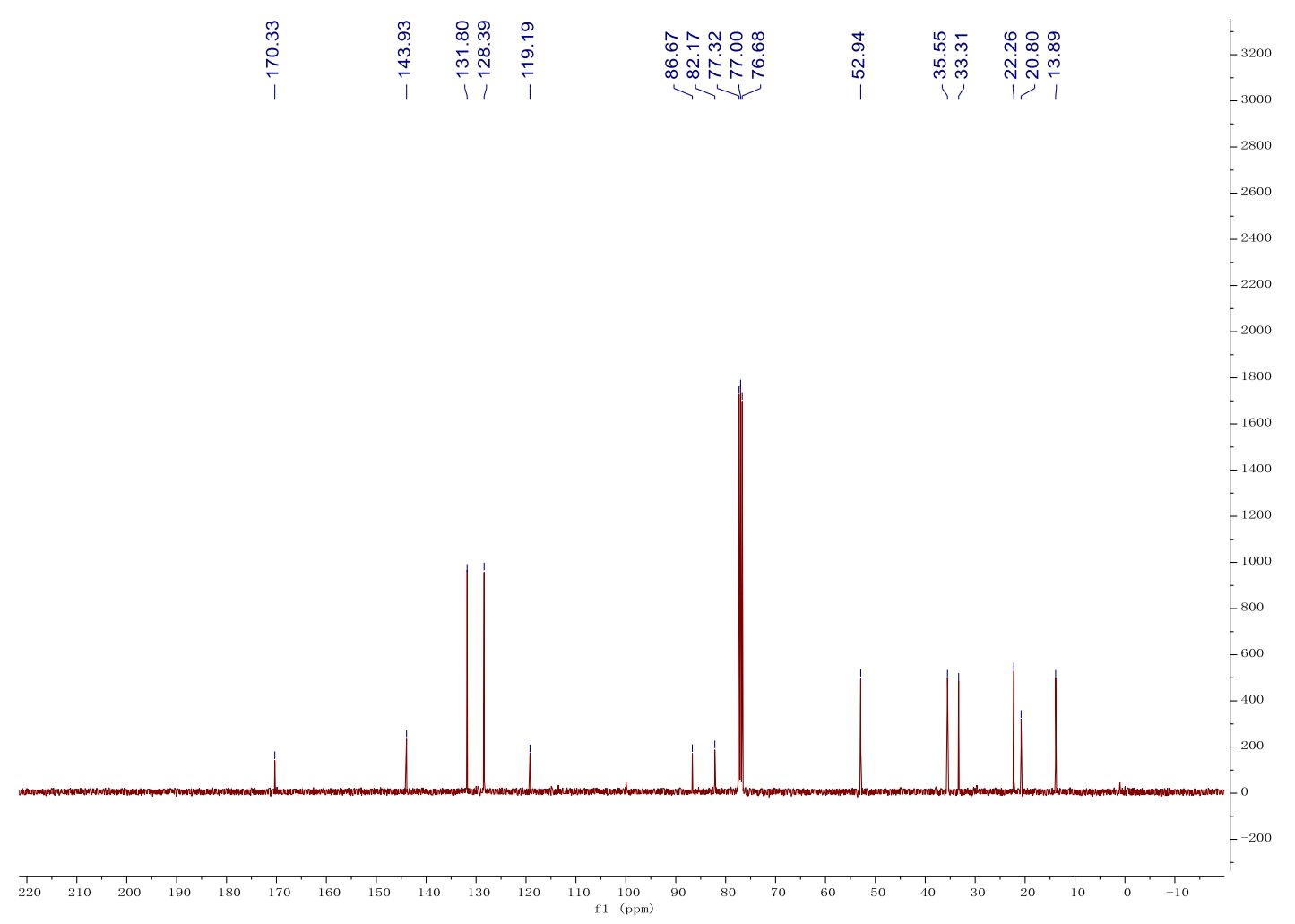

3-(4-Methoxyphenyl)prop-2-yn-1-yl acetate (3f). A known
$\begin{aligned} & \text { compound }{ }^{[7]} \text {. Yield: } 40 \mathrm{mg}, 66 \% \text {. Colorless oil. }{ }^{\mathbf{1}} \mathbf{H} \text { NMR } \\ & 8.4 \mathrm{~Hz}, 2 \mathrm{H}), 4.89(\mathrm{~s}, 2 \mathrm{H}), 3.81(\mathrm{~s}, 3 \mathrm{H}), 2.13(\mathrm{~s}, 3 \mathrm{H}) ;{ }^{\mathbf{1 3}} \mathrm{C}\end{aligned}$

NMR $\left(101 \mathrm{MHz}, \mathrm{CDCl}_{3}\right) \delta 170.4,159.9,133.4,114.1,113.9,86.5,81.5,55.3,53.0$,

20.8 . 

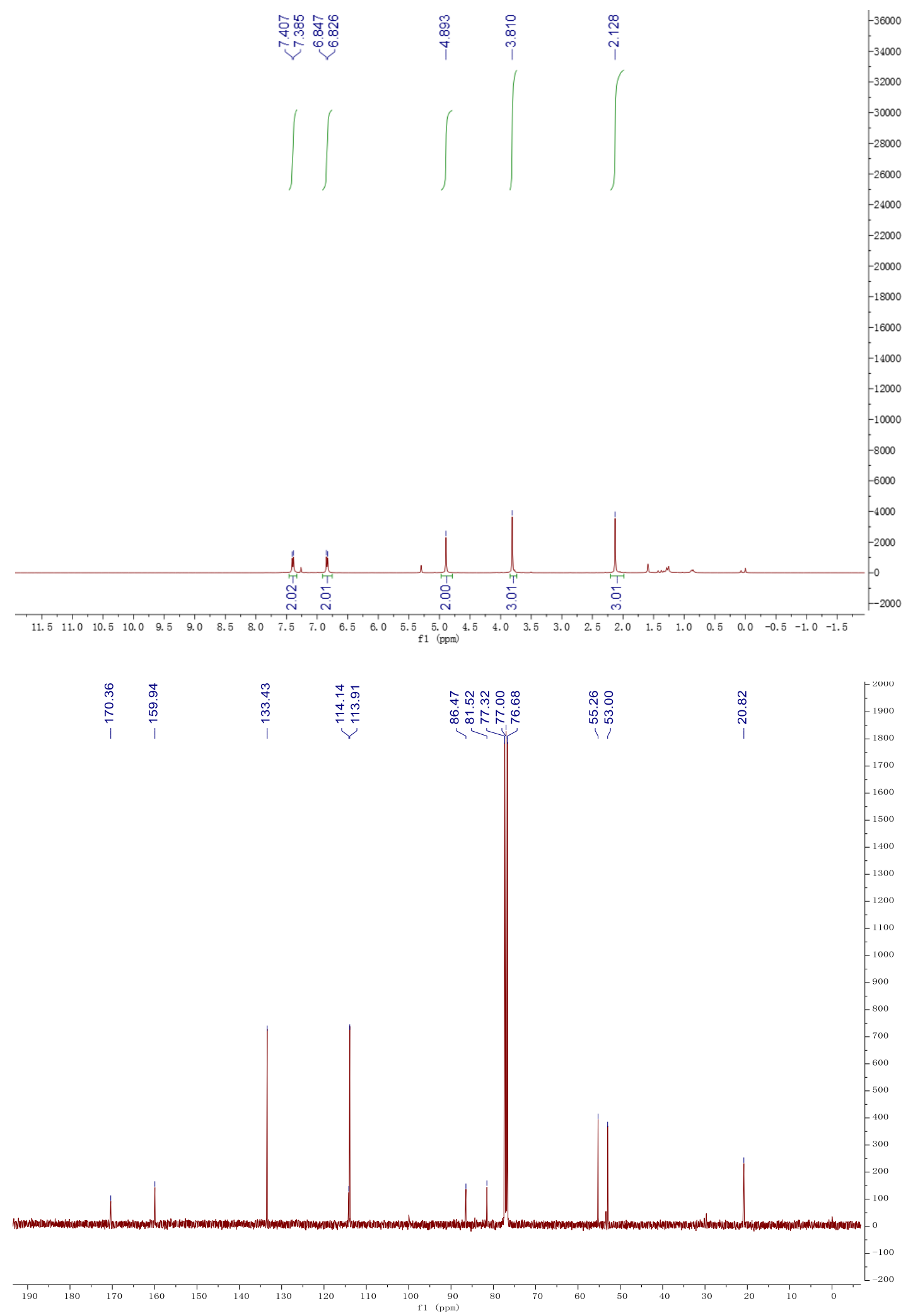


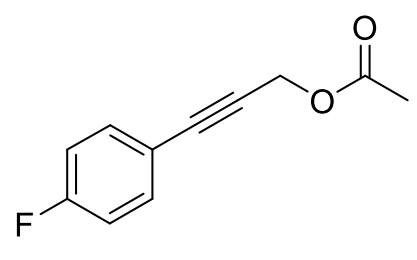

3-(4-Fluorophenyl)prop-2-yn-1-yl acetate. 3g. Yield: 36 mg, $63 \%$. Colorless oil. ${ }^{1} \mathbf{H}$ NMR $\left(400 \mathrm{MHz}, \mathrm{CDCl}_{3}\right) \delta$ 7.46-7.42 (m, 2H), $7.01(\mathrm{dd}, J=8.4,8.4 \mathrm{~Hz}, 2 \mathrm{H}), 4.89$ (s, 2H), 2.14 (s, $3 \mathrm{H}) ;{ }^{13} \mathrm{C}$ NMR $\left(101 \mathrm{MHz}, \mathrm{CDCl}_{3}\right) \delta 170.3,163.0\left(J_{\mathrm{CF}}=\right.$ $252.5), 133.8\left(J_{\mathrm{CF}}=8.1\right), 118.2\left(J_{\mathrm{CF}}=4.04\right), 115.6\left(J_{\mathrm{CF}}=22.2\right), 85.4,82.7,52.7,20.8$; ${ }^{19} \mathrm{~F}$ NMR $\left(376 \mathrm{MHz}, \mathrm{CDCl}_{3}\right) \delta-110.06--110.13$ (m, 1F). IR (KBr): v 3074, 2941, $2855,1747,1508,1224,1030,955,838 \mathrm{~cm}^{-1}$. HRMS (ESI) calcd for $\left[\mathrm{C}_{11} \mathrm{H}_{9} \mathrm{FO}_{2}+\mathrm{Na}\right]^{+}$ requires 215.0479 , found $215.0480\left[\mathrm{M}^{+}+\mathrm{Na}\right]$

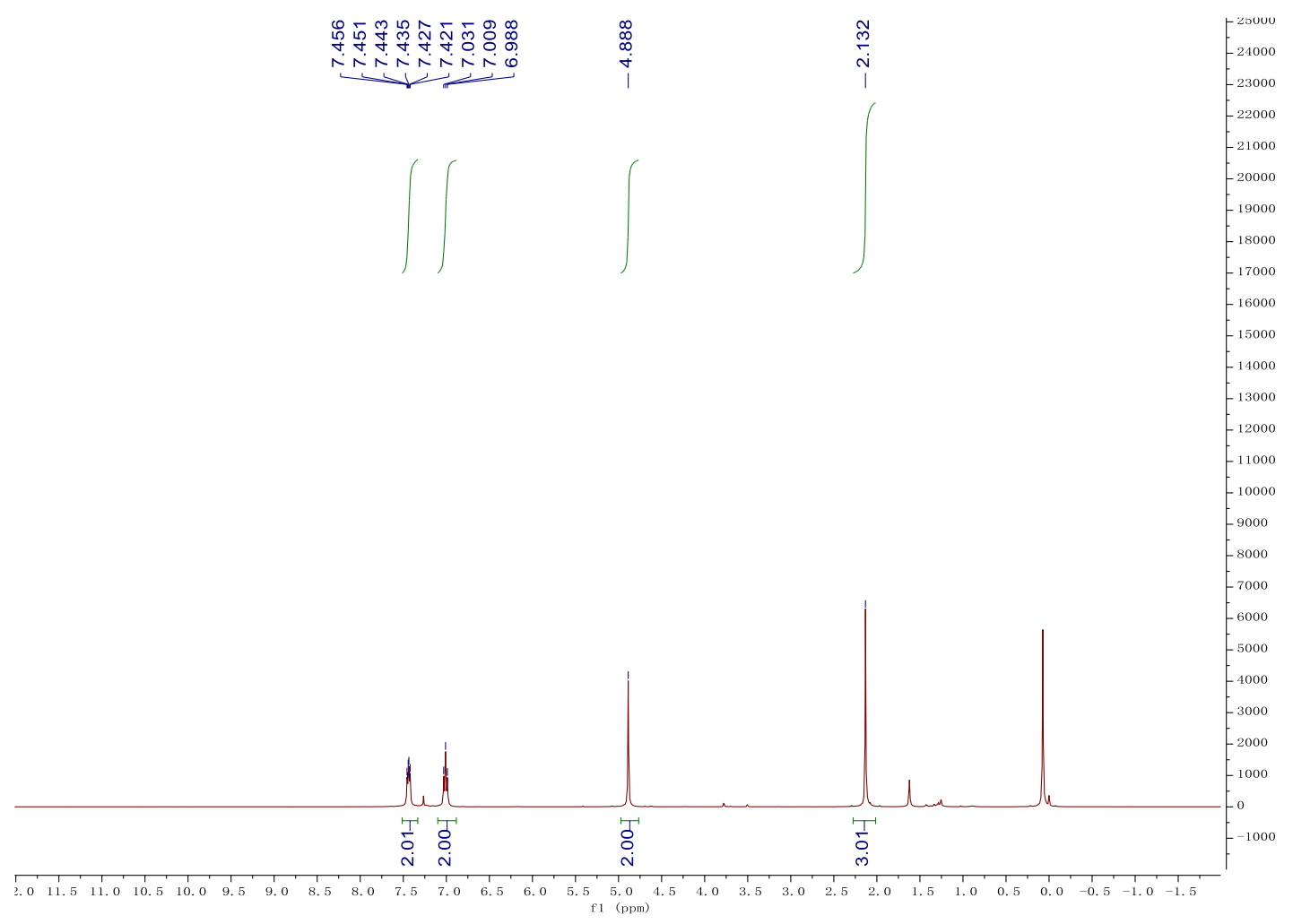




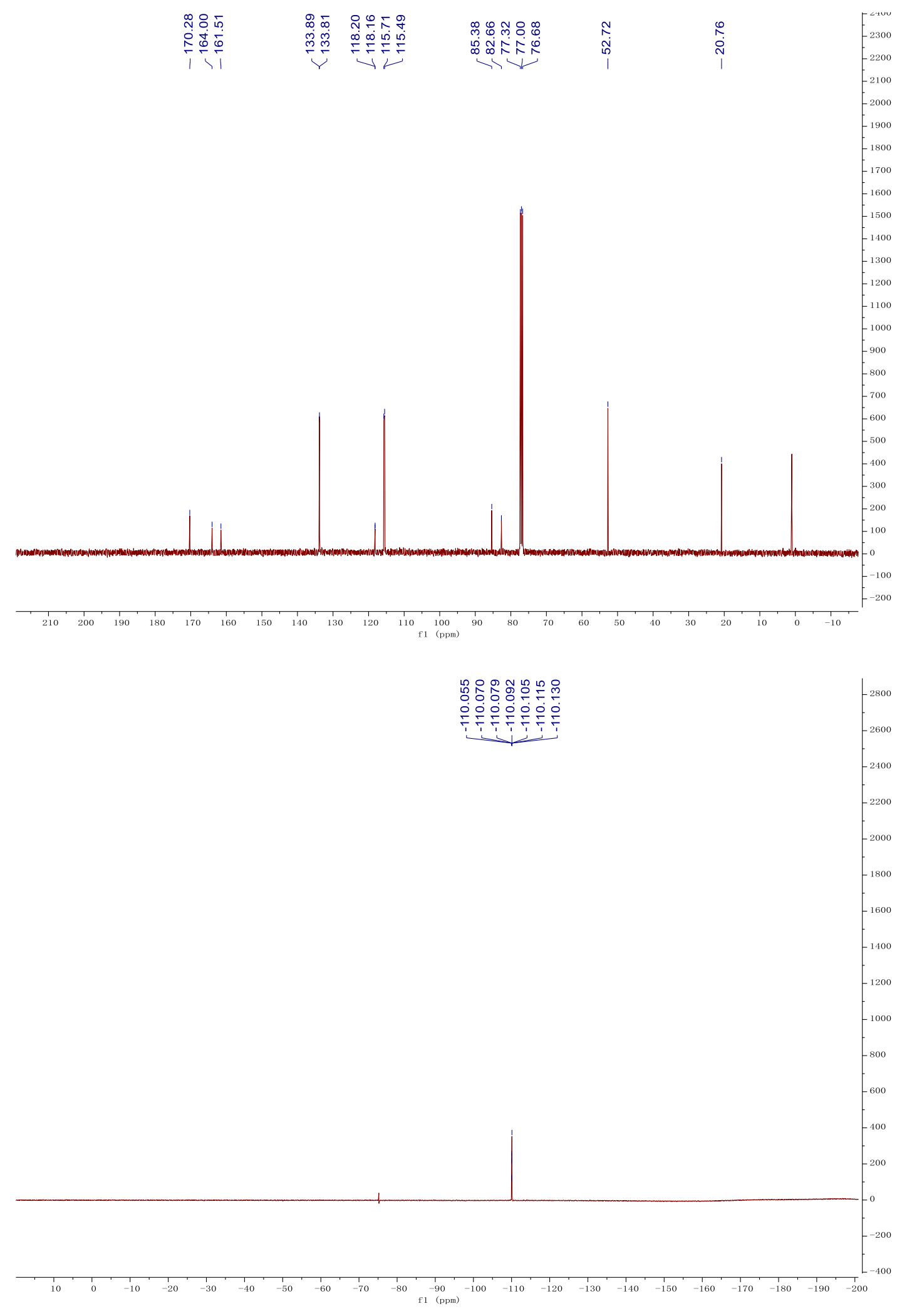




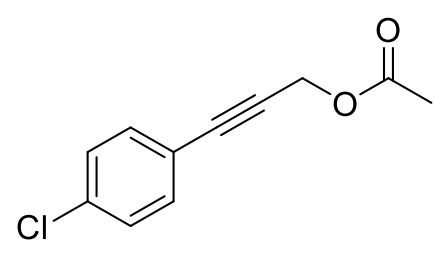

3-(4-Chlorophenyl)prop-2-yn-1-yl acetate (3h). A known compound $^{[7]}$. Yield: $38 \mathrm{mg}, 62 \%$. Colorless oil. ${ }^{1} \mathbf{H}$ NMR $\left(400 \mathrm{MHz}, \mathrm{CDCl}_{3}\right) \delta 7.38(\mathrm{~d}, J=8.0 \mathrm{~Hz}, 2 \mathrm{H}), 7.29(\mathrm{~d}, J=$ $8.4 \mathrm{~Hz}, 2 \mathrm{H}), 4.89$ (s, 2H), 2.14 (s, 3H); ${ }^{13} \mathrm{C}$ NMR (101 MHz, $\left.\mathrm{CDCl}_{3}\right) \delta 170.3,134.9,133.1,128.7,120.6,85.3,83.9,52.7,20.8$.

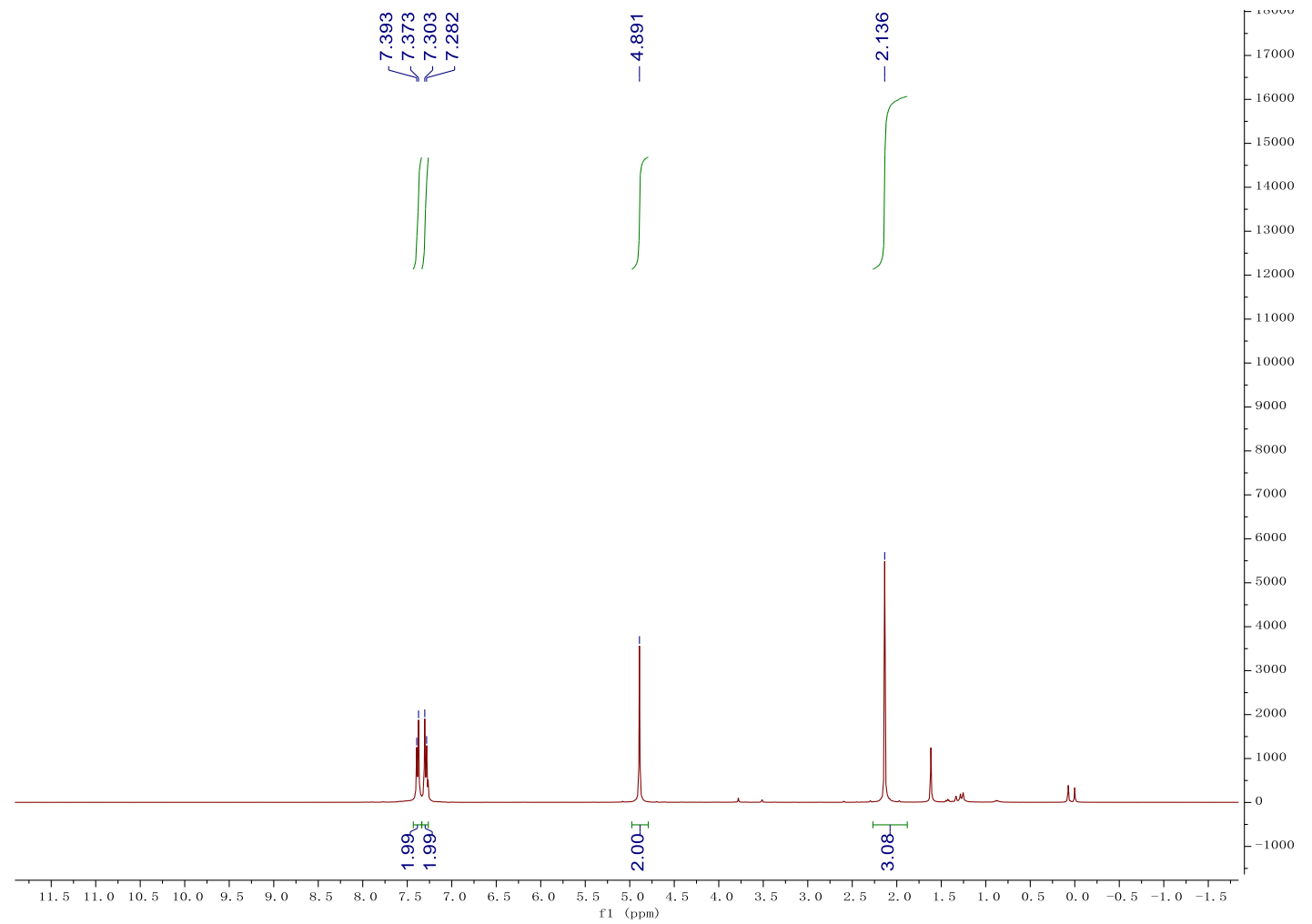




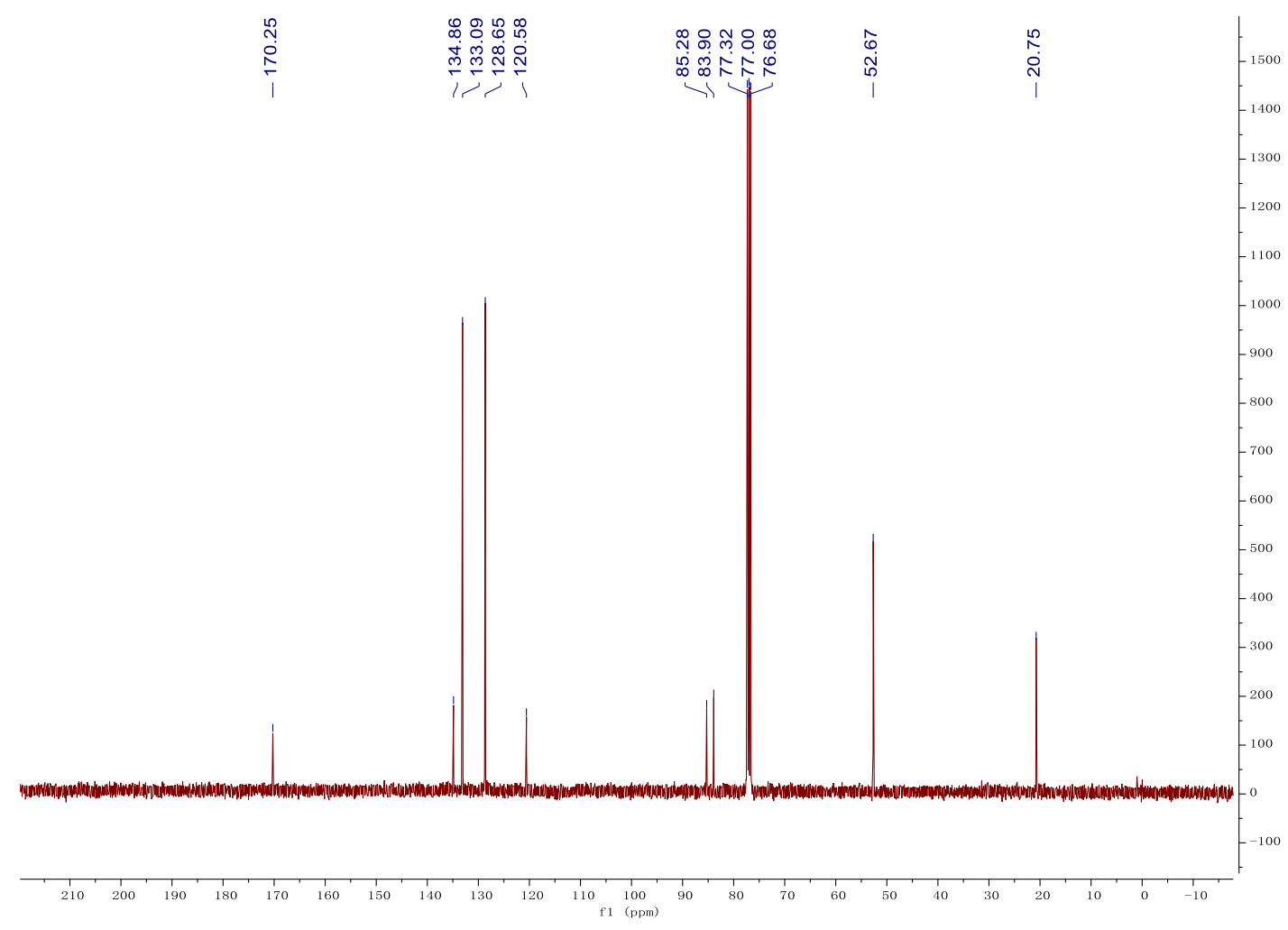

3-(4-Bromophenyl)prop-2-yn-1-yl acetate (3i). Yield: 44
$\mathrm{mg}, 60 \%$. White soild. M.p. $46-47{ }^{\circ} \mathrm{C} .{ }^{1} \mathbf{H}$ NMR $(400 \mathrm{MHz}$,
$\left.\mathrm{CDCl}_{3}\right) \delta 7.45(\mathrm{~d}, J=8.0 \mathrm{~Hz}, 2 \mathrm{H}), 7.31(\mathrm{~d}, J=8.4 \mathrm{~Hz}, 2 \mathrm{H})$,
$4.88(\mathrm{~s}, 2 \mathrm{H}), 2.14(\mathrm{~s}, 3 \mathrm{H}) ;{ }^{13} \mathrm{C} \mathbf{~ N M R}\left(101 \mathrm{MHz}, \mathrm{CDCl}_{3}\right) \delta$ 170.2, 133.3, 131.6, 123.1, 121.1, 85.3, 84.1, 52.7, 20.8. IR (KBr): v 3071, 2928, 2854 , 1737, 1484, 1252, 1026, 907, 827, $809 \mathrm{~cm}^{-1}$. HRMS (ESI) calcd for $\left[\mathrm{C}_{11} \mathrm{H}_{9} \mathrm{BrO}_{2}+\mathrm{K}\right]^{+}$ requires 290.9418 , found $290.9418\left[\mathrm{M}^{+}+\mathrm{K}\right]$. 

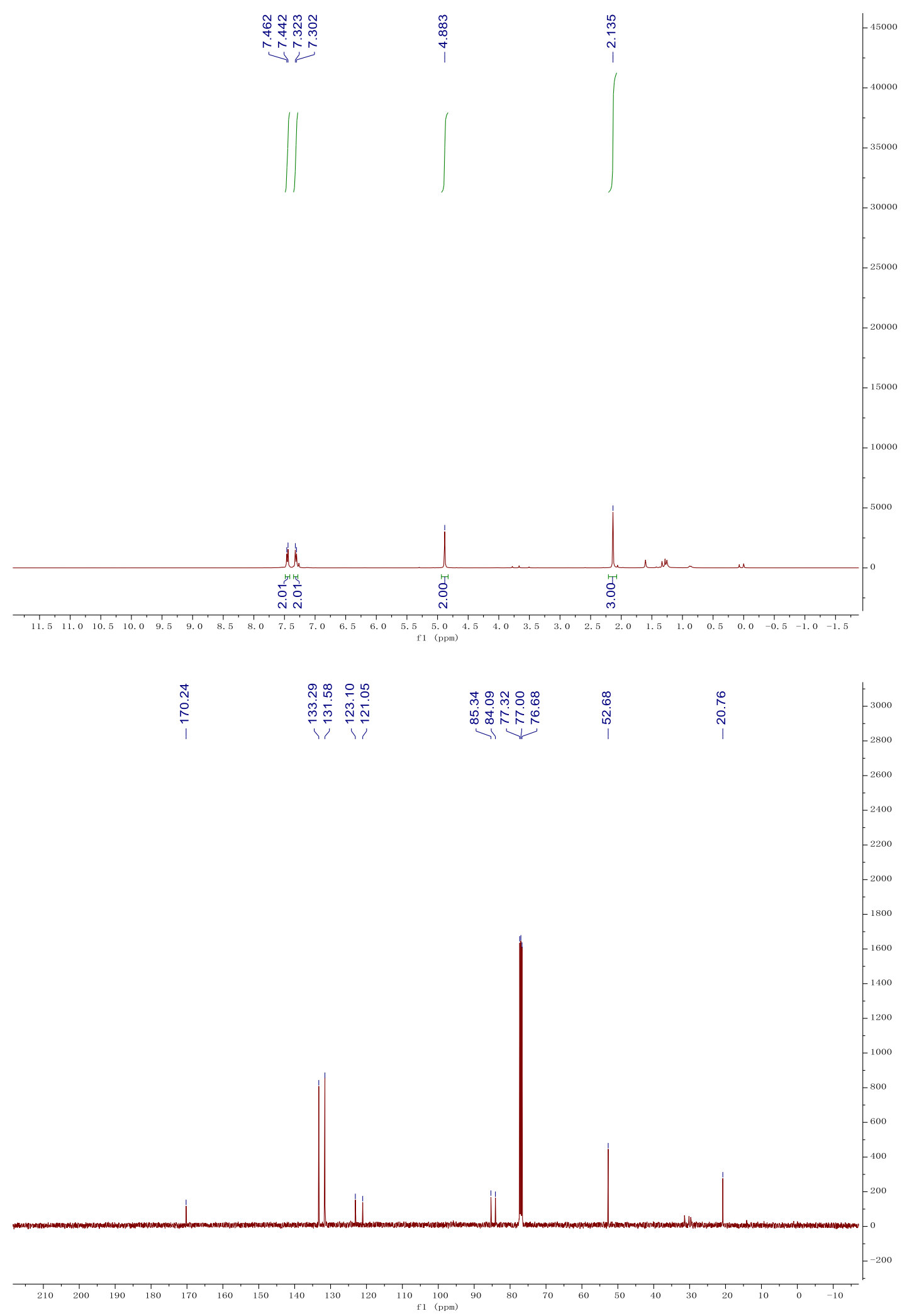


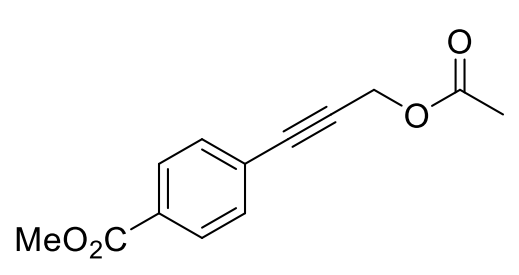

Methyl 4-(3-acetoxyprop-1-yn-1-yl)benzoate (3j).

Yield: $40 \mathrm{mg}, 58 \%$. White soild. M.p. $38-39{ }^{\circ} \mathrm{C} .{ }^{1} \mathbf{H}$ NMR (400 MHz, CDCl3) $\delta 7.99(\mathrm{~d}, J=8.4 \mathrm{~Hz}, 2 \mathrm{H})$, $7.51(\mathrm{~d}, J=7.6 \mathrm{~Hz}, 2 \mathrm{H}), 4.92(\mathrm{~s}, 2 \mathrm{H}), 3.92(\mathrm{~s}, 3 \mathrm{H}), 2.14$ $(\mathrm{s}, 3 \mathrm{H}) ;{ }^{13} \mathrm{C}$ NMR $\left(101 \mathrm{MHz}, \mathrm{CDCl}_{3}\right) \delta 170.2,166.4,131.8,130.0,129.4,126.7,85.8$, 85.6, 52.6, 52.2, 20.7. IR (KBr): v 2927, 2853, 1732, 1438, 1282, 1106, 852, $764 \mathrm{~cm}^{-1}$. HRMS (ESI) calcd for $\left[\mathrm{C}_{13} \mathrm{H}_{20} \mathrm{O}_{4}+\mathrm{Na}\right]^{+}$requires 255.0628 , found $255.0628\left[\mathrm{M}^{+}+\mathrm{Na}\right]$.

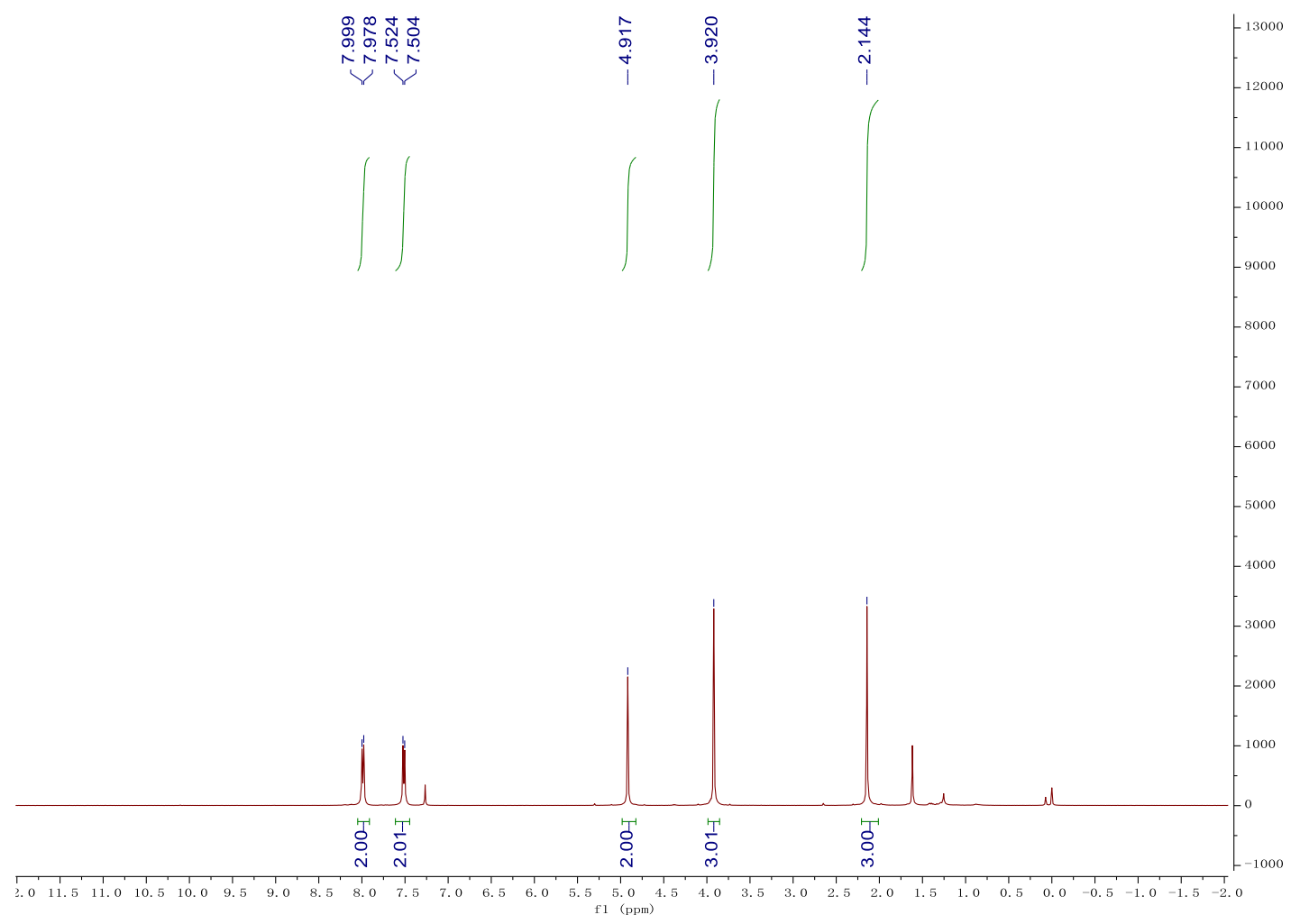




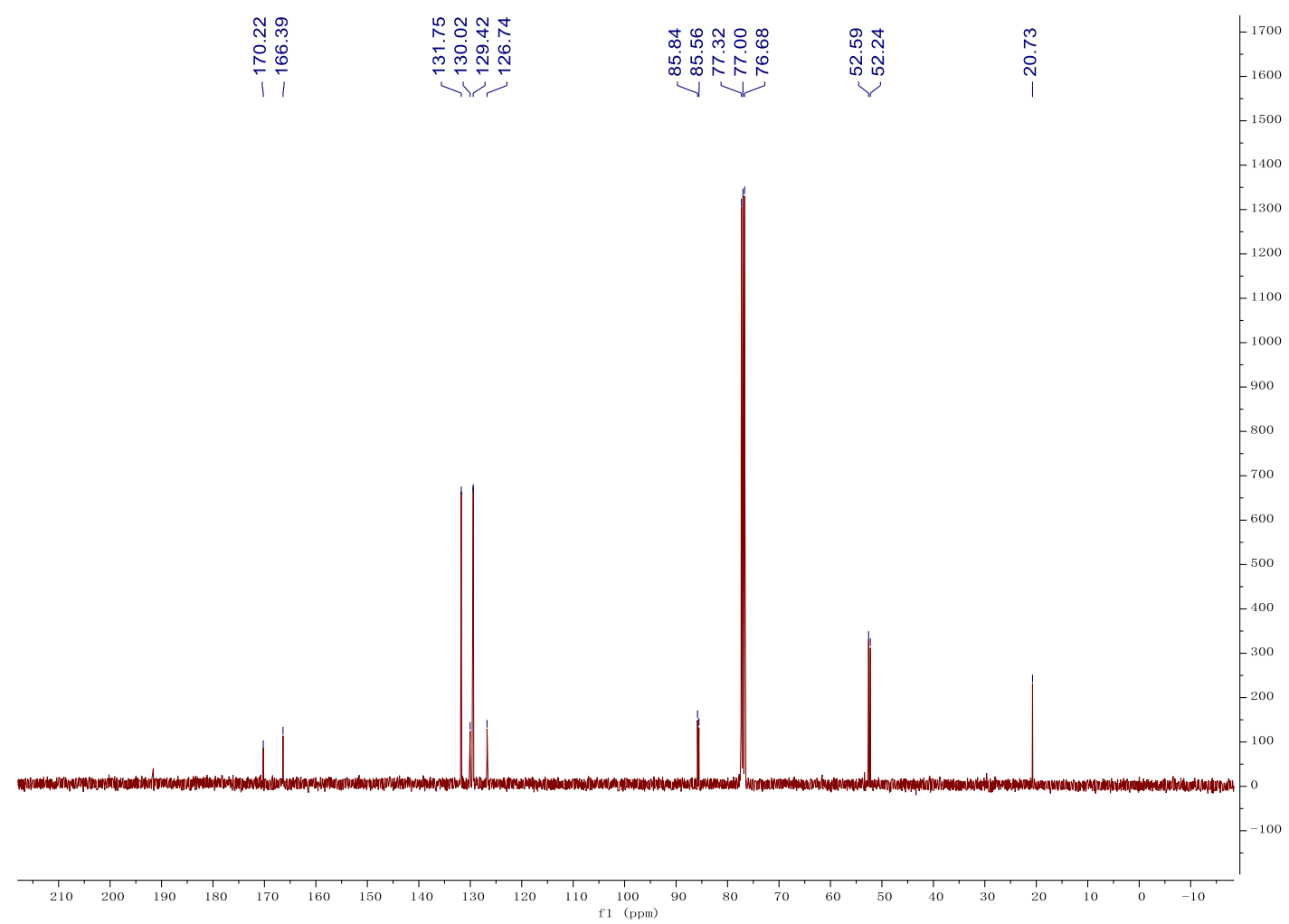

3-(m-Tolyl)prop-2-yn-1-yl acetate (3k). Yield: $40 \mathrm{mg}, 72 \%$.
Colorless oil. ${ }^{\mathbf{1}} \mathbf{H} \mathbf{N M R}\left(400 \mathrm{MHz}, \mathrm{CDCl}_{3}\right) \delta 7.29-7.25(\mathrm{~m}, 2 \mathrm{H})$,
$7.19(\mathrm{dd}, J=7.6,7.6 \mathrm{~Hz}, 1 \mathrm{H}), 7.14(\mathrm{~d}, J=7.6 \mathrm{~Hz}, 1 \mathrm{H}), 4.90(\mathrm{~s}$,
$2 \mathrm{H}), 2.32(\mathrm{~s}, 3 \mathrm{H}), 2.12(\mathrm{~s}, 3 \mathrm{H}) ;{ }^{13} \mathbf{C} \mathbf{N M R}\left(101 \mathrm{MHz}, \mathrm{CDCl}_{3}\right) \delta$ 170.3, 138.0, 132.4, 129.6, 128.9, 128.2, 121.9, 86.6, 82.5, 52.9, 21.1, 20.8. IR (KBr): v 3037, 2927, 2859, 1747, 1360, 1224, 1035, 787, $692 \mathrm{~cm}^{-1}$. HRMS (ESI) calcd for $\left[\mathrm{C}_{12} \mathrm{H}_{12} \mathrm{O}_{2}+\mathrm{Na}\right]^{+}$requires 211.0730, found 211.0730 [ $\left.\mathrm{M}^{+}+\mathrm{Na}\right]$. 

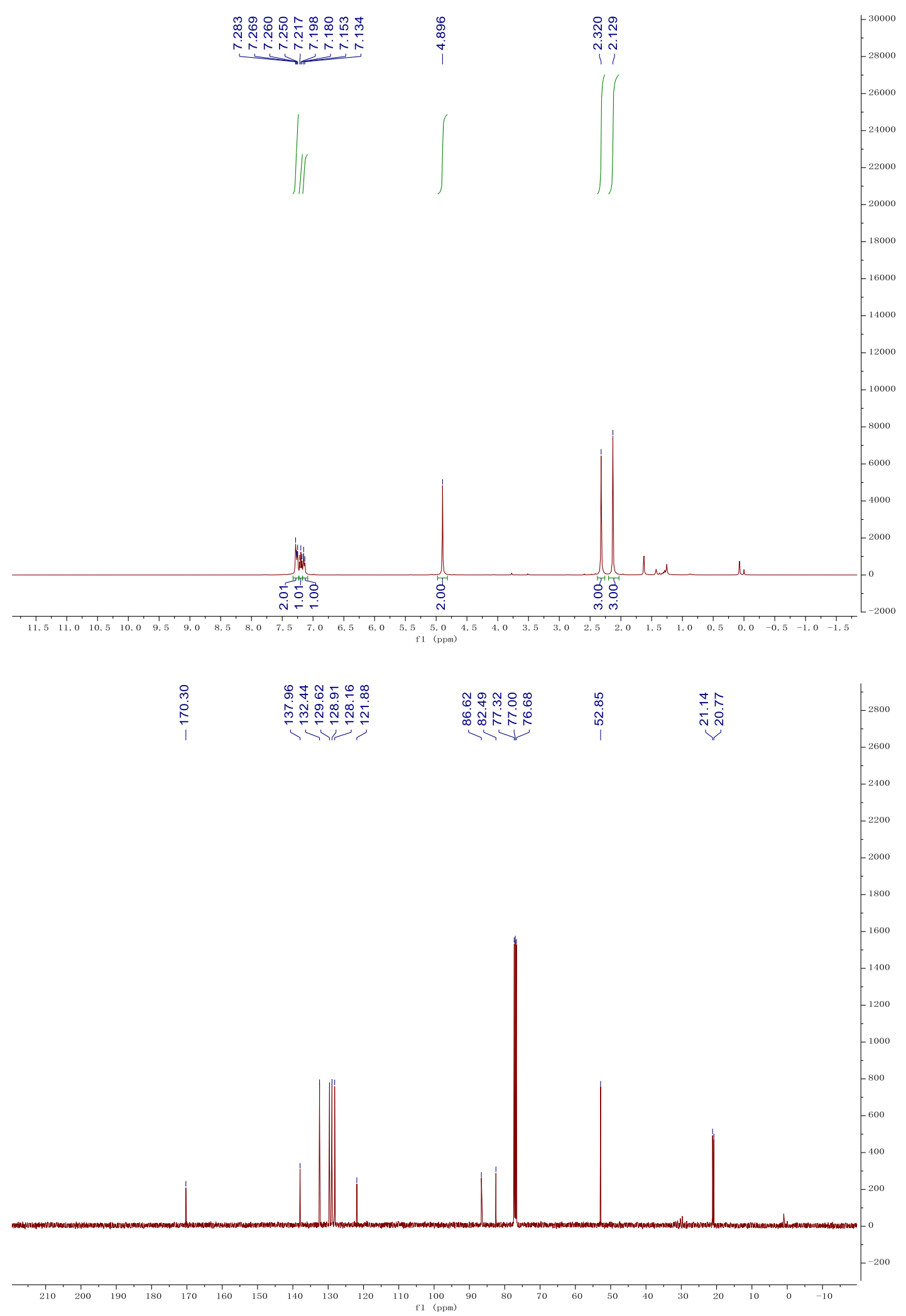


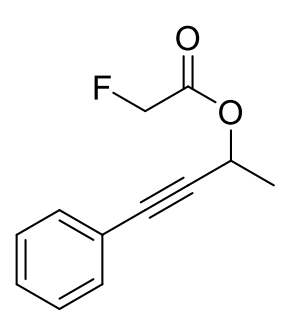

4-Phenylbut-3-yn-2-yl 2-fluoroacetate (3l). Yield: $40 \mathrm{mg}, 65 \%$.

Colorless oil. ${ }^{1} \mathbf{H}$ NMR $\left(400 \mathrm{MHz}, \mathrm{CDCl}_{3}\right) \delta$ 7.46-7.42 (m, 2H),

7.34-7.28 (m, 3H), $5.82(\mathrm{q}, J=6.8 \mathrm{~Hz}, 1 \mathrm{H}), 4.89$ (dd, $J=46.8,2.0$ $\mathrm{Hz}, 1 \mathrm{H}), 1.64(\mathrm{~d}, J=6.8 \mathrm{~Hz}, 3 \mathrm{H}) ;{ }^{13} \mathbf{C} \mathbf{N M R}\left(101 \mathrm{MHz}, \mathrm{CDCl}_{3}\right) \delta$ $166.8\left(J_{\mathrm{CF}}=22.2 \mathrm{~Hz}\right), 131.9,128.8,128.7,121.9,86.2,85.5,77.5$ $\left(J_{\mathrm{CF}}=182.8 \mathrm{~Hz}\right), 62.2,21.4 ;{ }^{19} \mathbf{F} \mathbf{N M R}\left(376 \mathrm{MHz}, \mathrm{CDCl}_{3}\right) \delta-179.2(\mathrm{~d}, J=48.5 \mathrm{~Hz}$, 1F). IR (KBr): v 3061, 2992, 2942, 1770, 1443, 1211, 1083, 948, $759 \mathrm{~cm}^{-1} . \mathbf{H R M S}$ (ESI) calcd for $\left[\mathrm{C}_{12} \mathrm{H}_{21} \mathrm{FO}_{2}+\mathrm{Na}\right]^{+}$requires 229.0635, found $229.0635\left[\mathrm{M}^{+}+\mathrm{Na}\right]$.

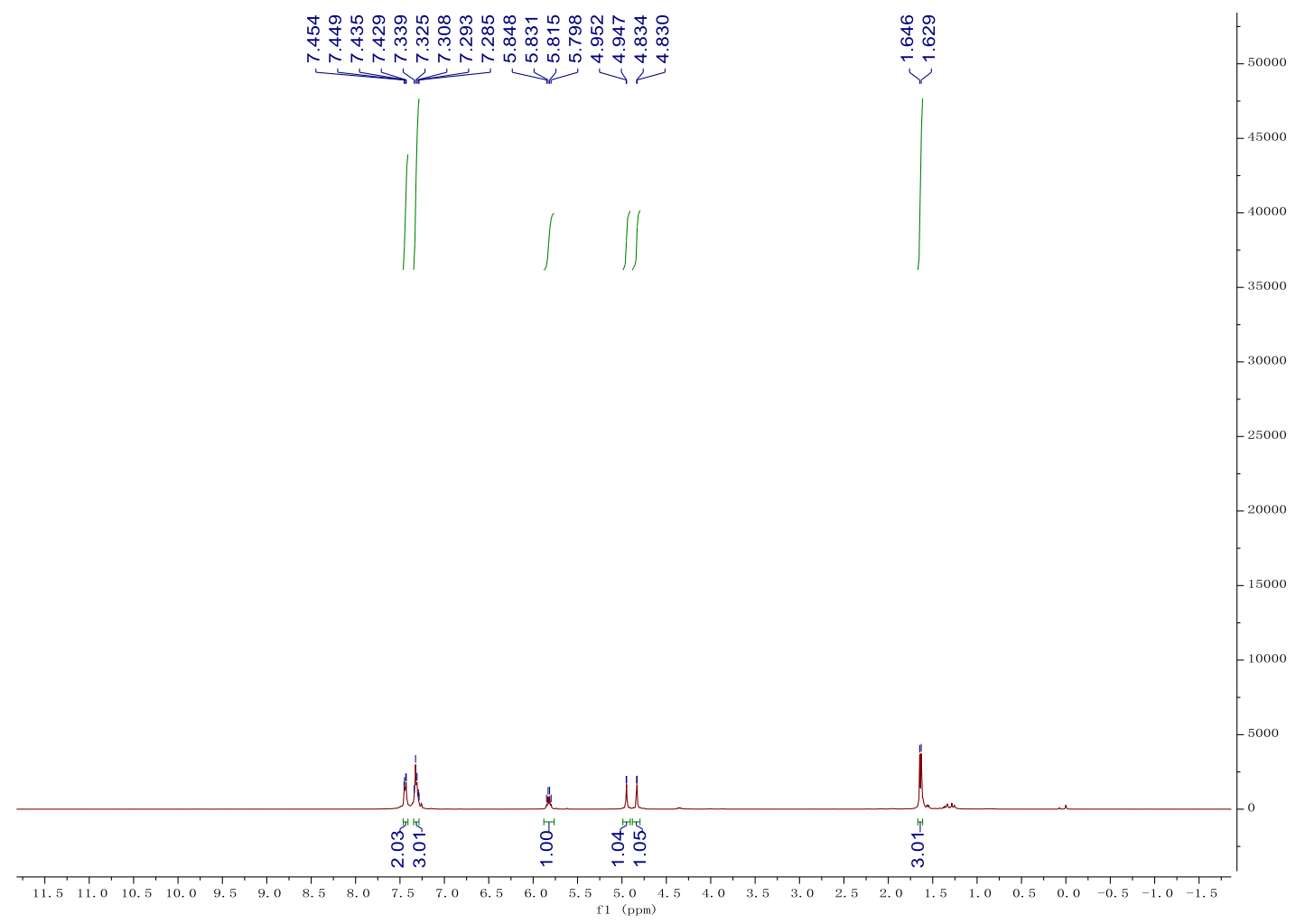




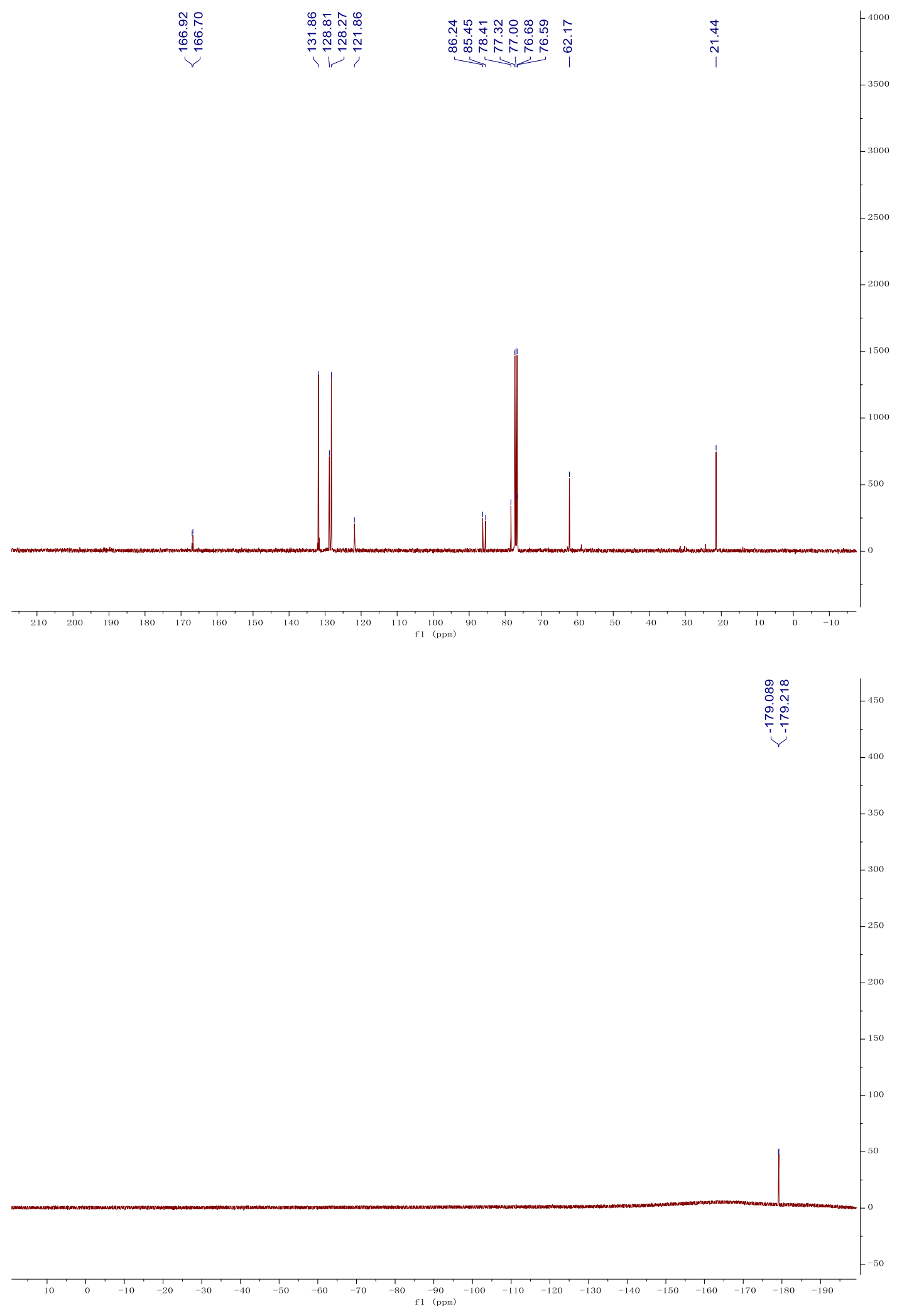




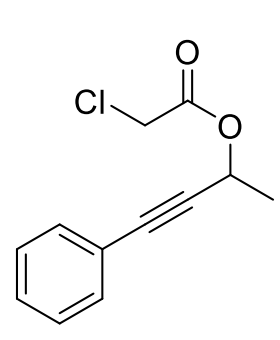

4-Phenylbut-3-yn-2-yl 2-chloroacetate (3m). A known compound ${ }^{[8]}$. Yield: $45 \mathrm{mg}, 65 \%$. Colorless oil. ${ }^{1} \mathbf{H}$ NMR $\left(400 \mathrm{MHz}, \mathrm{CDCl}_{3}\right) \delta$ 7.46-7.43 (m, 2H), 7.35-7.30 (m, 3H), 5.76 (q, $J=6.8 \mathrm{~Hz}, 1 \mathrm{H}), 4.11$ $(\mathrm{s}, 2 \mathrm{H}), 1.63(\mathrm{~d}, J=6.8 \mathrm{~Hz}, 3 \mathrm{H}) ;{ }^{13} \mathbf{C} \mathbf{N M R}\left(101 \mathrm{MHz}, \mathrm{CDCl}_{3}\right) \delta$ 166.3. 131.9, 128.8, 128.3, 121.9, 86.3, 85.5, 62.9, 40.9, 21.4.

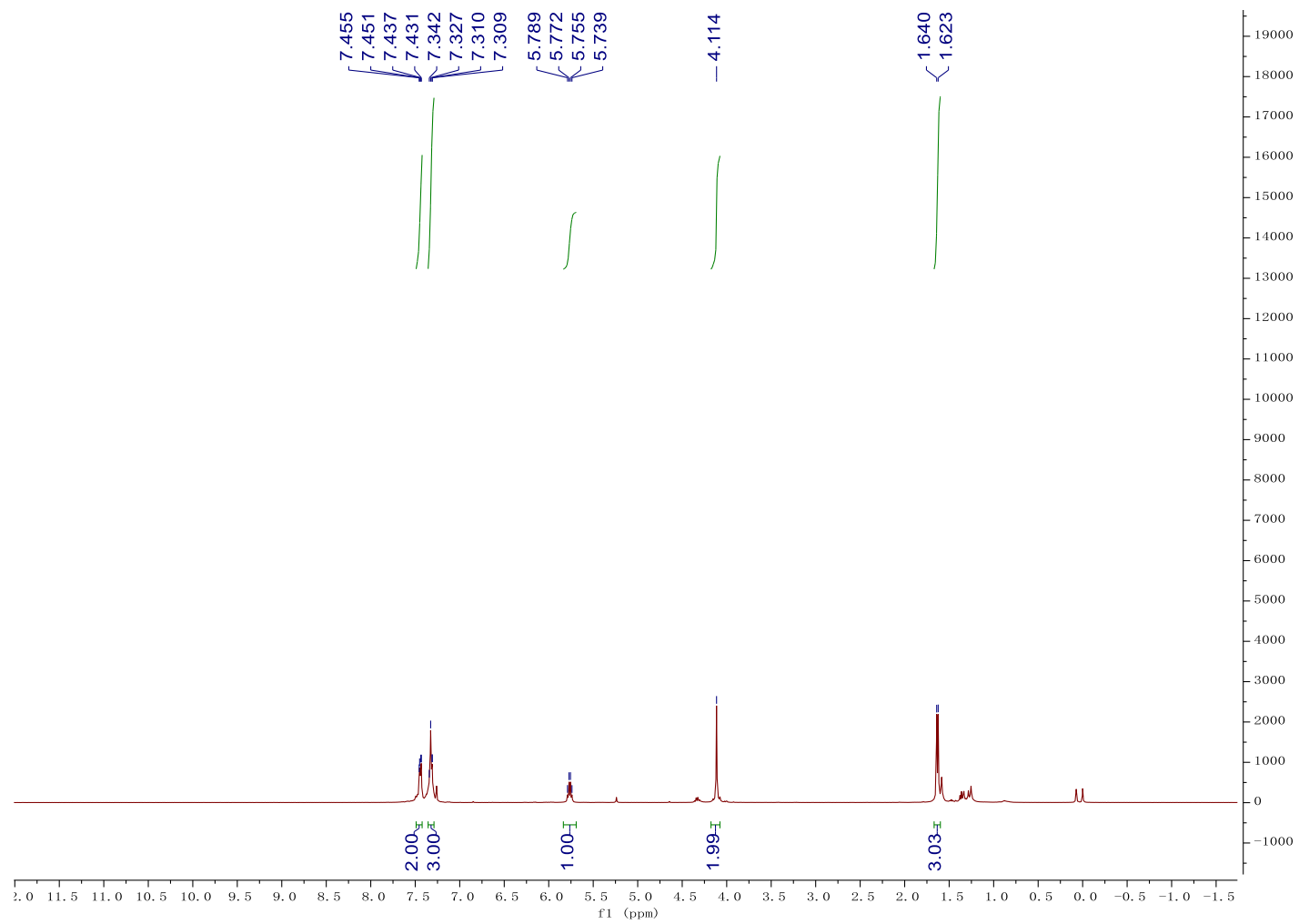



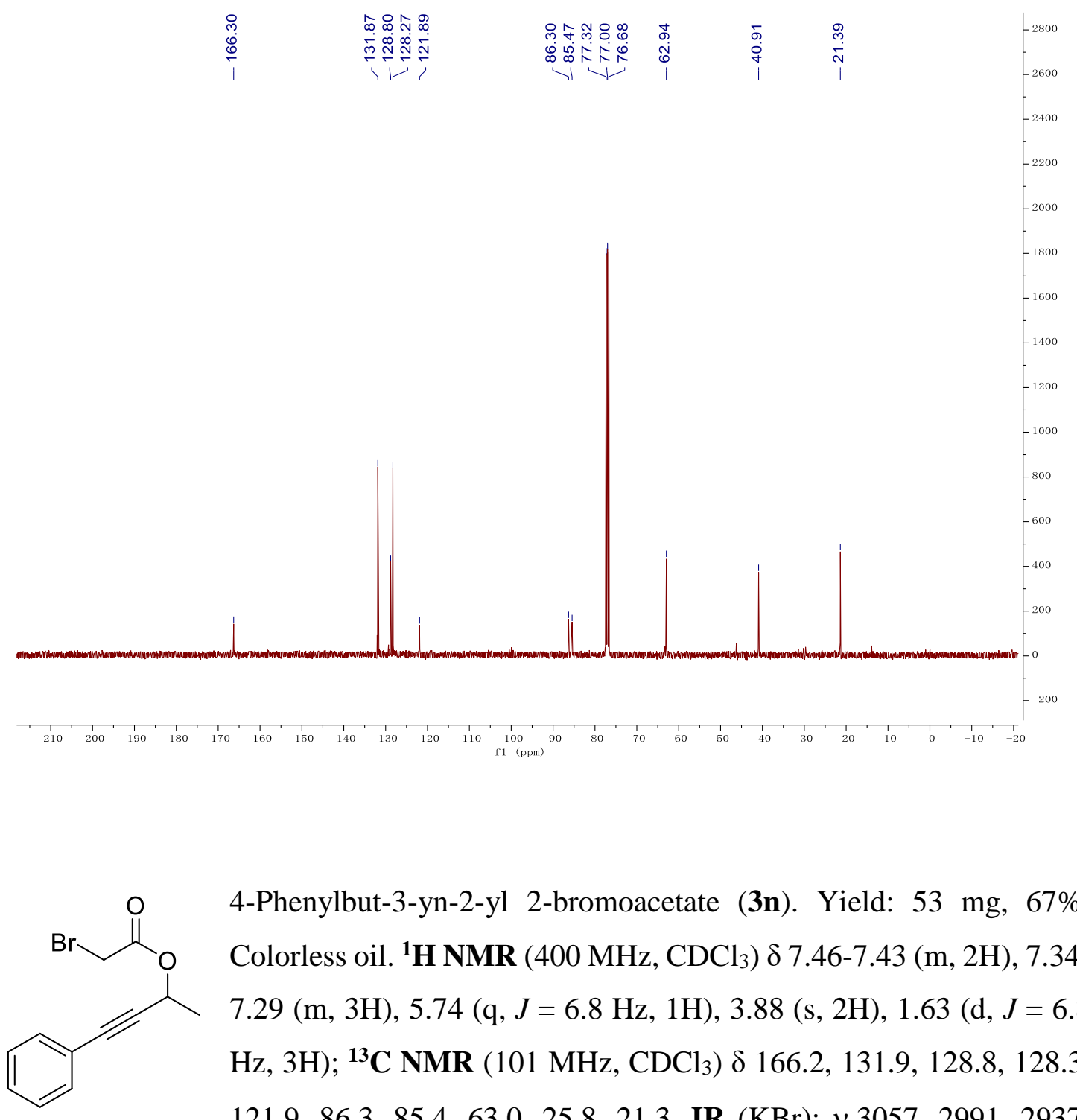

4-Phenylbut-3-yn-2-yl 2-bromoacetate (3n). Yield: $53 \mathrm{mg}, 67 \%$. Colorless oil. ${ }^{1} \mathbf{H}$ NMR (400 MHz, $\left.\mathrm{CDCl}_{3}\right) \delta$ 7.46-7.43 (m, 2H), 7.34$7.29(\mathrm{~m}, 3 \mathrm{H}), 5.74(\mathrm{q}, J=6.8 \mathrm{~Hz}, 1 \mathrm{H}), 3.88(\mathrm{~s}, 2 \mathrm{H}), 1.63(\mathrm{~d}, J=6.8$ $\mathrm{Hz}, 3 \mathrm{H}) ;{ }^{13} \mathrm{C}$ NMR $\left(101 \mathrm{MHz}, \mathrm{CDCl}_{3}\right) \delta 166.2,131.9,128.8,128.3$, 121.9, 86.3, 85.4, 63.0, 25.8, 21.3. IR (KBr): v 3057, 2991, 2937, 1743, 1445, 1273, 1086, 956, $758 \mathrm{~cm}^{-1}$. HRMS (ESI) calcd for $\left[\mathrm{C}_{12} \mathrm{H}_{11} \mathrm{BrO}_{2}+\mathrm{K}\right]^{+}$ requires 304.9574 , found $304.9575\left[\mathrm{M}^{+}+\mathrm{K}\right]$ 

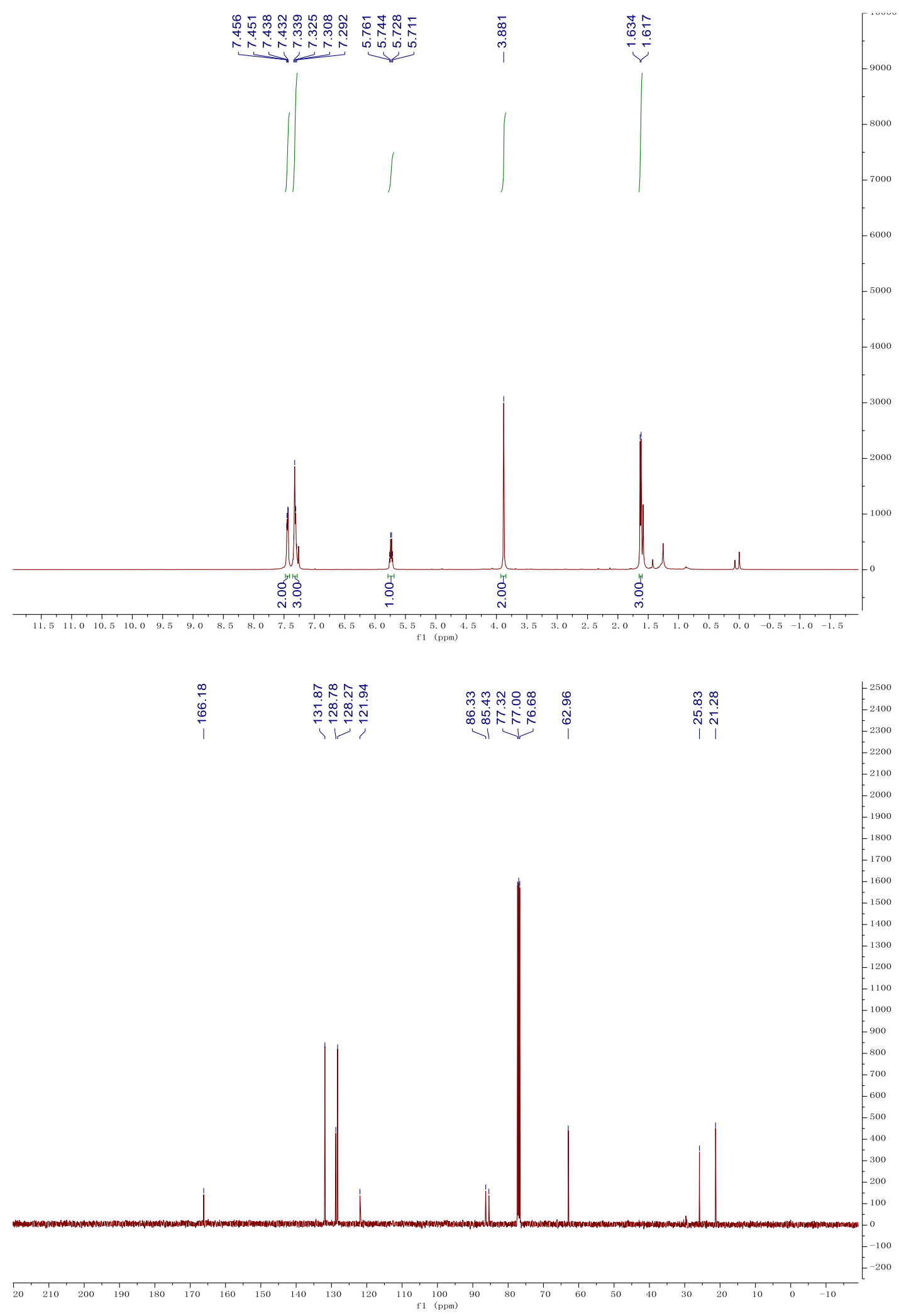
<smiles>CC(=O)OC(C)/C=C/c1ccccc1</smiles>

(E)-4-Phenylbut-3-en-2-yl acetate (3o). A known compound $^{[9]}$. Yield: $28 \mathrm{mg}, 49 \%$. Colorless oil. ${ }^{\mathbf{1}} \mathbf{H}$ NMR $\left(400 \mathrm{MHz}, \mathrm{CDCl}_{3}\right) \delta 7.38(\mathrm{~d}, J=7.6 \mathrm{~Hz}, 2 \mathrm{H}), 7.31(\mathrm{dd}, J=$ 7.2, 7.2 Hz, 2H), $7.25(\mathrm{~d}, J=6.4 \mathrm{~Hz}, 1 \mathrm{H}), 6.59$ (d, $J=16.0 \mathrm{~Hz}, 1 \mathrm{H}), 6.21$ (dd, $J=$ 16.0, $6.8 \mathrm{~Hz}, 1 \mathrm{H}), 5.53(\mathrm{~m}, 1 \mathrm{H}), 2.07(\mathrm{~s}, 3 \mathrm{H}), 1.41(\mathrm{~d}, J=6.4 \mathrm{~Hz}, 3 \mathrm{H}) ;{ }^{13} \mathbf{C}$ NMR $(101$ $\left.\mathrm{MHz}, \mathrm{CDCl}_{3}\right) \delta 170.3,136.3,131.5,128.7,128.5,127.9,126.5,70.1,21.4,20.3$.

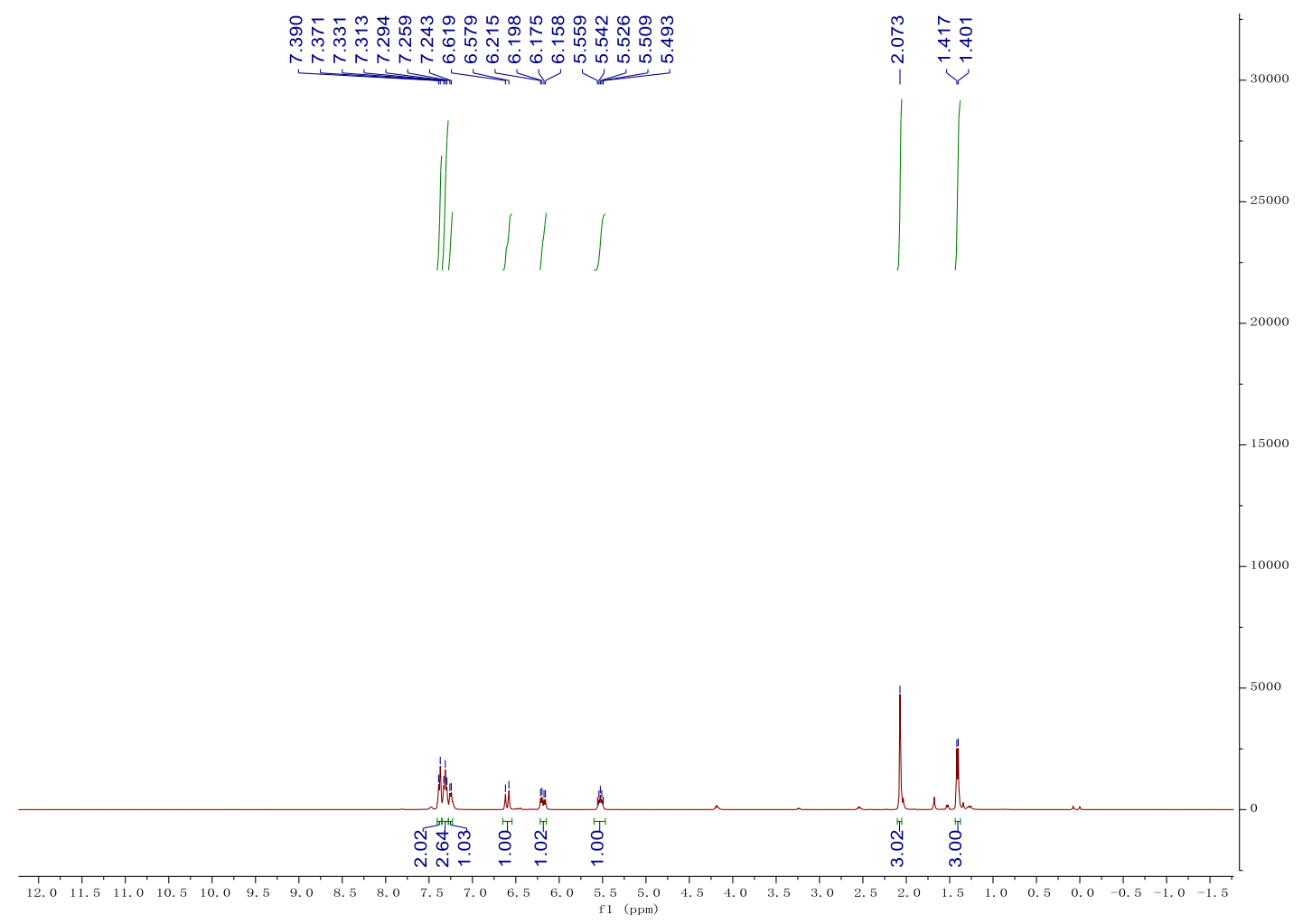




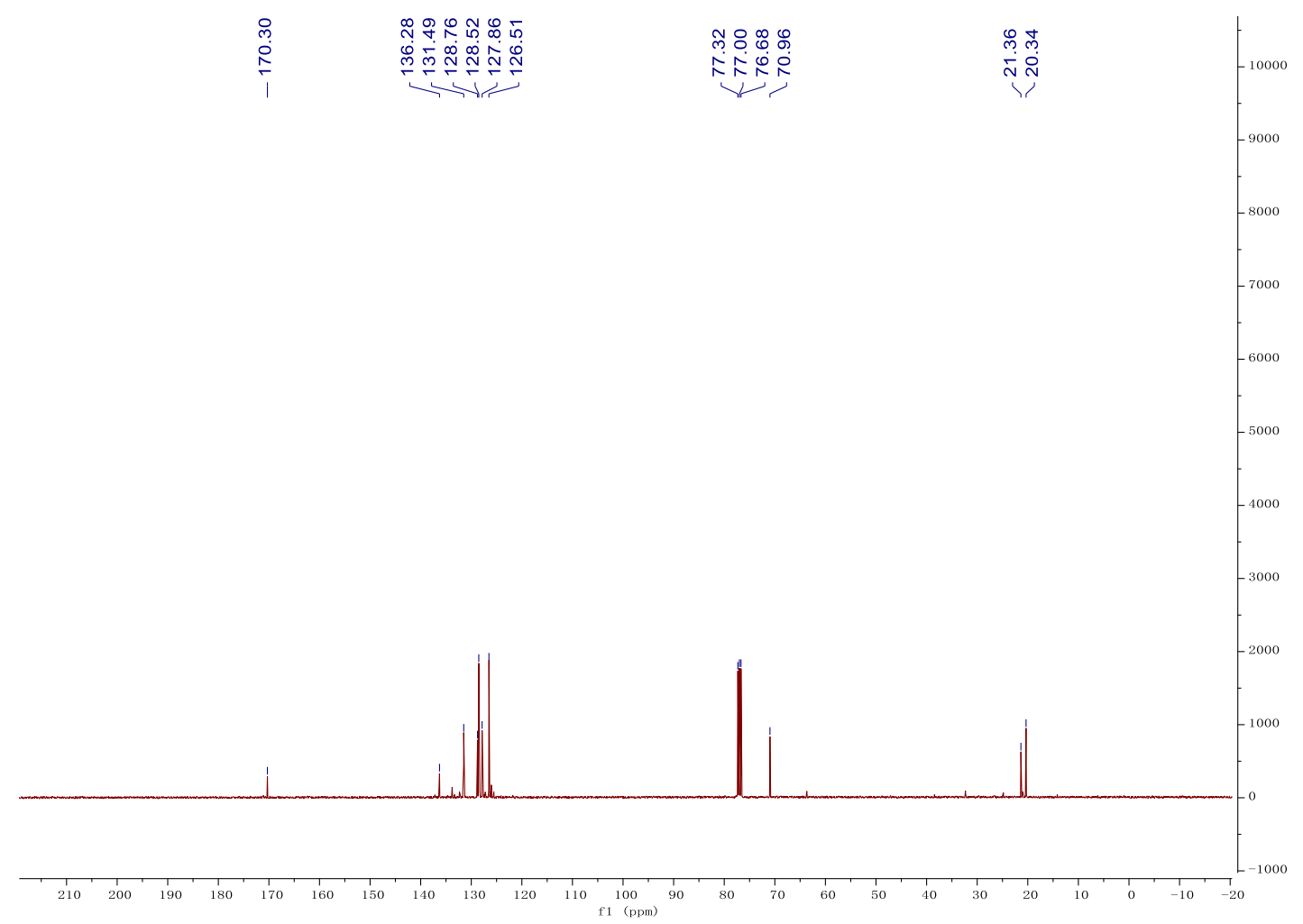

known compound ${ }^{[10]}$ Yield: $35 \mathrm{mg}, 53 \%$. Colorless oil. ${ }^{\mathbf{1}} \mathbf{H}$
$\mathbf{N}\left(400 \mathrm{MHz}, \mathrm{CDCl}_{3}\right) \delta 7.32-7.26(\mathrm{~m}, 4 \mathrm{H}) .6 .55(\mathrm{~d}, J$ $=16.0 \mathrm{~Hz}, 1 \mathrm{H}), 6.16(\mathrm{dd}, J=16.0,6.4 \mathrm{~Hz}, 1 \mathrm{H}), 5.55-5.47(\mathrm{~m}, 1 \mathrm{H}), 2.08(\mathrm{~s}, 3 \mathrm{H}), 1.40$ $($ d. $J=6.8 \mathrm{~Hz}, 3 \mathrm{H}) ;{ }^{13} \mathrm{C}$ NMR $\left(101 \mathrm{MHz}, \mathrm{CDCl}_{3}\right) \delta 170.3,134.8,133.5,130.2,129.5$, 128.7, 127.7, 70.8, 21.3, 20.3. 

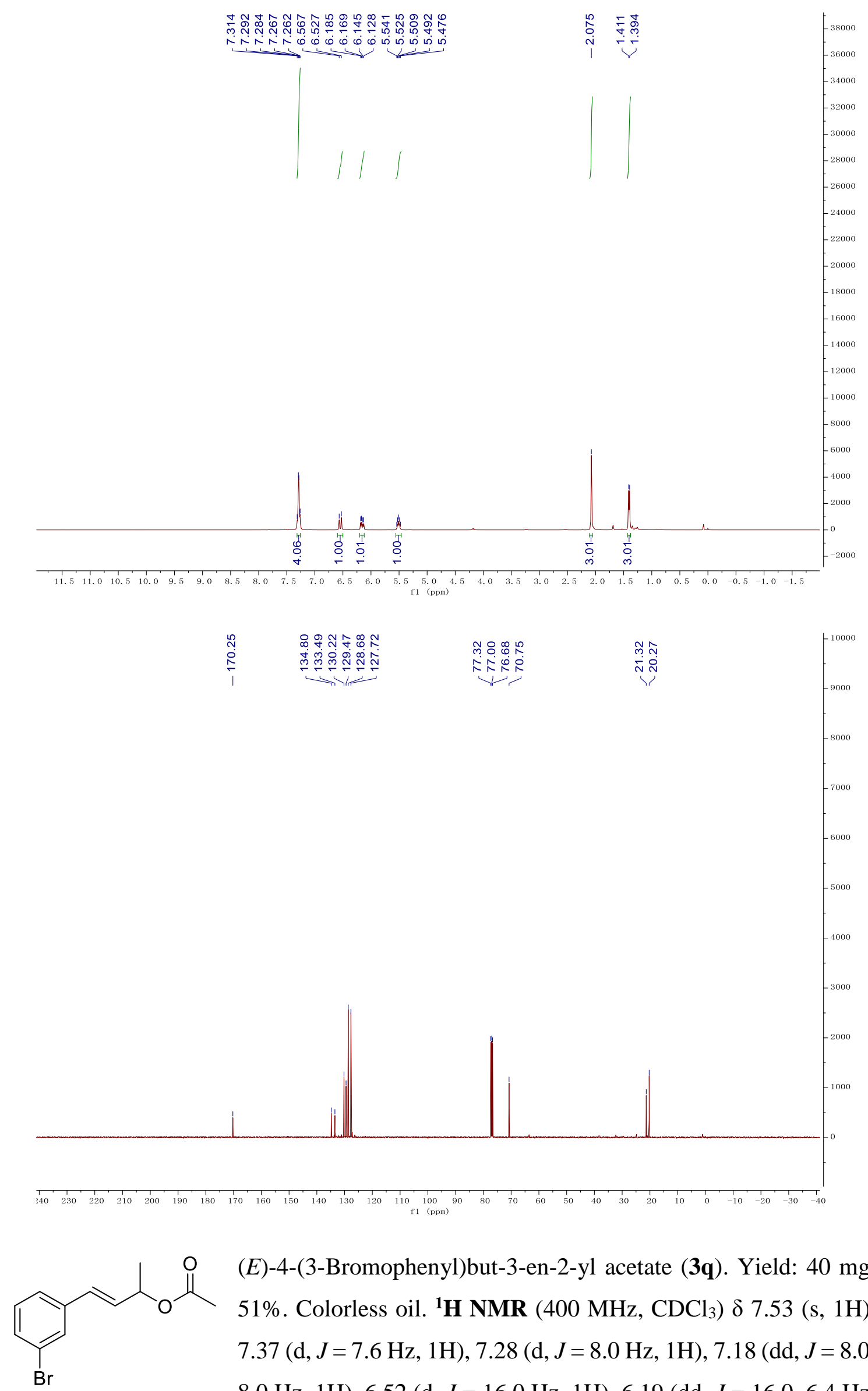

(E)-4-(3-Bromophenyl)but-3-en-2-yl acetate (3q). Yield: $40 \mathrm{mg}$, $51 \%$. Colorless oil. ${ }^{1} \mathbf{H}$ NMR (400 MHz, $\left.\mathrm{CDCl}_{3}\right) \delta 7.53(\mathrm{~s}, 1 \mathrm{H})$, $7.37(\mathrm{~d}, J=7.6 \mathrm{~Hz}, 1 \mathrm{H}), 7.28(\mathrm{~d}, J=8.0 \mathrm{~Hz}, 1 \mathrm{H}), 7.18$ (dd, $J=8.0$, $8.0 \mathrm{~Hz}, 1 \mathrm{H}), 6.52$ (d, $J=16.0 \mathrm{~Hz}, 1 \mathrm{H}), 6.19$ (dd, $J=16.0,6.4 \mathrm{~Hz}$, 
1H), 5.55-5.47 (m, 1H), $2.08(\mathrm{~s}, 3 \mathrm{H}), 1.40(\mathrm{~d}, J=6.4 \mathrm{~Hz}, 3 \mathrm{H}) ;{ }^{13} \mathrm{C}$ NMR (101 MHz, $\left.\mathrm{CDCl}_{3}\right) \delta 170.2,138.5,130.7,130.4,130.0,129.9,129.3,125.3,122.7,70.6,21.3,20.3$. IR (KBr): v 3066, 2981, 2932, 1737, 1475, 1371, 1241, 1043, 959, $778 \mathrm{~cm}^{-1}$. HRMS (ESI) calcd for $\left[\mathrm{C}_{12} \mathrm{H}_{13} \mathrm{BrO}_{2}+\mathrm{Na}\right]^{+}$requires 290.9991, found $290.9993\left[\mathrm{M}^{+}+\mathrm{Na}\right]$.

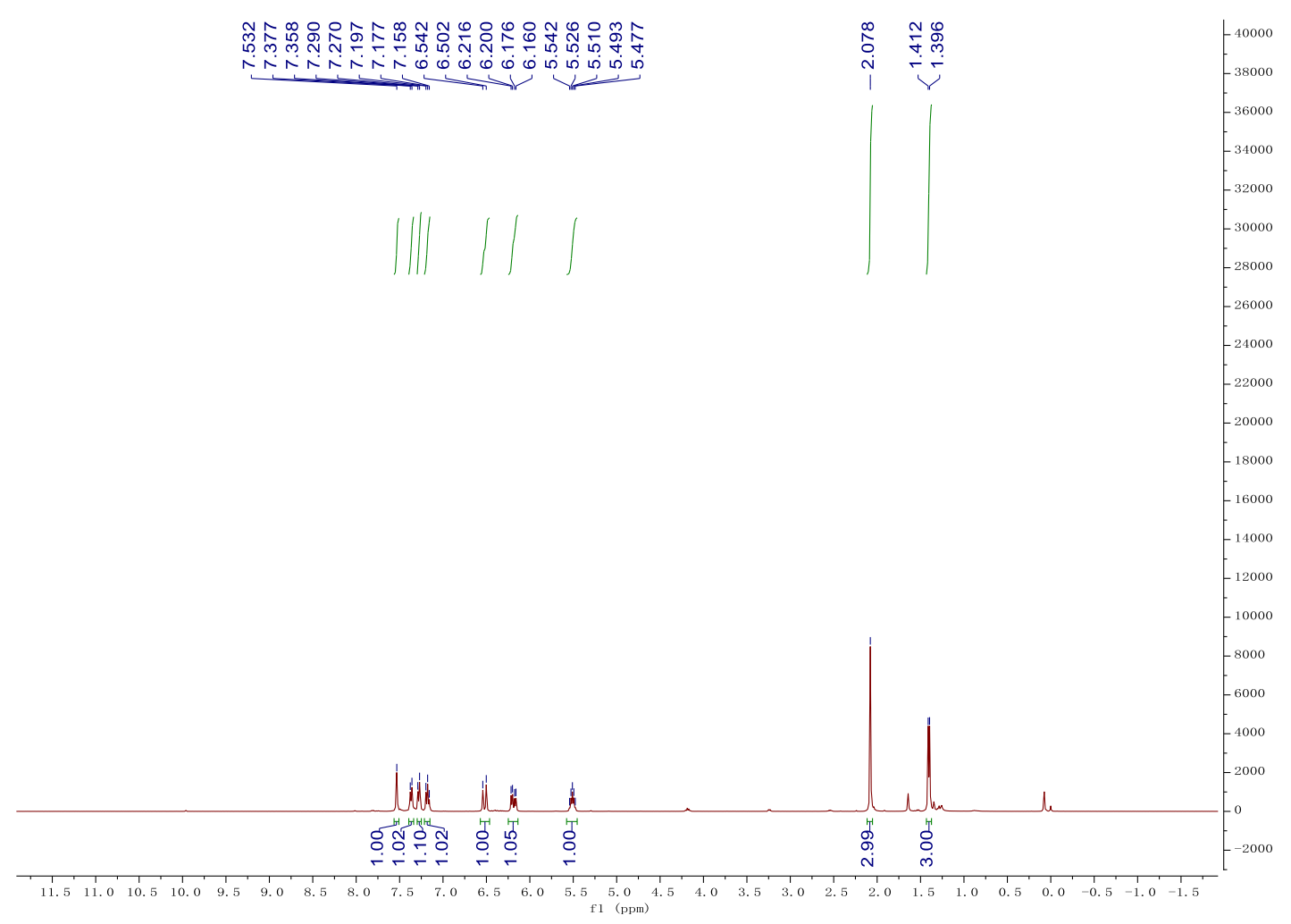




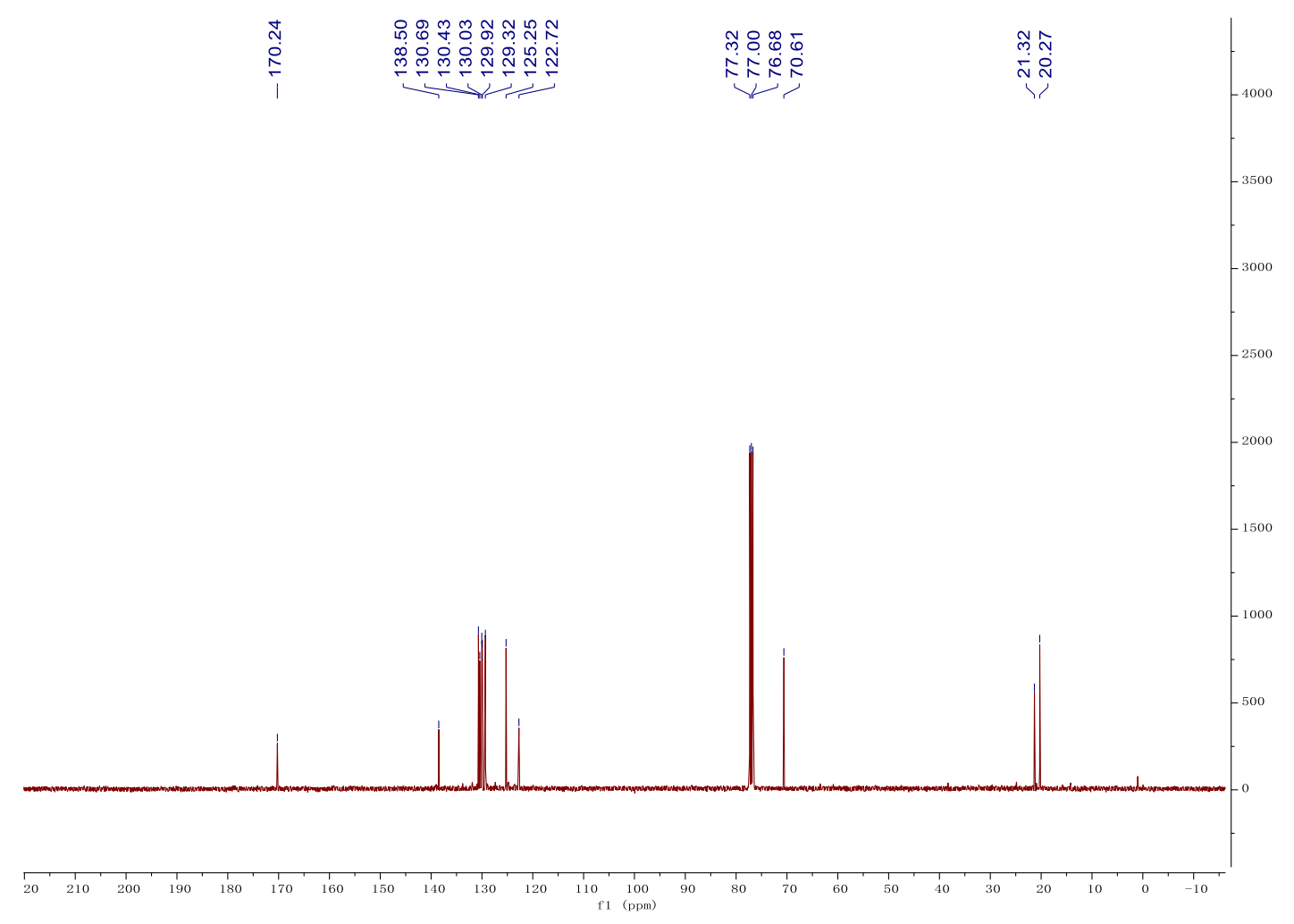

4-Phenylbut-3-yn-2-yl 2-azidoacetate (4). Yield: $21 \mathrm{mg}$,
$92 \%$. Colorless oil. ${ }^{\mathbf{1}} \mathbf{H} \mathbf{N M R}\left(400 \mathrm{MHz}, \mathrm{CDCl}_{3}\right) \delta 7.44(\mathrm{~d}$,
$\begin{aligned} & J=8.4 \mathrm{~Hz}, 2 \mathrm{H}), 7.34-7.30(\mathrm{~m}, 3 \mathrm{H}), 5.78(\mathrm{q}, J=6.8 \mathrm{~Hz}, 1 \mathrm{H}), \\ & 3.92(\mathrm{~d}, J=4.4 \mathrm{~Hz}, 2 \mathrm{H}), 1.63(\mathrm{~d}, J=6.8 \mathrm{~Hz}, 3 \mathrm{H}) ;{ }^{13} \mathbf{C ~ N M R}\end{aligned}$ $\left(101 \mathrm{MHz} \mathrm{CDCl}_{3}\right) \delta 167.3 .131 .8,128.8,128.3,121.9,86.3,85.4,62.5,50.3,21.4$. IR $(\mathrm{KBr}): \vee 3066,2980,2921,1741,1435,1266,1079,952,744 \mathrm{~cm}^{-1}$. HRMS (ESI) calcd for $\left[\mathrm{C}_{12} \mathrm{H}_{11} \mathrm{~N}_{3} \mathrm{O}_{2}+\mathrm{K}\right]^{+}$requires 268.0483 , found $268.0485\left[\mathrm{M}^{+}+\mathrm{K}\right]$. 

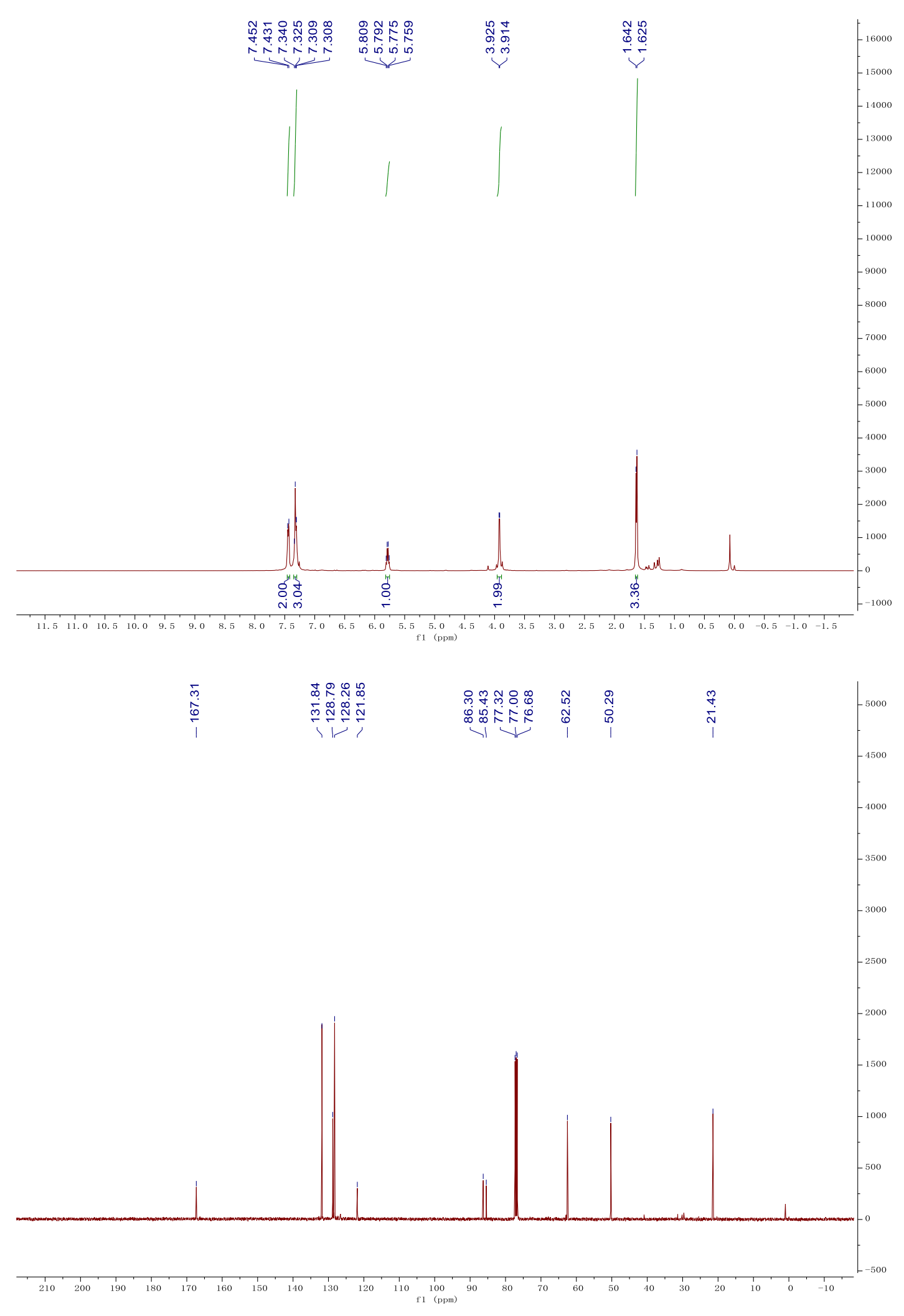


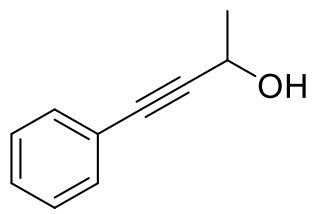

4-Phenylbut-3-yn-2-ol (5). A known compound ${ }^{[11]}$. Yield: $19 \mathrm{mg}$, 86\%. Colorless oil. ${ }^{1} \mathbf{H}$ NMR $\left(400 \mathrm{MHz}, \mathrm{CDCl}_{3}\right) \delta$ 7.44-7.41 (m, 2H), 7.31-7.29 (m, 3H), $4.76(\mathrm{q}, J=3.6 \mathrm{~Hz}, 1 \mathrm{H}), 2.13(\mathrm{~s}, 1 \mathrm{H})$, $1.55(\mathrm{~d}, J=6.4 \mathrm{~Hz}, 3 \mathrm{H}) .{ }^{13} \mathrm{C} \mathbf{N M R}\left(101 \mathrm{MHz}, \mathrm{CDCl}_{3}\right) \delta 131.8,128.3,128.2,122.5$, 90.9, 84.0, 58.8, 24.4.

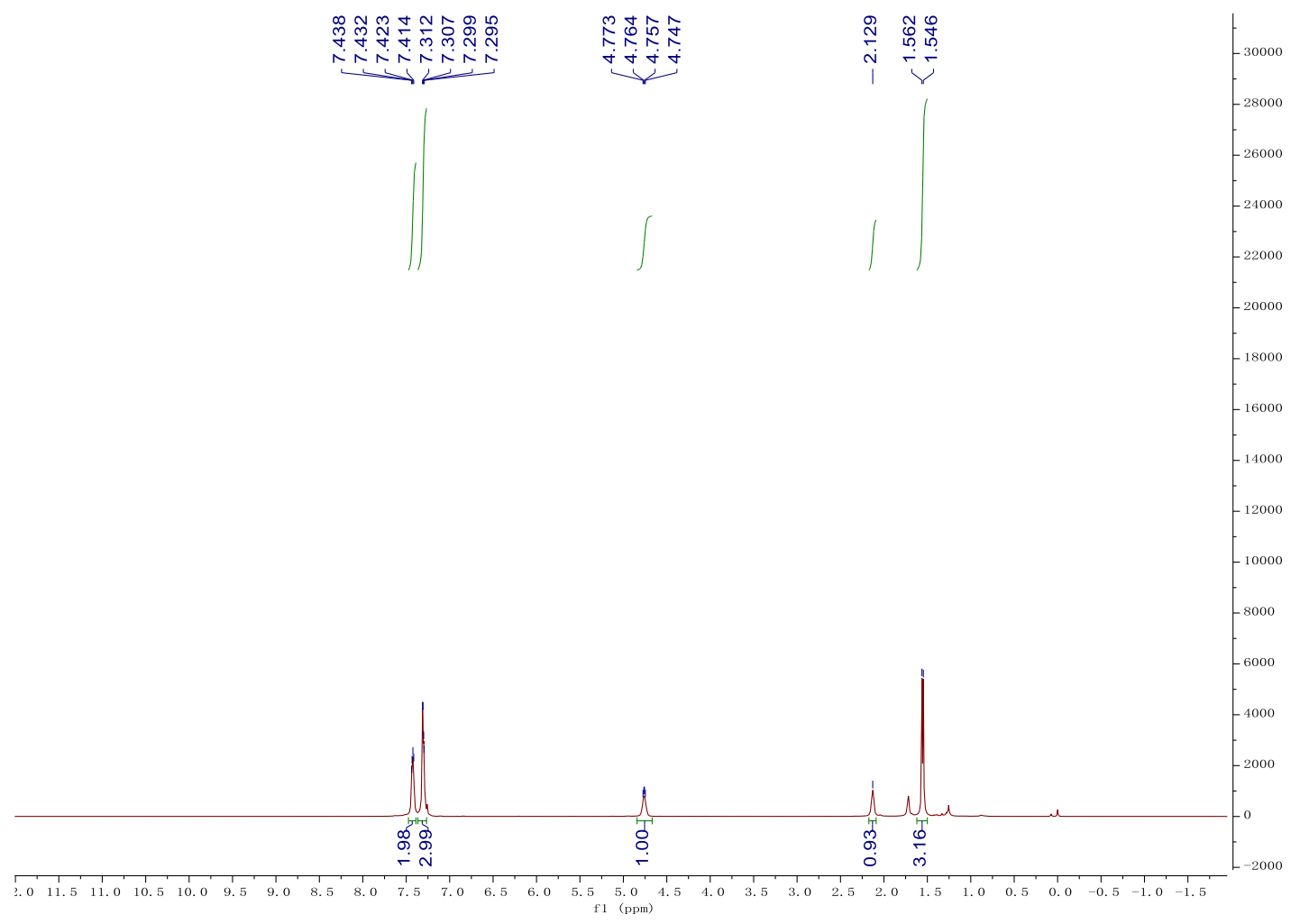




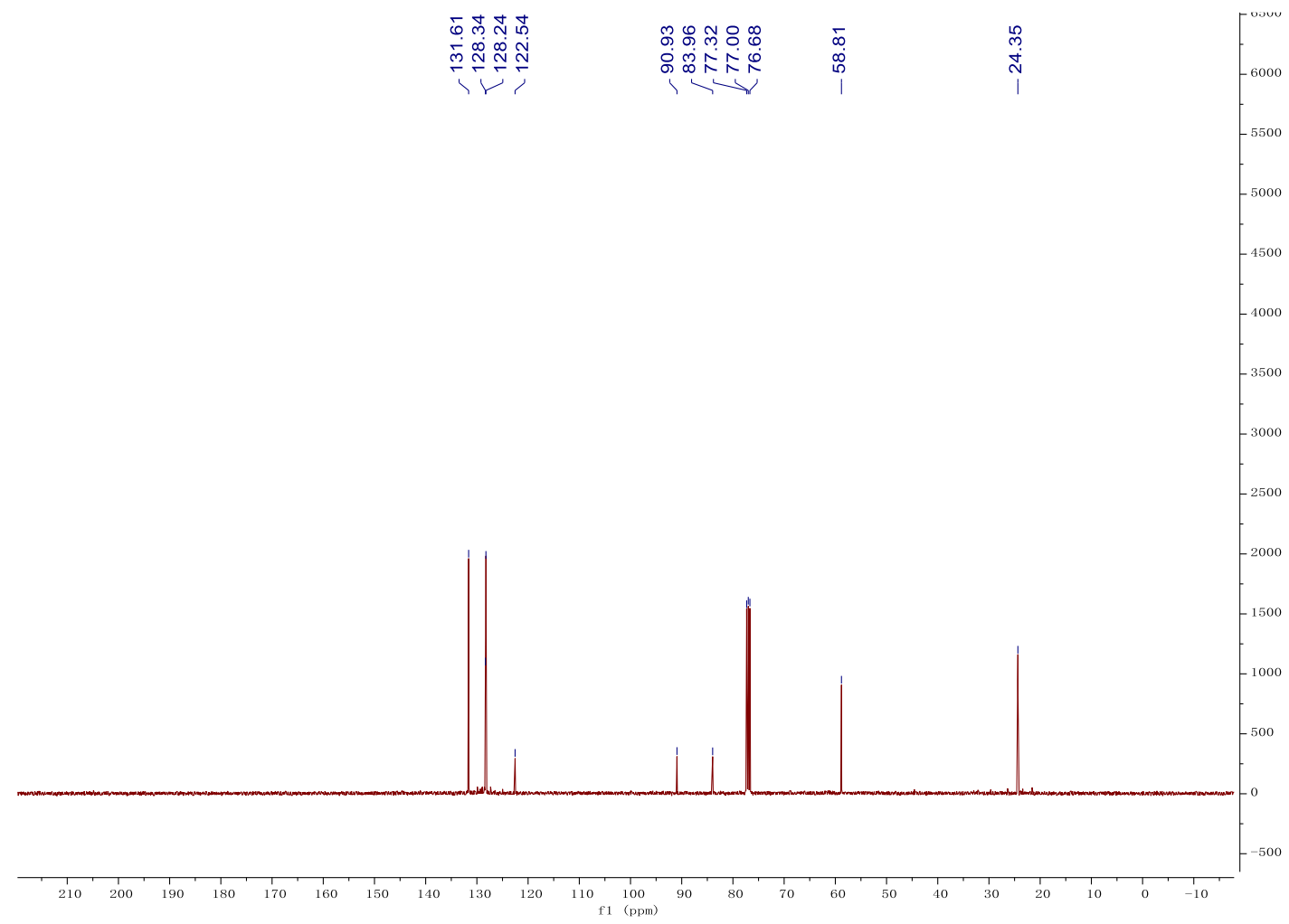

\section{REFERENCES}

(1) Takizawa, S.; Groger, H.; Sasai, H. Chem. Eur. J., 2015, 21, 8992.

(2) Tao, B.; Ruble, J. C.; Hoic, D. A.; Fu, G. C. J. Am. Chem. Soc. 1999, 121, 5091.

(3) Yang, Y-L.; Zhang, Z.; Zhang, X.-N.; Wang, D.; Wei, Y.; Shi, M. Chem.

Commun., 2014, 50, 115.

(4) Shatskiy, A.; Kivijarvi, T.; Lundberg, H.; Tinnis, F.; Adolfsson, H.

ChemCatChem., 2015, 7, 3818.

(5) Nishimoto, Y.; Okita, A.; Yasuda, M.; Baba, A. Org. Lett., 2012, 14, 1846.

(6) Sharma, B. M.; Rathod, J.; Gonnade, R. G.; Kumar, P. J Org. Chem. 2018, 83, 9353.

(7) Čikotiene, I. Org. Lett., 2014, 16, 2260.

(8) Schwier, T.; Rubin, M.; Gevorgyan, V. Org. Lett., 2004, 6, 1999.

(9) Chen, F.; Zhang, Y.; Yu, L.; Zhu, S. Angew. Chem. Int. Ed., 2017, 56, 2022.

(10) Egi, M.; Sugiyama K.; Saneto, M.; Hanada, R.; Kato, K.; Akai, S. Angew. Chem. Int. Ed., 2013, 52, 3654. 
(11) Zhang,Y.-M.; Yuan, M.-L.; Liu,W.-P.; Xie, J.-H.; Zhou, Q.-L. Org. Lett., 2018, 20, 4486 